\title{
HIGHER-DIMENSIONAL WILLMORE ENERGIES VIA MINIMAL SUBMANIFOLD ASYMPTOTICS
}

\author{
C. ROBIN GRAHAM AND NICHOLAS REICHERT
}

\begin{abstract}
A conformally invariant generalization of the Willmore energy for compact immersed submanifolds of even dimension in a Riemannian manifold is derived and studied. The energy arises as the coefficient of the log term in the renormalized area expansion of a minimal submanifold in a Poincaré-Einstein space with prescribed boundary at infinity. Its first variation is identified as the obstruction to smoothness of the minimal submanifold. The energy is explicitly identified for the case of submanifolds of dimension four. Variational properties of this four-dimensional energy are studied in detail when the background is a Euclidean space or a sphere, including identifications of critical embeddings, questions of boundedness above and below for various topologies, and second variation.
\end{abstract}

\section{INTRODUCTION}

The Willmore energy $\int_{\Sigma}|H|^{2} d a_{\Sigma}$ of a compact immersed surface $\Sigma \subset \mathbb{R}^{n}$ measures the total bending of the surface. A basic property is its conformal invariance. In this paper we derive a conformally invariant generalization of the Willmore energy for compact immersed submanifolds $\Sigma$ of even dimension $k \geq 2$ in a Riemannian manifold $(M, g)$ of dimension $n>k$. Our energy $\mathcal{E}$ is defined via an inductive algorithm which for $k>4$ is prohibitively difficult to carry out to obtain explicit formulae. However, we do derive the formula for the $k=4$ energy, which we use to study some of its basic variational properties when $(M, g)$ is a Euclidean space or a sphere.

Our energy arises upon consideration of a Plateau problem at infinity for minimal submanifolds of dimension $k+1$ of an asymptotically Poincaré-Einstein space $\left(X, g_{+}\right)$of dimension $n+1$ whose boundary at infinity is $(M, g)$. In case $(M, g)$ is a Euclidean space or a sphere, $\left(X, g_{+}\right)$is the corresponding half-space or ball model of hyperbolic space. Existence theory for minimal currents in the case that $g_{+}$is hyperbolic is discussed in [A1, A2. Here we are concerned with formal asymptotics: we search for a submanifold $Y^{k+1} \subset \bar{X}$ satisfying $Y \cap M=\Sigma$, which is minimal to high order at infinity. It turns out that the minimality condition uniquely determines the Taylor expansion of $Y$ to order $k+2$, at which point there is generically an obstruction $\mathcal{H} \in \Gamma(N \Sigma)$ to existence of a smooth $Y$. Here $N \Sigma$ denotes the normal bundle to $\Sigma$ in $M$.

The area of any such asymptotically minimal $Y$ is infinite. However, finite quantities can be obtained by consideration of an asymptotic expansion of the area. One takes $X=M \times\left(0, \epsilon_{0}\right)_{r}$ near infinity and writes the Poincaré-Einstein metric in normal form relative to $g$ as

$$
g_{+}=\frac{d r^{2}+g_{r}}{r^{2}}
$$


where $g_{r}$ is a 1-parameter family of metrics on $M$ satisfying $g_{0}=g$. The area of $Y \cap\{r>\epsilon\}$ has an asymptotic expansion in $\epsilon$, and the generalized Willmore energy $\mathcal{E}$ is defined to be the coefficient of $\log \frac{1}{\epsilon}$ in the renormalized area expansion. This turns out to be expressible as an integral over $\Sigma$ of a scalar invariant of the local submanifold geometry of $\Sigma$ in $(M, g)$. The integrand involves derivatives of the second fundamental form of order up to $(k-2) / 2$.

This area renormalization procedure was described in [GW] and has been studied in different contexts by various authors. A main focus has been the constant term in the renormalized area expansion, usually called the renormalized area. When $k$ is odd, the renormalized area is a global invariant of a minimal submanifold of a Poincaré-Einstein space and there is no $\log \frac{1}{\epsilon}$ term in the expansion. The primary interest of [GW] was the anomaly for the renormalized area when $k$ is even, measuring its failure to be conformally invariant under rescaling of $g$. The paper [GW] did point out the conformal invariance of the coefficient of the $\log \frac{1}{\epsilon}$ term and identified it as the Willmore energy when $k=2$. The contribution of the present paper, then, is to follow up with further analysis of this energy for $k>2$, particularly from the point of view of regarding it as a generalization of the $k=2$ Willmore energy.

As is well-known and described in [Gr1], there is an analogous renormalization procedure for the volume of the asymptotically Poincaré-Einstein manifold $\left(X, g_{+}\right)$itself. In this case, when $n$ is even, the coefficient of the $\log \frac{1}{\epsilon}$ term in the expansion is a conformal invariant of $(M, g)$ which equals a multiple of the integral of Branson's $Q$-curvature. A basic result ([HSS], [GH]) in this setting is that the metric variation of this coefficient is a multiple of the ambient obstruction tensor, which is a multiple of the coefficient of the first log term in the expansion of $g_{r}$. In Theorem 4.3, we prove the analogous result for the generalized Willmore energy $\mathcal{E}$ : its variational derivative with respect to variations of $\Sigma$ is the negative of the obstruction field $\mathcal{H}$. In particular, this identifies the Euler-Lagrange equation for the energy $\mathcal{E}$ as the equation $\mathcal{H}=0$. As a consequence, in Proposition 4.5 we deduce that if $\left(M^{n}, g\right)$ is Einstein and $\Sigma$ is a minimal submanifold, then $\Sigma$ is critical for $\mathcal{E}$. This generalizes a well-known property of the $k=2$ Willmore energy and produces many examples of $\mathcal{E}$-critical manifolds. This can be viewed as an analogue in this setting of the fact that the ambient obstruction tensor vanishes for Einstein metrics.

In Corollary 5.3. we identify explicitly the $k=4$ energy $\mathcal{E}$ for general background $\left(M^{n}, g\right)$ with $n \geq 5$. When $M=\mathbb{R}^{n}$ with the Euclidean metric, our energy simplifies to:

$$
\mathcal{E}=\frac{1}{128} \int_{\Sigma}\left(|\nabla H|^{2}-\left|L^{t} H\right|^{2}+\frac{7}{16}|H|^{4}\right) d a_{\Sigma} .
$$

Here $L: S^{2} T \Sigma \rightarrow N \Sigma$ is the second fundamental form and $H=\operatorname{tr} L \in \Gamma(N \Sigma)$ is the mean curvature vector. $L^{t}: N \Sigma \rightarrow S^{2} T \Sigma$ denotes the dual linear transformation and $\nabla: \Gamma(N \Sigma) \rightarrow \Gamma\left(T^{*} \Sigma \otimes N \Sigma\right)$ the normal bundle connection induced by the Levi-Civita connection of $g$. This energy was derived for $\Sigma^{4} \subset \mathbb{R}^{5}$ in Gu2 by calculating how various quantities transform under conformal motions of $\mathbb{R}^{5}$ and searching for a linear combination which is conformally invariant. However, that derivation dropped a factor of -2 in the calculations, so ended up with incorrect coefficients for $\left|L^{t} H\right|^{2}$ and $|H|^{4}$; compare (1.1) above with (61) of [Gu2]. When $M=S^{n}$ with the round metric of sectional curvature 1, our general formula reduces to:

$$
\mathcal{E}=\frac{1}{128} \int_{\Sigma}\left(|\nabla H|^{2}-\left|L^{t} H\right|^{2}+\frac{7}{16}|H|^{4}+6|H|^{2}+48\right) d a_{\Sigma}
$$


There are parallels that suggest that $\mathcal{E}$ should be regarded as the correct $k=4$ analogue of the $k=2$ Willmore energy, and that $\mathcal{E}$-critical submanifolds of $\mathbb{R}^{n}$ or $S^{n}$ are analogues of Willmore surfaces. We mentioned above that $\mathcal{E}$ shares with the $k=2$ Willmore energy the fact that minimal submanifolds of $\mathbb{R}^{n}$ or $S^{n}$ are critical. For $k=4$ this is evident from the fact that all the nonconstant terms in the integrands of (1.1) and (1.2) are at least quadratic in $H$. So, as examples of 4-dimensional $\mathcal{E}$-critical manifolds we have: totally geodesic $S^{4} \subset S^{n}$, or any round $S^{4} \subset \mathbb{R}^{n}$, or the usual minimal 4-dimensional products of spheres in $S^{n}$. The latter give rise to $\mathcal{E}$-critical "anchor rings" in Euclidean spaces via stereographic projection. In light of the interest of the Willmore conjecture, it is natural to ask about the behavior of $\mathcal{E}$ as one varies over immersions of $\Sigma^{4}$ having the topologies of these $\mathcal{E}$-critical anchor rings. We show:

Proposition 1.1. $\mathcal{E}$ is unbounded above and below over embeddings of any of the following:

$$
\begin{gathered}
S^{2} \times S^{2} \subset S^{5} \\
S^{1} \times S^{3} \subset S^{5} \\
S^{1} \times S^{1} \times S^{2} \subset S^{6} \\
S^{1} \times S^{1} \times S^{1} \times S^{1} \subset S^{7} .
\end{gathered}
$$

Proposition 1.1 rules out the most naive formulations of a 4-dimensional Willmore Conjecture for $\mathcal{E}$. As we discuss in $\$ 6.4$ (see also $\mathrm{Gu2}$ ), it is possible to modify $\mathcal{E}$ to obtain non-negative conformally invariant energies by adding appropriate multiples of the fourth power of the norm of the trace-free second fundamental form. But then one loses the property that minimal submanifolds of $\mathbb{R}^{n}$ and $S^{n}$ are critical. This seems to us an important geometric property of a higher-dimensional energy to be regarded as a true analog of the Willmore energy.

We do not know whether $\mathcal{E}$ is bounded below over immersions of $S^{4}$ in $\mathbb{R}^{5}$. It is unbounded above: we used Mathematica to calculate $\mathcal{E}$ explicitly for the family of ellipsoids in $\mathbb{R}^{5}$ with axes of length $(1,1,1,1, a)$ with $a>0$. From this one can deduce that $\mathcal{E} \rightarrow \infty$ as $a \rightarrow 0$ and as $a \rightarrow \infty$. Moreover, a numerical plot suggests that $\mathcal{E}$ is convex as a function of $a$ and has a unique minimum at $a=1$, corresponding to the round $S^{4}$. As far as we know, it is plausible that a round $S^{4} \subset \mathbb{R}^{5}$ minimzes $\mathcal{E}$ over all immersions of $S^{4}$ in $\mathbb{R}^{5}$; it would be interesting to resolve this question. Locally this is the case:

Proposition 1.2. The second variation of $\mathcal{E}$ at a round $S^{4} \subset \mathbb{R}^{5}$ is nonnegative, and is positive in directions transverse to the orbit of the conformal group.

It might be interesting to study the boundedness properties of $\mathcal{E}$ over immersions of $S^{1} \times$ $S^{1} \times S^{2}$ or $\left(S^{1}\right)^{4}$ in $S^{5}$. Likewise, to study variational properties of $\mathcal{E}$ for $\Sigma$ with other topologies, for instance, $\Sigma=\mathbb{C P}^{2}$. The Veronese embedding $\mathbb{C P}^{2} \rightarrow S^{7}$ is minimal; hence $\mathcal{E}$-critical. We would like to think, without real concrete evidence, that there should be some interesting variational problems for the energy $\mathcal{E}$, perhaps of min-max type.

Gover and Waldron have developed a program to study conformal hypersurface geometry based on the the singular Yamabe problem and tractor calculus (GoW1, GoW2, GoW3], GGHW], GoW5]). This includes the derivation of conformally invariant energies for hypersurfaces in both parities of dimension. These energies were made explicit in terms of 
underlying geometry for hypersurfaces of dimension 2 and 3. In [V], Vyatkin used tractor methods to derive an explicit conformally invariant energy for 4 dimensional hypersurfaces

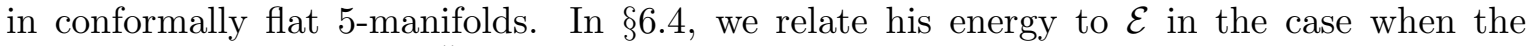
background manifold is $\mathbb{R}^{5}$. Conformally invariant hypersurface energies based on volume renormalization of singular Yamabe metrics are defined and analyzed in [Gr2, GoW4.

The paper [Z] of $Y$. Zhang was posted while this paper was in final preparation. Zhang also studies the expansion of minimal submanifolds of Poincaré-Einstein spaces through the terms of order 4 , derives a formula for $\mathcal{E}$ for $k=4$, and obtains the critical points we derive in $\$ 6.1$ for products of spheres in Euclidean spaces.

This paper is organized as follows. In $₫ 3$, we review the formal asymptotics of minimal submanifolds of Poincaré-Einstein spaces up to the order of the locally undetermined term in the expansion. The treatment in [GW] was incomplete in that it derived non-invariant asymptotic expansions in local coordinates but provided no explanation of how to formulate the results globally. Such a global formulation is needed to construct the renormalized area expansion. In Theorem 3.1 we provide an invariant formulation of the asymptotics using the normal exponential map of the boundary submanifold. All the coefficients in the expansion are invariantly defined sections of the normal bundle of $\Sigma$ determined by its geometry as a submanifold of $(M, g)$. We conclude $\oint 3$ by showing that the obstruction field $\mathcal{H} \in \Gamma(N \Sigma)$, which arises as the coefficient of the first log term in the expansion, is invariant under conformal rescalings of $g$.

In $\$ 4$, we consider the renormalized area expansion, define the energy $\mathcal{E}$, and show that it is conformally invariant. We then prove that the first variation of $\mathcal{E}$ is the negative of the obstruction field $\mathcal{H}$, and deduce that a minimal $\Sigma$ is $\mathcal{E}$-critical if $(M, g)$ is Einstein.

In 95 , we derive formulas for the expansion of the minimal submanifold and the renormalized area through order 4 . This gives formulas for $\mathcal{E}$ for $k=2,4$ and and for $\mathcal{H}$ for $k=2$. We use a formalism of Guven to identify $\mathcal{H}$ for $k=4$ when the background is a Euclidean space. These formulas for $\mathcal{H}$ are in particular the negatives of formulas for the first variation of $\mathcal{E}$ in the cases $k=2,4$. We conclude $\$ 5$ by commenting on the nonzero leading terms in the expansion coefficients, the obstruction field, and the energy.

In $₫ 6$, we analyze $\mathcal{E}$ when $\operatorname{dim} \Sigma=4$. In $₫ \underline{6.1}$ we calculate explicitly the energy of products of spheres in $S^{n}$ as a function of the radii, and use the resulting formulas to identify $\mathcal{E}$ critical embeddings of products of spheres. This gives examples of some non-minimal $\mathcal{E}$ critical embeddings. We also make a remarkable observation concerning the relationships between these energy formulas for the different topologies. In 6.2 , we stereographically project products of spheres to obtain 4-dimensional anchor rings in Euclidean spaces, thereby obtaining $\mathcal{E}$-critical anchor rings. We also analyze the energy of a non-isotropically dilated family of such anchor rings to show that $\mathcal{E}$ is unbounded above over embeddings of $S^{2} \times S^{2}$ in $S^{5}$. This combined with the results in $\$ 6.1$ enable us to prove Proposition 1.1. In $\$ 6.3$, we derive a general formula for the second variation of $\mathcal{E}$ at a minimal immersed hypersurface in $S^{5}$, precisely generalizing the corresponding formula derived in [W] for the classical Willmore energy. We apply this formula to $S^{4} \subset S^{5}$, thereby proving Proposition 1.2, and also to the standard minimal embedding $S^{2} \times S^{2} \subset S^{5}$. Finally, in 86.4 we discuss other energies obtained by modifying $\mathcal{E}$ by adding conformally invariant expressions. In particular, we construct nonnegative energies and we derive the relationship mentioned above between $\mathcal{E}$ and Vyatkin's energy. 
Acknowledgements. Research of CRG was partially supported by NSF grant \# DMS 1308266. NR held a postdoctoral position at the University of Washington while this work was carried out. His research was also supported by NSF RTG grant \# DMS 0838212 and a PIMS Postdoctoral Fellowship. He is grateful to all these institutions for their support.

\section{Notation And Conventions}

For a Riemannian manifold $\left(M^{n}, g\right)$, we denote the Levi-Civita connection by ${ }^{M} \nabla$, the curvature tensor by $R_{i j k l}$, the Ricci tensor by $\operatorname{Ric}(g)$ or $R_{i j}=R_{i k j}^{k}$, and the scalar curvature by $R=R_{i}^{i}$. Our sign convention for $R_{i j k l}$ is such that spheres have positive scalar curvature. $S^{n}(r)$ denotes the Euclidean sphere of dimension $n$ and radius $r$, and the notation $S^{n}$ is used for $S^{n}(1)$. The Schouten tensor of $(M, g)$ is

$$
P_{i j}=\frac{1}{n-2}\left(R_{i j}-\frac{R}{2(n-1)} g_{i j}\right)
$$

and the Weyl tensor is defined by the decomposition

$$
R_{i j k l}=W_{i j k l}+P_{i k} g_{j l}-P_{j k} g_{i l}-P_{i l} g_{j k}+P_{j l} g_{i k} .
$$

The Cotton and Bach tensors are

$$
C_{i j k}={ }^{M} \nabla_{k} P_{i j}-{ }^{M} \nabla_{j} P_{i k}
$$

and

$$
B_{i j}={ }^{M} \nabla^{k} C_{i j k}-P^{k l} W_{k i j l} .
$$

In invariant expressions such as these, each Latin index $i, j, k$ can be interpreted as a label for $T M$ or its dual.

$\Sigma$ will denote an immersed submanifold of $(M, g)$ of dimension $k$ via an immersion $f$ : $\Sigma \rightarrow M$. The pullback bundle $f^{*} T M$ splits as $f^{*} T M=T \Sigma \oplus N \Sigma$. We use $\alpha, \beta, \gamma$ as index labels for $T \Sigma$ and $\alpha^{\prime}, \beta^{\prime}, \gamma^{\prime}$ for $N \Sigma$. A Latin index $i$ for an element or section of $f^{*} T M$ thus corresponds to a pair $\left(\alpha, \alpha^{\prime}\right)$. So, for instance, when restricted to $\Sigma$, the Schouten tensor $P_{i j}$ splits into its tangential $P_{\alpha \beta}$, mixed $P_{\alpha \alpha^{\prime}}$, and normal $P_{\alpha^{\prime} \beta^{\prime}}$ pieces. Likewise, the restriction of the metric $g_{i j}$ to $\Sigma$ can be identified with the metric $g_{\alpha \beta}$ induced on $\Sigma$ together with the metric $g_{\alpha^{\prime} \beta^{\prime}}$ induced on $N \Sigma$. We routinely use $g_{\alpha \beta}$ and $g_{\alpha^{\prime} \beta^{\prime}}$ and their inverses to lower and raise unprimed and primed indices.

The second fundamental form is $L: S^{2} T \Sigma \rightarrow N \Sigma$, defined by $L(X, Y)=\left({ }^{M} \nabla_{X} Y\right)^{\perp}$. We typically write it as $L_{\alpha \beta}^{\alpha^{\prime}}$, or perhaps as $L_{\alpha \beta \alpha^{\prime}}$ or $L_{\alpha}{ }^{\beta} \alpha^{\prime}$ upon lowering and/or raising indices. Since $L$ has only one primed index and is symmetric in $\alpha \beta$, it is not necessary to pay attention to the order of the three indices. The mean curvature vector is $H=\operatorname{tr} L$, i.e. the section of $N \Sigma$ given by $H^{\alpha^{\prime}}=g^{\alpha \beta} L_{\alpha \beta}^{\alpha^{\prime}}=L_{\alpha}{ }^{\alpha \alpha^{\prime}}$.

The Levi-Civita connection on $M$ induces connections on $T \Sigma$ and $N \Sigma$ together with their duals and tensor products, all of which we denote $\nabla$. So, for instance, we can form the covariant derivative $\nabla_{\alpha} H^{\alpha^{\prime}}$, which is a section of $T^{*} \Sigma \otimes N \Sigma$. A point which requires some attention is that if we have a tensor on $M$ defined near $\Sigma$ (such as the Schouten tensor $P_{i j}$ ), we can form its covariant derivative ${ }^{M} \nabla_{k} P_{i j}$ and then consider on $\Sigma$ a piece of this tensor

such as ${ }^{M} \nabla_{\alpha} P_{\alpha^{\prime} \beta}$. Alternately, we can first consider on $\Sigma$ the piece $P_{\alpha^{\prime} \beta}$, which is a section of $N^{*} \Sigma \otimes T^{*} \Sigma$, and then differentiate with respect to the induced connection to obtain $\nabla_{\alpha} P_{\alpha^{\prime} \beta}$. 
Of course, these are different sections of $T^{*} \Sigma \otimes N^{*} \Sigma \otimes T^{*} \Sigma$; the distinction is indicated by the ${ }^{M}$ specifying the connection used.

Norms are always taken with respect to the metric on tensor products induced by the metric on the underlying bundle. So, for instance, in (1.1), we have

$$
\begin{gathered}
|\nabla H|^{2}=\nabla_{\alpha} H^{\alpha^{\prime}} \nabla^{\alpha} H_{\alpha^{\prime}} \\
\left|L^{t} H\right|^{2}=L_{\alpha \beta}^{\alpha^{\prime}} L_{\beta^{\prime}}^{\alpha \beta} H_{\alpha^{\prime}} H^{\beta^{\prime}} \\
|H|^{4}=\left(H_{\alpha^{\prime}} H^{\alpha^{\prime}}\right)^{2} .
\end{gathered}
$$

We often compute in local coordinates. We always use a coordinate system $\left\{\left(x^{\alpha}, u^{\alpha^{\prime}}\right)\right.$ : $\left.1 \leq \alpha \leq k, 1 \leq \alpha^{\prime} \leq n-k\right\}$ for $M$ near $\Sigma$, with the properties that $\Sigma=\left\{u^{\alpha^{\prime}}=0\right\}$ and $\partial_{\alpha} \perp \partial_{\alpha^{\prime}}$ on $\Sigma$. Hence, on $\Sigma$, the $\partial_{\alpha}$ span $T \Sigma$, the $\partial_{\alpha^{\prime}}$ span $N \Sigma$, and the mixed metric components $g_{\alpha \alpha^{\prime}}$ vanish. So our use of indices for coordinates is consistent with the abstract interpretation described above. When computing in local coordinates, partial derivatives are expressed using either of the two notations $\partial_{\alpha} u_{\beta}=u_{\beta, \alpha}$.

Our sign convention for Laplacians is that $\Delta=\sum \partial_{i}^{2}$ on Euclidean space.

When dealing with embedded submanifolds, as in 93 , we typically identify $\Sigma$ with its image and suppress mention of the immersion $f$.

\section{Formal Asymptotics}

Let $\left(M^{n},[g]\right)$ be a conformal manifold, $n \geq 2$, and $g$ a chosen metric in the conformal class. By a Poincaré metric $g_{+}$in normal form relative to $g$, we will mean a metric $g_{+}$on $X=M \times\left(0, \epsilon_{0}\right)_{r}$, for some $\epsilon_{0}>0$, of the form

$$
g_{+}=\frac{d r^{2}+g_{r}}{r^{2}}
$$

where $g_{r}$ is a smooth 1-parameter family of metrics on $M$ for which $g_{0}=g$, and satisfying

$$
\operatorname{Ric}\left(g_{+}\right)+n g_{+}=O\left(r^{n-2}\right) \text {. }
$$

These conditions uniquely determine the Taylor expansion of $g_{r} \bmod O\left(r^{n}\right)$, and it is even to this order ( $\mathrm{FG})$. The form of the expansion changes at order $n$ for solutions to higher order, but that will not be relevant here. Set $\bar{g}=r^{2} g_{+}=d r^{2}+g_{r}$. We identify $M$ with $M \times\{0\}$, and view $M=\partial X$ as the boundary at infinity relative to $g_{+}$. In case $M=\mathbb{R}^{n}$ and $g=|d x|^{2}$ is the Euclidean metric, $g_{r}=g$ is constant in $r$, and $g_{+}$is the upper-half space realization of the hyperbolic metric.

In this section we consider local geometry of embedded submanifolds of $M$. In the next section we will construct global invariants of immersed submanifolds by integration of the local invariants derived here.

Let $\Sigma \subset M$ be an embedded submanifold of dimension $k, 2 \leq k<n$, with $k$ even. We consider the formal asymptotics of embedded submanifolds $Y^{\bar{k}+1} \subset \bar{X}=M \times\left[0, \epsilon_{0}\right)$ with $\partial Y=\Sigma$ which are minimal with respect to $g_{+}$. Such a submanifold can be described invariantly in terms of a 1-parameter family of sections of the $g$-normal bundle $N \Sigma$ of $\Sigma$ in $M$ as follows.

The normal exponential map of $\Sigma$ with respect to $g$, denoted $\exp _{\Sigma}$, defines a diffeomorphism from a neighborhood of the zero section in $N \Sigma$ to a neighborhood of $\Sigma$ in $M$. 
Let $Y^{k+1} \subset M \times\left[0, \epsilon_{0}\right)$ be a smooth submanifold which is transverse to $M$ and satisfies $Y \cap M=\Sigma$. For $r \geq 0$ small, let $Y_{r} \subset M$ denote the slice of $Y$ at height $r$, defined by $Y \cap(M \times\{r\})=Y_{r} \times\{r\}$. Then $Y_{r}$ is a smooth submanifold of $M$ of dimension $k$ and $Y_{0}=\Sigma$. There is a unique section $U_{r} \in \Gamma(N \Sigma)$ so that $\exp _{\Sigma}\left\{U_{r}(p): p \in \Sigma\right\}=Y_{r}$. This defines a smooth 1-parameter family $U_{r}$ of sections of $N \Sigma$ for which

$$
Y=\left\{\left(\exp _{\Sigma} U_{r}(p), r\right): p \in \Sigma, r \geq 0\right\} .
$$

In particular, $U_{0}=0$. The submanifolds $Y \subset X$ which we consider will all be orthogonal to $M$ at $\Sigma$ with respect to $\bar{g}$. Thus the tangent bundle to $Y$ along $\Sigma$ is $T \Sigma \oplus \operatorname{span} \partial_{r}$, and the normal bundle to $Y$ along $\Sigma$ can be identified with $N \Sigma$. In this case we have $\left.\partial_{r} U_{r}\right|_{r=0}=0$, i.e. $U_{r}=O\left(r^{2}\right)$.

The condition that $Y$ is minimal becomes a system of partial differential equations on the normal vector fields $U_{r}$. Recall that minimality of $Y$ is equivalent to the statement that $H_{Y}=0$, where $H_{Y}$ denotes the mean curvature vector of $Y \subset X$ with respect to the metric $g_{+}$.

Theorem 3.1. There is a smooth $U_{r}$ so that $\left|H_{Y}\right|_{\bar{g}}=O\left(r^{k+2}\right)$. This condition uniquely determines the Taylor expansion of $U_{r}$ modulo $O\left(r^{k+2}\right)$, and this Taylor expansion is even in $r \bmod O\left(r^{k+2}\right)$. The quantity $\mathcal{H}:=\left.r^{-k-2} H_{Y}\right|_{r=0}$ defines a section of $N \Sigma$ which is independent of the choice of the $O\left(r^{k+2}\right)$ ambiguity in $U_{r}$. If nonzero, $\mathcal{H}$ is therefore an obstruction to solving $\left|H_{Y}\right|_{\bar{g}}=o\left(r^{k+2}\right)$ with $U_{r}$ a formal power series.

There is a solution to $\left|H_{Y}\left(U_{r}\right)\right|_{\bar{g}}=O\left(r^{k+3}|\log r|\right)$ of the form

$$
U_{r}=V_{r}-(k+2)^{-1} \mathcal{H} r^{k+2} \log r,
$$

where $V_{r}$ is smooth. The $r^{k+2}$ coefficient in $V_{r}$ is formally undetermined.

Remark 3.2. The same result is true for $k$ odd, but in that case $\mathcal{H}$ is always identically zero.

Remark 3.3. Boundary regularity for minimal hypersurfaces in hyperbolic space has been studied in [HL], [L1], [L2], [T], [HJ], and [HSW].

Proof. A local coordinate version of this result was derived in $\S 2$ of [GW]. We show how to reformulate Theorem 3.1 in terms of local coordinates and outline the proof, referring to GW] for some details.

We will work in geodesic normal coordinates on $M$ near $\Sigma$. Choose a local coordinate system $\left\{x^{\alpha}: 1 \leq \alpha \leq k\right\}$ for an open subset $\mathcal{V} \subset \Sigma$ and a local frame $\left\{e_{\alpha^{\prime}}(x): 1 \leq \alpha^{\prime} \leq n-k\right\}$ for $\left.N \Sigma\right|_{\mathcal{V}}$. Let $\left\{u^{\alpha^{\prime}}: 1 \leq \alpha^{\prime} \leq n-k\right\}$ denote the corresponding linear coordinates on the fibers of $\left.N \Sigma\right|_{\mathcal{V}}$. The map $\exp _{\Sigma}\left(u^{\alpha^{\prime}} e_{\alpha^{\prime}}(x)\right) \mapsto(x, u)$ defines a coordinate system $\left(x^{\alpha}, u^{\alpha^{\prime}}\right)$ in a neighborhood $\mathcal{W}$ of $\mathcal{V}$ in $M$, with respect to which $\Sigma$ is given by $u^{\alpha^{\prime}}=0$. For each $(x, u)$, the curve $t \mapsto(x, t u)$ is a geodesic for $g$ normal to $\Sigma$. In particular, in these coordinates the mixed metric components $g_{\alpha \alpha^{\prime}}$ vanish on $\mathcal{V}$. Extend the coordinates to $\mathcal{W} \times[0, \epsilon) \subset \bar{X}$ to be constant in $r$. If $U_{r}$ is a 1-parameter family of sections of $N \Sigma$ and we define $u^{\alpha^{\prime}}(x, r)$ by $U_{r}(x)=u^{\alpha^{\prime}}(x, r) e_{\alpha^{\prime}}(x)$, then the description (3.2) of $Y$ is the same as saying that in the coordinates $(x, u, r)$ on $X, Y$ is the graph $u^{\alpha^{\prime}}=u^{\alpha^{\prime}}(x, r)$.

The setting in $\mathrm{GW}$ was that $\left(x^{\alpha}, u^{\alpha^{\prime}}\right)$ is any local coordinate system on $M$ near a point of $\Sigma$ with the properties that $\Sigma=\left\{u^{\alpha^{\prime}}=0\right\}$ and $\partial_{\alpha} \perp \partial_{\alpha^{\prime}}$ on $\Sigma$, and $Y$ is described as 
the graph $u^{\alpha^{\prime}}=u^{\alpha^{\prime}}(x, r)$. So our geodesic normal coordinates constructed above and our description of $Y$ in terms of them are of this form.

Let $h$ denote the metric induced on $Y$ by $g_{+}$and set $\bar{h}=r^{2} h$, so $\bar{h}$ is the metric induced by $\bar{g}=d r^{2}+g_{r}$. Now $\left(x^{\alpha}, r\right)$ restrict to local coordinates on $Y$. In terms of these coordinates, $\bar{h}$ is given by:

$$
\begin{aligned}
& \bar{h}_{\alpha \beta}=g_{\alpha \beta}+2 g_{\alpha^{\prime}(\alpha} u^{\alpha^{\prime}}{ }_{, \beta)}+g_{\alpha^{\prime} \beta^{\prime}} u^{\alpha^{\prime}{ }_{, \alpha}} u_{{ }_{, \beta}}^{\beta^{\prime}} \\
& \bar{h}_{\alpha 0}=g_{\alpha \alpha^{\prime}} u^{\alpha^{\prime}}{ }_{, r}+g_{\alpha^{\prime} \beta^{\prime}} u^{\alpha^{\prime}}{ }_{, \alpha} u_{{ }_{, r}}^{\beta^{\prime}} \\
& \bar{h}_{00}=1+g_{\alpha^{\prime} \beta^{\prime}} u^{\alpha^{\prime}{ }_{, r}} u_{{ }_{, r}}^{\beta^{\prime}} .
\end{aligned}
$$

We use a ' 0 ' index for the $r$-direction. The components of $\bar{h}$ and the derivatives of $u$ are evaluated at $(x, r)$. We have written

$$
g_{r}=g_{\alpha \beta}(x, u, r) d x^{\alpha} d x^{\beta}+2 g_{\alpha \alpha^{\prime}}(x, u, r) d x^{\alpha} d u^{\alpha^{\prime}}+g_{\alpha^{\prime} \beta^{\prime}}(x, u, r) d u^{\alpha^{\prime}} d u^{\beta^{\prime}},
$$

and in (3.4), all $g_{i j}$ are understood to be evaluated at $(x, u(x, r), r)$.

It was shown in [GW] that for $g_{+}$of the form (3.1) and for $Y$ described as the graph $u^{\alpha^{\prime}}=u^{\alpha^{\prime}}(x, r)$, the usual minimal submanifold equation for a graph takes the form $\mathcal{M}(u)=0$, where

$$
\begin{aligned}
& \mathcal{M}(u)_{\gamma^{\prime}}=\left[r \partial_{r}-(k+1)+\frac{1}{2} r \mathcal{L}, r\right]\left[\bar{h}^{00} g_{\alpha^{\prime} \gamma^{\prime}} u^{\alpha^{\prime}}{ }_{, r}+\bar{h}^{\alpha 0}\left(g_{\alpha \gamma^{\prime}}+g_{\alpha^{\prime} \gamma^{\prime}} u^{\alpha^{\prime}}{ }_{, \alpha}\right)\right] \\
& +r\left[\partial_{\beta}+\frac{1}{2} \mathcal{L}_{, \beta}\right]\left[\bar{h}^{0 \beta} g_{\alpha^{\prime} \gamma^{\prime}} u^{\alpha^{\prime}}{ }_{, r}+\bar{h}^{\alpha \beta}\left(g_{\alpha \gamma^{\prime}}+g_{\alpha^{\prime} \gamma^{\prime}} u^{\alpha^{\prime}}{ }_{, \alpha}\right)\right] \\
& -\frac{1}{2} r \bar{h}^{\alpha \beta}\left[g_{\alpha \beta, \gamma^{\prime}}+2 g_{\alpha \alpha^{\prime}, \gamma^{\prime}} u_{, \beta}^{\alpha^{\prime}}+g_{\alpha^{\prime} \beta^{\prime}, \gamma^{\prime}} u_{, \alpha}^{\alpha^{\prime}} u_{, \beta}^{\beta^{\prime}}\right] \\
& -r \bar{h}^{\alpha 0}\left[g_{\alpha \alpha^{\prime}, \gamma^{\prime}} u^{\alpha^{\prime}{ }_{, r}}+g_{\alpha^{\prime} \beta^{\prime}, \gamma^{\prime}} u^{\alpha^{\prime}{ }_{, \alpha}} u^{\beta^{\prime}}{ }_{, r}\right] \\
& -\frac{1}{2} r \bar{h}^{00}\left[g_{\alpha^{\prime} \beta^{\prime}, \gamma^{\prime}} u^{\alpha^{\prime}}{ }_{, r} u^{\beta^{\prime}}{ }_{, r}\right] \text {. }
\end{aligned}
$$

Here $\mathcal{L}=\log (\operatorname{det} \bar{h})$. Components of $\bar{h}$ and $\mathcal{M}(u)$ are evaluated at $(x, r)$, and all $g_{i j}$ and derivatives thereof are evaluated at $(x, u(x, r), r)$. The equation $\mathcal{M}(u)=0$ is the equation we will use to study the asymptotics of $U_{r}$.

We next relate $\mathcal{M}(u)$ to the mean curvature $H_{Y}$. Recall that $-H_{Y}$ is the first variation of area of $Y$, in the sense that if $F_{t}: Y \rightarrow X$ is a compactly supported variation of $Y$, then

$$
A\left(F_{t}(Y)\right)^{\cdot}=-\int_{Y}\left\langle H_{Y}, \dot{F}\right\rangle_{g_{+}} d a_{Y}
$$

Here $A$ denotes the area and $d a_{Y}$ the area density, both with respect to $g_{+}$, and denotes $\left.\partial_{t}\right|_{t=0}$. The area $A\left(F_{t}(Y)\right)$ itself is infinite, but $A\left(F_{t}(Y)\right)^{\circ}$ is well-defined and finite since the variation is compactly supported in $X$. The usual derivation of the minimal submanifold equation (3.5) for a graph amounts to considering variations of the form $F_{t}(x, u, r)=\left(x, u_{t}, r\right)$ relative to coordinates $(x, u, r)$ as above. That derivation shows that for such variations, one has

$$
\dot{A}=-\int_{Y} r^{-1} \mathcal{M}(u)_{\gamma^{\prime}} \dot{u}^{\gamma^{\prime}} d a_{Y}
$$


Therefore

$$
r^{-1} \mathcal{M}(u)_{\gamma^{\prime}} \dot{u}^{\gamma^{\prime}}=\left\langle H_{Y}, \dot{F}\right\rangle_{g_{+}}=r^{-2}\left\langle H_{Y}, \dot{F}\right\rangle_{\bar{g}} .
$$

If we write $H_{Y}=H^{\beta} \partial_{\beta}+H^{\beta^{\prime}} \partial_{\beta^{\prime}}+H^{0} \partial_{r}$, then it follows that

$$
g_{\beta \gamma^{\prime}} H^{\beta}+g_{\beta^{\prime} \gamma^{\prime}} H^{\beta^{\prime}}=r \mathcal{M}(u)_{\gamma^{\prime}} .
$$

On the other hand, $H_{Y}$ is normal to $Y$, so

$$
\left\langle H_{Y}, \partial_{\alpha}+u^{\alpha^{\prime}},{ }_{\alpha} \partial_{\alpha^{\prime}}\right\rangle_{\bar{g}}=0, \quad\left\langle H_{Y}, \partial_{r}+u^{\alpha^{\prime}}{ }_{r} \partial_{\alpha^{\prime}}\right\rangle_{\bar{g}}=0 .
$$

These can be rewritten

$$
\left(g_{\alpha \beta}+g_{\alpha^{\prime} \beta} u^{\alpha^{\prime}}{ }_{\alpha}\right) H^{\beta}=-\left(g_{\alpha \beta^{\prime}}+g_{\alpha^{\prime} \beta^{\prime}} u^{\alpha^{\prime}}, \alpha\right) H^{\beta^{\prime}}, \quad H^{0}=-g_{\alpha^{\prime} \beta} u^{\alpha^{\prime}{ }_{r}} H^{\beta}-g_{\alpha^{\prime} \beta^{\prime}} u^{\alpha^{\prime}}{ }_{r} H^{\beta^{\prime}} .
$$

Since $g_{\alpha \beta}$ is smooth and nonsingular up to $r=0$ and $g_{\alpha^{\prime} \beta}=0$ at $r=0$, the first equation can be solved to express $H^{\beta}$ as a linear function of $H^{\beta^{\prime}}$ near $r=0$. The second equation then gives $H^{0}$ as a function of $H^{\beta^{\prime}}$ near $r=0$. Then (3.6) can be used to solve for $H^{\beta^{\prime}}$ in terms of $r \mathcal{M}(u)_{\gamma^{\prime}}$. It follows that $r|\mathcal{M}(u)|_{\bar{g}}$ and $\left|H_{Y}\right|_{\bar{g}}$ vanish to the same order at $r=0$.

As discussed in $\mathrm{GW}$, the asymptotics of $u(x, r)$ can be derived inductively from the equation $\mathcal{M}(u)=0$, starting with the initial condition $u(x, 0)=0$. For instance, upon simply setting $r=0$, the last four lines of (3.5) vanish and the first gives $u^{\alpha^{\prime}}{ }_{, r}=0$. Suppose inductively that $u$ satisfies $\mathcal{M}(u)=O\left(r^{m-1}\right)$. It is not hard to see directly from (3.5) that

$$
\mathcal{M}\left(u+w r^{m}\right)_{\gamma^{\prime}}=\mathcal{M}(u)_{\gamma^{\prime}}+m(m-k-2) g_{\gamma^{\prime} \alpha^{\prime}} w^{\alpha^{\prime}} r^{m-1}+O\left(r^{m}\right) .
$$

(The only contribution at order $m-1$ comes from the first term on the right-hand side.) So if $m<k+2$, one can uniquely determine $\left.w\right|_{r=0}$ to make $\mathcal{M}\left(u+w r^{m}\right)=O\left(r^{m}\right)$. Hence the Taylor expansion of $u \bmod O\left(r^{k+2}\right)$ is uniquely determined by the equation $|\mathcal{M}(u)|_{\bar{g}}=$ $O\left(r^{k+1}\right)$. By the discussion in the previous paragraph, this corresponds to $\left|H_{Y}\right|_{\bar{g}}=O\left(r^{k+2}\right)$. That $U_{r}$ is even follows by inspection of (3.5): the map $u \mapsto \mathcal{M}(u)$ respects parity.

To analyze what happens at order $k+2$, let $v$ be smooth and satisfy $\mathcal{M}(v)=O\left(r^{k+1}\right)$. It follows from (3.7) with $m=k+2$ that $\left.r^{-k-1} \mathcal{M}(v)\right|_{r=0}$ is independent of the choice of the order $r^{k+2}$ ambiguity in $v$, and if nonzero, is therefore an obstruction to solving $\mathcal{M}(u)=O\left(r^{k+2}\right)$ with $u$ smooth. To solve at this order it is necessary to introduce a log term. One calculates from (3.5) that

$$
\mathcal{M}\left(v+w r^{k+2} \log r\right)_{\gamma^{\prime}}=\mathcal{M}(v)_{\gamma^{\prime}}+(k+2) g_{\gamma^{\prime} \alpha^{\prime}} w^{\alpha^{\prime}} r^{k+1}+O\left(r^{k+2}|\log r|\right) .
$$

Hence $w^{\alpha^{\prime}}=-\left.(k+2)^{-1} g^{\alpha^{\prime} \gamma^{\prime}} r^{-k-1} \mathcal{M}(v)_{\gamma^{\prime}}\right|_{r=0}$ is the unique choice to make $u=v+w r^{k+2} \log r$ satisfy $\mathcal{M}(u)=O\left(r^{k+2}|\log r|\right)$.

Since $v$ and $u$ are unique $\bmod O\left(r^{k+2}\right)$ and the equation $\mathcal{M}(u)=0$ is a coordinate representation of the invariant condition $H_{Y}=0$, the corresponding 1-parameter families $V_{r}=v^{\alpha^{\prime}} e_{\alpha^{\prime}}$ and $U_{r}=u^{\alpha^{\prime}} e_{\alpha^{\prime}}$ of sections of $N \Sigma$ are globally and invariantly defined mod $O\left(r^{k+2}\right)$. The normal space to $Y$ at $r=0$ is $\operatorname{span}\left\{\partial_{\beta^{\prime}}\right\}$, and it follows from (3.6) that

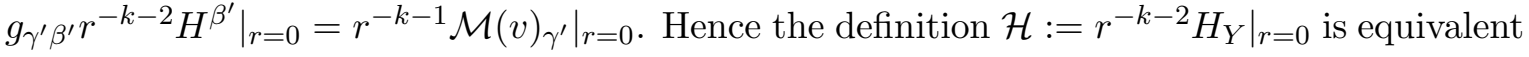
to:

$$
\mathcal{H}^{\alpha^{\prime}}=\left.g^{\alpha^{\prime} \gamma^{\prime}} r^{-k-1} \mathcal{M}(v)_{\gamma^{\prime}}\right|_{r=0} .
$$

The determination of $w$ above therefore shows that the coefficient of $r^{k+2} \log r$ in $U_{r}$ is $-(k+2)^{-1} \mathcal{H}$. 
We write the expansion of $U_{r}$ in the form

$$
U_{r}=U_{(2)} r^{2}+\ldots+U_{(k)} r^{k}-(k+2)^{-1} \mathcal{H} r^{k+2} \log r+\ldots,
$$

where each $U_{(2 j)}, 1 \leq j \leq k / 2$, is a globally, invariantly defined section of $N \Sigma$ determined by the choice of metric $g$ in the conformal class. The $U_{(2 j)}$ are not conformally invariant, but $\mathcal{H}$ is:

Proposition 3.4. If $\widehat{g}=\Omega^{2} g$ with $\Omega \in C^{\infty}(M)$, then $\widehat{\mathcal{H}}=\left(\left.\Omega\right|_{\Sigma}\right)^{-(k+2)} \mathcal{H}$.

Proof. Write $\widehat{g}_{+}=\widehat{r}^{-2}\left(d \widehat{r}^{2}+\widehat{g}_{\widehat{r}}\right)$ for the analogue of (3.1) with respect to $\widehat{g}$. Then there is a diffeomorphism $\psi$ on a neighborhood of $M$ in $M \times\left[0, \epsilon_{0}\right)$, restricting to the identity on $M \times\{0\}$, for which $\psi^{*} \widehat{g}_{+}=g_{+} \bmod O\left(r^{n-2}\right)$ and $\psi^{*} \widehat{r}=\Omega r+O\left(r^{2}\right)$ ([FG]). If $Y$ satisfies $\left|H_{Y}\right|_{\bar{g}}=O\left(r^{k+2}\right)$, then $\widehat{Y}=\psi(Y)$ satisfies $\left|H_{\widehat{Y}}\right|_{\widehat{g}}=O\left(\widehat{r}^{k+2}\right)$, where the mean curvature of $\widehat{Y}$ is taken with respect to $\widehat{g}_{+}$. Since $\psi$ restricts to the identity on $M$, it follows that

$$
\widehat{\mathcal{H}}=\psi^{*} \widehat{\mathcal{H}}=\psi^{*}\left(\left.\widehat{r}^{-k-2} H_{\widehat{Y}}\right|_{\widehat{r}=0}\right)=\left.\Omega^{-(k+2)} r^{-k-2} H_{Y}\right|_{r=0}=\left(\left.\Omega\right|_{\Sigma}\right)^{-(k+2)} \mathcal{H} .
$$

\section{ENERGY}

In this section we consider immersed submanifolds of $M$. Thus let $\Sigma$ be a manifold of even dimension $k$ and $f: \Sigma \rightarrow M$ an immersion. Relative to the metric $g$ on $M$, the pullback bundle $f^{*} T M$ splits as

$$
f^{*} T M=T \Sigma \oplus N \Sigma .
$$

$f$ is locally an embedding, so the considerations of the previous section apply. In particular, Theorem 3.1 determines a 1-parameter family of sections $U_{r} \bmod O\left(r^{k+2}\right)$ of $N \Sigma$ and an obstruction field $\mathcal{H} \in \Gamma(N \Sigma)$. In this section we set $Y=\Sigma \times\left[0, \epsilon_{0}\right)$ immersed in $\bar{X}=$ $M \times\left[0, \epsilon_{0}\right)$ via the map $\widetilde{f}: \Sigma \times\left[0, \epsilon_{0}\right) \rightarrow \bar{X}$ given by:

$$
\widetilde{f}(p, r)=\left(\exp _{\Sigma} U_{r}(p), r\right) .
$$

Consider the asymptotics of the area density $d a_{Y}$ for the metric induced by $g_{+}$. We have $d a_{Y}=\varphi d a_{\Sigma} d r$ for an invariantly defined function $\varphi$ on $\Sigma \times\left(0, \epsilon_{0}\right)$. Here $d a_{\Sigma}$ denotes the area density of $\Sigma$ with respect to the metric induced by $g$. In terms of local coordinates $\left(x^{\alpha}, u^{\alpha^{\prime}}\right)$ introduced in the proof of Theorem 3.1. we have

$$
\varphi(x, r)=\sqrt{\frac{\operatorname{det} h(x, r)}{\operatorname{det} \bar{h}_{\alpha \beta}(x, 0)}}=r^{-k-1} \sqrt{\frac{\operatorname{det} \bar{h}(x, r)}{\operatorname{det} \bar{h}_{\alpha \beta}(x, 0)}}
$$

with $\bar{h}$ given by (3.4). Since $U_{r}$ is even in $r$ to order $k+2$, it follows that the expansion of $\sqrt{\frac{\operatorname{det} \bar{h}(x, r)}{\operatorname{det} \bar{h}_{\alpha \beta}(x, 0)}}$ has only even terms through order $k$. Hence we can write

$$
d a_{Y}=r^{-k-1}\left[a^{(0)}+a^{(2)} r^{2}+\ldots+a^{(k)} r^{k}+\ldots\right] d a_{\Sigma} d r
$$

for invariantly defined functions $a^{(2 j)}, 1 \leq j \leq k / 2$, on $\Sigma$ determined by

$$
\sqrt{\frac{\operatorname{det} \bar{h}(\cdot, r)}{\operatorname{det} \bar{h}_{\alpha \beta}(\cdot, 0)}}=a^{(0)}+a^{(2)} r^{2}+\ldots+a^{(k)} r^{k}+\ldots
$$


In particular, $a^{(0)}=1$. The $a^{(2 j)}$ are called the renormalized area coefficients for $\Sigma$.

Assume now that $\Sigma$ is compact. It follows upon integration of (4.2) that for $\epsilon_{0}$ fixed,

$$
\operatorname{Area}\left(Y \cap\left\{\epsilon<r<\epsilon_{0}\right\}\right)=A_{0} \epsilon^{-k}+A_{2} \epsilon^{-k+2}+\ldots+A_{k-2} \epsilon^{-2}+\mathcal{E} \log \frac{1}{\epsilon}+O(1)
$$

as $\epsilon \rightarrow 0$, with

$$
A_{2 j}=\frac{1}{k-2 j} \int_{\Sigma} a^{(2 j)} d a_{\Sigma}, \quad 0 \leq j \leq k / 2-1, \quad \mathcal{E}=\int_{\Sigma} a^{(k)} d a_{\Sigma} .
$$

Proposition 4.1. $\mathcal{E}$ is independent of the choice of representative metric $g$.

Proof. Let $\widehat{g}$ be a conformally related metric. There is a uniquely determined defining function $\widehat{r}$ in a neighborhood of $M$ in $\bar{X}$ such that $\left.\widehat{r}^{2} g_{+}\right|_{T M}=\widehat{g}$ and $|d \widehat{r} / \widehat{r}|_{g_{+}}=1$. The difference $\mathcal{E}-\widehat{\mathcal{E}}$ is the coefficient of $\log \frac{1}{\epsilon}$ in the expansion of $\operatorname{Area}(Y \cap\{\epsilon<r\})-\operatorname{Area}(Y \cap\{\epsilon<\widehat{r}\})$. Now $\widehat{r}>\epsilon$ is equivalent to $r>\epsilon b(x, \epsilon)$ for a smooth positive function $b(x, \epsilon)$. Writing $\operatorname{Area}(Y \cap\{\epsilon<r\})-\operatorname{Area}(Y \cap\{\epsilon<\widehat{r}\})$ as an integral, it follows without difficulty that the coefficient of $\log \frac{1}{\epsilon}$ in its expansion is equal to zero. See Proposition 2.1 of [GW] for details.

The motivation for viewing $\mathcal{E}$ as a version of the Willmore energy is the fact, derived in GW], that when $k=2$ and $(M, g)$ is 3 -dimensional Euclidean space, $\mathcal{E}$ reduces to a multiple of the usual Willmore energy of $\Sigma$. This derivation will be reviewed in $\$ 5$.

Remark 4.2. There are other natural invariant immersions $\Sigma \times\left[0, \epsilon_{0}\right) \rightarrow M \times\left[0, \epsilon_{0}\right)$ having the same image as $\widetilde{f}$, which give rise to different coefficients $a^{(2 j)}$ in (4.2). The quantity Area $\left(Y \cap\left\{\epsilon<r<\epsilon_{0}\right\}\right)$ is independent of the choice of parametrization, so as long as the immersion takes the form $(p, r) \rightarrow\left(\Phi_{r}(p), r\right)$ for a 1-parameter family of immersions $\Phi_{r}: \Sigma \rightarrow M$ satisfying $\Phi_{0}=f$, the coefficients $A_{2 j}$ given by (4.5) will be the same. The corresponding $a^{(2 j)}$ will differ by a divergence. Consequently, one might not expect that the specific coefficients $a^{(2 j)}$ will play as fundamental a role as they do for the case of volume renormalization, where there is a canonical parametrization.

Next we consider the variational derivative of $\mathcal{E}$ on the space of immersions of $\Sigma$ into $M$. Let $F_{t}: \Sigma \rightarrow M, 0 \leq t<\delta$ be a variation of $\Sigma$, i.e. a smooth 1-parameter family of immersions with $F_{0}=f$. Denote by $F: \Sigma \times[0, \delta) \rightarrow M$ the map $F(p, t)=F_{t}(p)$. Let $\Sigma_{t}$ denote $\Sigma$ immersed into $M$ via $F_{t}$, let $a_{t}^{(k)}$ be the corresponding renormalized volume coefficient, and set $\mathcal{E}_{t}=\int_{\Sigma_{t}} a_{t}^{(k)} d a_{\Sigma_{t}}$. Also set $\dot{F}=\left.\partial_{t} F\right|_{t=0} \in \Gamma\left(f^{*} T M\right)$ and $\dot{\mathcal{E}}=\left.\partial_{t} \mathcal{E}_{t}\right|_{t=0}$.

Theorem 4.3. If $k \geq 2$, then

$$
\dot{\mathcal{E}}=-\int_{\Sigma}\langle\dot{F}, \mathcal{H}\rangle_{g} d a_{\Sigma}
$$

Proof. For each $t$, let $U_{r}^{t}$ be the 1-parameter family of sections of $N \Sigma_{t}$ determined by $\Sigma_{t}$ modulo $O\left(r^{k+2}\right)$ as in Theorem 3.1. For definiteness, we fix the indeterminacy in $U_{r}^{t}$ by truncating the expansion (3.9) after the log term:

$$
U_{r}^{t}(p)=U_{(2)}^{t}(p) r^{2}+\ldots+U_{(k)}^{t}(p) r^{k}-(k+2)^{-1} \mathcal{H}^{t}(p) r^{k+2} \log r, \quad p \in \Sigma .
$$


Let $\widetilde{F}_{t}: \Sigma \times\left[0, \epsilon_{0}\right) \rightarrow \bar{X}$ be the immersion defined by analogy to (4.1):

$$
\widetilde{F}_{t}(p, r)=\left(\exp _{\Sigma_{t}} U_{r}^{t}(p), r\right)
$$

and denote by $Y_{t}$ be the corresponding immersed submanifold of $\bar{X}$. Then $Y_{0}=Y$ and $U_{r}^{0}=U_{r}$.

Fix $\epsilon_{0}$ small and let $0<\epsilon<\epsilon_{0}$. Set $Y_{t}^{\epsilon}=Y_{t} \cap\left\{\epsilon<r<\epsilon_{0}\right\}$ and $Y^{\epsilon}=Y_{0}^{\epsilon}$. Then $\left.\widetilde{F}_{t}\right|_{\Sigma \times\left(\epsilon, \epsilon_{0}\right)}: \Sigma \times\left(\epsilon, \epsilon_{0}\right) \rightarrow Y_{t}^{\epsilon}$ is a variation of the manifold-with-boundary $Y^{\epsilon}$. The first variation of area formula for $\left.\widetilde{F}_{t}\right|_{\Sigma \times\left(\epsilon, \epsilon_{0}\right)}$ with background metric $g_{+}$states

$$
A\left(Y_{t}^{\epsilon}\right)^{\cdot}=-\int_{Y^{\epsilon}}\left\langle H_{Y}, \dot{\widetilde{F}}\right\rangle_{g_{+}} d a_{Y}+\left(\int_{Y \cap\left\{r=\epsilon_{0}\right\}}+\int_{Y \cap\{r=\epsilon\}}\right)\langle n, \dot{\widetilde{F}}\rangle_{g_{+}} d a_{\partial},
$$

where $d a_{\partial}$ denotes the induced area density and $n$ the outward pointing normal on $\partial Y^{\epsilon}=$ $\left(Y \cap\left\{r=\epsilon_{0}\right\}\right) \cup(Y \cap\{r=\epsilon\})$. Both sides of this equation blow up as $\epsilon \rightarrow 0$. We consider their asymptotic expansions in $\epsilon$.

According to (4.4), we have

$$
A\left(Y_{t}^{\epsilon}\right)^{\cdot}=\dot{A}_{0} \epsilon^{-k}+\dot{A}_{2} \epsilon^{-k+2}+\ldots+\dot{A}_{k-2} \epsilon^{-2}+\dot{\mathcal{E}} \log \frac{1}{\epsilon}+O(1) .
$$

So $\dot{\mathcal{E}}$ occurs as the coefficient of $\log \frac{1}{\epsilon}$ in the asymptotic expansion of the left-hand side of (4.8). The proof will be concluded by showing that the coefficient of $\log \frac{1}{\epsilon}$ on the right-hand side is $-\int_{\Sigma}\langle\dot{F}, \mathcal{H}\rangle_{g} d a_{\Sigma}$. It suffices to assume that $\dot{F}$ is supported in a small open set in $\Sigma$. In the following argument, we sometimes reduce $\epsilon_{0}$ and $\delta$ without mention.

We begin by analyzing $\dot{\widetilde{F}}$, a section of $\tilde{f}^{*} T \bar{X}$. Certainly $\left.\dot{\widetilde{F}}\right|_{\Sigma}=\dot{F}$. The decomposition $\bar{X}=M \times\left[0, \epsilon_{0}\right)$ induces a decomposition $T \bar{X}=T M \oplus T\left(\left[0, \epsilon_{0}\right)\right)$. It is clear from (4.7) that the $T\left(\left[0, \epsilon_{0}\right)\right)$-component of $\dot{\widetilde{F}}$ vanishes at each point. Choose $\mathcal{V} \subset \Sigma$ open and local coordinates $z=\left(z^{1}, \ldots, z^{n}\right)$ for $M$ in a neighborhood of $f(\mathcal{V})$. We can write

$$
\dot{\widetilde{F}}=\dot{\widetilde{F}}^{i} \partial_{z^{i}}
$$

with coefficients $\dot{\widetilde{F}}^{i}$ which are functions on $\mathcal{V} \times\left[0, \epsilon_{0}\right)$.

Lemma 4.4. Each $\dot{\widetilde{F}}^{i}$ has an asymptotic expansion of the form

$$
f_{0}+f_{2} r^{2}+\ldots+f_{k} r^{k}+f_{\log } r^{k+2} \log r+O\left(r^{k+2}\right)
$$

with coefficients $f_{2 j}, f_{\log } \in C^{\infty}(\mathcal{V})$.

Proof. As bundles on $\Sigma \times[0, \delta)$, the pullback bundle splits as $F^{*} T M=T \oplus N$, where $T_{(p, t)}=T_{p} \Sigma_{t}, N_{(p, t)}=N_{p} \Sigma_{t}$. We define exp $: N \rightarrow M$ near the zero section by $\exp v=\exp _{\Sigma_{t}} v$ for $v \in N_{(p, t)}=N_{p} \Sigma_{t}$. Let $e_{\alpha^{\prime}}(p, t), 1 \leq \alpha^{\prime} \leq n-k$, be a smooth frame for $\left.N\right|_{\mathcal{V} \times[0, \delta)}$. This frame determines a diffeomorphism $\chi: \mathcal{V} \times[0, \delta) \times \mathcal{U} \rightarrow N$ onto its image, for $\mathcal{U}$ a neighborhood of the origin in $\mathbb{R}^{n-k}$, by

$$
\chi(p, t, u)=\left(p, t, u^{\alpha^{\prime}} e_{\alpha^{\prime}}(p, t)\right) .
$$

If we represent points of $M$ using the local coordinates $z=\left(z^{1}, \ldots, z^{n}\right)$, then in these coordinates the map exp can be expressed as

$$
(\exp \propto \chi)(p, t, u)=z(p, t, u),
$$


where $z(p, t, u)$ is a smooth $\mathbb{R}^{n}$-valued function on $\mathcal{V} \times[0, \delta) \times \mathcal{U}$. In these terms, the definition (4.7) of $\widetilde{F}_{t}$ becomes

$$
\left(\widetilde{F}_{t}\right)(p, r)=\left(z\left(p, t, u_{r}(p, t)\right), r\right),
$$

where $u_{r}: \mathcal{V} \times[0, \delta) \rightarrow \mathcal{U}$ denotes the components of $U_{r}^{t}$ in the frame $e_{\alpha^{\prime}}$, defined by

$$
U_{r}^{t}(p)=u_{r}^{\alpha^{\prime}}(p, t) e_{\alpha^{\prime}}(p, t) .
$$

Now $z$ is a smooth function of $(p, t, u)$. So the asymptotic expansion in $r$ of the $z$ components of $\widetilde{F}_{t}$ can be obtained by composing the Taylor expansion of $z$ about $u=0$ with the expansion of the $u_{r}^{\alpha^{\prime}}$, which are determined by combining (4.6) with (4.11). It follows that each $z$-component of $\widetilde{F}_{t}$ has an expansion of the form (4.10) with coefficients depending smoothly on $t$. Differentiation in $t$ at $t=0$ yields the stated claim concerning $\dot{\widetilde{F}}$.

Return now to consider the right-hand side of (4.8). According to Theorem 3.1, we have $\left|H_{Y}\right|_{\bar{g}}=O\left(r^{k+3}|\log r|\right)$. Lemma 4.4 shows that $|\dot{\widetilde{F}}|_{\bar{g}}=O(1)$. Consequently $\left|\left\langle H_{Y}, \dot{\widetilde{F}}\right\rangle_{g_{+}}\right|=$ $O\left(r^{k+1}|\log r|\right)$. Since $d a_{Y}=O\left(r^{-k-1}\right) d a_{\Sigma} d r$, we deduce that

$$
\left|\int_{Y^{\epsilon}}\left\langle H_{Y}, \dot{\widetilde{F}}\right\rangle_{g_{+}} d a_{Y}\right|=O(1)
$$

as $\epsilon \rightarrow 0$. In particular, this term does not contribute to the $\log \frac{1}{\epsilon}$ term in the expansion of the right-hand side of (4.8). Likewise, the integral over $Y \cap\left\{r=\epsilon_{0}\right\}$ is independent of $\epsilon$, so does not contribute to the $\log \frac{1}{\epsilon}$ term. So the $\log \frac{1}{\epsilon}$ term in the asymptotic expansion of the right-hand side of (4.8) equals that for $\int_{Y \cap\{r=\epsilon\}}\langle n, \dot{\widetilde{F}}\rangle_{g_{+}} d a_{\epsilon}$. Here we denote the induced area density on $Y \cap\{r=\epsilon\}$ by $d a_{\epsilon}$. We regard $Y \cap\{r=\epsilon\}$ as the immersed submanifold of $X$ defined by the immersion $\widetilde{f}_{\epsilon}: \Sigma \rightarrow X, \widetilde{f}_{\epsilon}(p)=\widetilde{f}(p, \epsilon)$. We study the pointwise asymptotics in $\epsilon$ of $\left.\langle n, \dot{\widetilde{F}}\rangle_{g_{+}}\right|_{r=\epsilon}$ and of $d a_{\epsilon}$ using local coordinates.

Choose a local coordinate system $\left\{x^{\alpha}: 1 \leq \alpha \leq k\right\}$ for an open subset $\mathcal{V} \subset \Sigma$ and a local frame $\left\{e_{\alpha^{\prime}}(x): 1 \leq \alpha^{\prime} \leq n-k\right\}$ for $\left.N \Sigma\right|_{\mathcal{V}}$ as in the proof of Theorem 3.1. In the corresponding local coordinates $(x, u)$ for $M$ near $f(\mathcal{V}), Y$ is given by $u^{\alpha^{\prime}}=u^{\alpha^{\prime}}(x, r)$, where $u^{\alpha^{\prime}}(x, r)$ are the components of $U_{r}(x)$ in the frame $\left\{e_{\alpha^{\prime}}(x)\right\}$. The metric on $Y$ induced by $g_{+}$ takes the form $h=r^{-2} \bar{h}$ in the local coordinates $(x, r)$ on $\Sigma \times\left[0, \epsilon_{0}\right)$, with $\bar{h}$ given by (3.4). The outward unit conormal to $\{r=\epsilon\}$ is $-d r /|d r|_{h}$, so the outward unit normal is given in these $(x, r)$ coordinates by

$$
-\frac{1}{\sqrt{h^{00}}}\left(h^{0 \alpha} \partial_{\alpha}+h^{00} \partial_{r}\right)=-\left(\frac{h^{0 \alpha}}{\sqrt{h^{00}}} \partial_{\alpha}+\sqrt{h^{00}} \partial_{r}\right) .
$$

Thus

$$
\begin{aligned}
-n & =\widetilde{f}_{*}\left(\frac{h^{0 \alpha}}{\sqrt{h^{00}}} \partial_{\alpha}+\sqrt{h^{00}} \partial_{r}\right)=\frac{h^{0 \alpha}}{\sqrt{h^{00}}}\left(\partial_{\alpha}+u_{, \alpha}^{\alpha^{\prime}}{ }_{, \alpha} \partial_{\alpha^{\prime}}\right)+\sqrt{h^{00}}\left(\partial_{r}+u_{, r}^{\alpha^{\prime}} \partial_{\alpha^{\prime}}\right) \\
& =\frac{h^{0 \alpha}}{\sqrt{h^{00}}} \partial_{\alpha}+\left(\frac{h^{0 \gamma}}{\sqrt{h^{00}}} u_{, \gamma}^{\alpha^{\prime}}{ }_{, \gamma}+\sqrt{h^{00}} u_{{ }^{\alpha^{\prime}}}{ }_{, r}\right) \partial_{\alpha^{\prime}}+\sqrt{h^{00}} \partial_{r} .
\end{aligned}
$$

All metric coefficients $h^{0 \alpha}, h^{00}$ are evaluated at $(x, \epsilon)$. 
Take the coordinates $z$ in (4.9) to be $z=(x, u)$. Then (4.9) becomes

$$
\dot{\widetilde{F}}=\dot{\widetilde{F}}^{\alpha} \partial_{\alpha}+\dot{\widetilde{F}}^{\alpha^{\prime}} \partial_{\alpha^{\prime}}
$$

Recalling (3.1), it follows that

$$
\begin{aligned}
-\epsilon\langle n, \dot{\widetilde{F}}\rangle_{g_{+}}= & g_{\alpha \beta} \dot{\widetilde{F}}^{\alpha} \frac{\bar{h}^{0 \beta}}{\sqrt{\bar{h}^{00}}}+g_{\alpha \beta^{\prime}} \dot{\widetilde{F}}^{\alpha}\left(\frac{\bar{h}^{0 \gamma}}{\sqrt{\bar{h}^{00}}} u_{, \gamma}^{\beta^{\prime}}+\sqrt{\bar{h}^{00}} u_{, r}^{\beta^{\prime}}\right) \\
& +g_{\beta \alpha^{\prime}} \dot{\widetilde{F}}^{\alpha^{\prime}} \frac{\bar{h}^{0 \beta}}{\sqrt{\bar{h}^{00}}}+g_{\alpha^{\prime} \beta^{\prime}} \dot{\widetilde{F}}^{\alpha^{\prime}}\left(\frac{\bar{h}^{0 \gamma}}{\sqrt{\bar{h}^{00}}} u_{, \gamma}^{\beta^{\prime}}+\sqrt{\bar{h}^{00}} u_{, r}^{\beta^{\prime}}\right) .
\end{aligned}
$$

All $g_{i j}$ are evaluated at $(x, u(x, \epsilon), \epsilon)$. Likewise,

$$
d a_{\epsilon}=\sqrt{\operatorname{det} h_{\alpha \beta}(x, \epsilon)} d x=\epsilon^{-k} \sqrt{\operatorname{det} \bar{h}_{\alpha \beta}(x, \epsilon)} d x=\epsilon^{-k} \sqrt{\frac{\operatorname{det} \bar{h}_{\alpha \beta}(x, \epsilon)}{\operatorname{det} \bar{h}_{\alpha \beta}(x, 0)}} d a_{\Sigma} .
$$

Consider the asymptotic expansion in $\epsilon$ of each of the terms appearing in the right-hand sides of (4.12), (4.13). In (4.12), the factors $g_{i j}, \dot{\widetilde{F}}^{i}, \bar{h}^{0 \beta}, \sqrt{\bar{h}^{00}}, 1 / \sqrt{\bar{h}^{00}}, u^{\beta^{\prime}}{ }_{, \gamma}$ and $u^{\beta^{\prime}}{ }_{r}$ all have expansions in nonnegative powers of $\epsilon$ and positive powers of $\log \epsilon$. We analyze the powers of $\epsilon$ multiplying the $\log \epsilon$ terms in the expansions.

First consider $g_{i j}(x, u(x, \epsilon), \epsilon)$. Since $g_{i j}(x, u, r)$ is smooth, the asymptotic expansion of $g_{i j}(x, u(x, \epsilon), \epsilon)$ is obtained by composing the Taylor expansion of $g_{i j}$ in $u$ and $r$ about $u=0$, $r=0$ with the asymptotic expansion of $u$ in $r$, and then setting $r=\epsilon$. Thus it follows from (4.6) with $t=0$ that each $\log \epsilon$ term in $g_{i j}(x, u(x, \epsilon), \epsilon)$ occurs muliplied by $\epsilon$ to a power at least $k+2$. Next consider the induced metric coefficients $\bar{h}_{\alpha \beta}, \bar{h}_{\alpha 0}, \bar{h}_{00}$ given by (3.4). We claim likewise that $\log \epsilon$ occurs in each of these muliplied by $\epsilon$ to a power at least $k+2$. For $\bar{h}_{\alpha \beta}$ this is clear since the derivatives of $u^{\alpha^{\prime}}$ which appear are tangential to $\Sigma$. Now $u^{\alpha^{\prime}}{ }_{, r}$ has a term of the form $\epsilon^{k+1} \log \epsilon$. However, since $g_{\alpha \alpha^{\prime}}, u^{\alpha^{\prime}}{ }_{, \alpha}$ and $u^{\alpha^{\prime}}{ }_{, r}$ all vanish at $\epsilon=0$, the log terms in the expansions of the $u^{\alpha^{\prime}}{ }_{, r}$ occurring in $\bar{h}_{\alpha 0}$ and $\bar{h}_{00}$ all get multiplied by at least one extra factor of $\epsilon$, and the claim follows. We conclude that each $\log \epsilon$ in the inverse metric coefficients $\bar{h}^{\alpha \beta}, \bar{h}^{\alpha 0}, \bar{h}^{00}$ also is muliplied by $\epsilon$ to a power at least $k+2$. Lemma 4.4 shows that each $\log \epsilon$ in $\dot{\widetilde{F}}^{\alpha}$ and $\dot{\widetilde{F}}^{\alpha^{\prime}}$ is muliplied by $\epsilon$ to a power at least $k+2$.

The first term on the right-hand side of (4.12) is $g_{\alpha \beta} \dot{\widetilde{F}}^{\alpha} \bar{h}^{0 \beta} / \sqrt{\bar{h}^{00}}$. From the above observations it is clear that each $\log \epsilon$ term in its asymptotic expansion is muliplied by $\epsilon$ to a power at least $k+2$. Likewise for the third term $g_{\beta \alpha^{\prime}} \dot{\widetilde{F}}^{\alpha^{\prime}} \bar{h}^{0 \beta} / \sqrt{\overline{h^{00}}}$. The second and fourth terms of (4.12) include a factor $u^{\beta^{\prime}}{ }_{, r}$, whose expansion has a term of the form $\epsilon^{k+1} \log \epsilon$. Now the second term has a leading factor $g_{\alpha \beta^{\prime}}$, which vanishes at $\epsilon=0$. So each $\log \epsilon$ term in the asymptotic expansion of the second term is muliplied by $\epsilon$ to a power at least $k+2$. However, this is not the case for the fourth term. According to (4.6), $u^{\beta^{\prime}{ }_{, r}}$ has a term $-\mathcal{H}^{\beta^{\prime}} \epsilon^{k+1} \log \epsilon$. Since $\left.\dot{\widetilde{F}}\right|_{\Sigma}=\dot{F}$ and $\left.\bar{h}^{00}\right|_{\Sigma}=1$, it follows that the expansion of the fourth term of (4.12) has a term $-g_{\alpha^{\prime} \beta^{\prime}} \dot{F}^{\alpha^{\prime}} \mathcal{H}^{\beta^{\prime}} \epsilon^{k+1} \log \epsilon$. Putting all this together, we conclude that the expansion of $\langle n, \dot{\widetilde{F}}\rangle_{g_{+}}$has a term $\langle\dot{F}, \mathcal{H}\rangle_{g} \epsilon^{k} \log \epsilon$, and all other $\log \epsilon$ terms appear with a coefficient of $\epsilon^{k+1}$ or higher. 
It is clear that the factor $\sqrt{\operatorname{det} \bar{h}_{\alpha \beta}(x, \epsilon) / \operatorname{det} \bar{h}_{\alpha \beta}(x, 0)}$ in (4.13) has an expansion with a leading term of 1 and with all $\log \epsilon$ terms multiplied by a power of $\epsilon$ at least $k+2$. Combining with the conclusion of the above paragraph, it follows that the $\log \epsilon$ coefficient in the expansion of $\langle n, \dot{\widetilde{F}}\rangle_{g_{+}} d a_{\epsilon}$ is $\langle\dot{F}, \mathcal{H}\rangle_{g} d a_{\Sigma}$. Integrating over $\Sigma$ concludes the proof of Theorem 4.3 ,

As a consequence of Theorem 4.3, we deduce the following proposition.

Proposition 4.5. Let $k \geq 2$ be even and suppose $\left(M^{n}, g\right)$ is Einstein. If $\Sigma^{k}$ is a minimal immersed submanifold of $(M, g)$, then $\Sigma$ is critical for $\mathcal{E}$.

Proof. The Poincaré metric for an Einstein metric can be written explicitly: if $\operatorname{Ric}(g)=$ $2 \lambda(n-1) g$, then $g_{r}=\left(1-\lambda r^{2} / 2\right)^{2} g$ (see [FG]). So

$$
g_{+}=r^{-2}\left(d r^{2}+\left(1-\lambda r^{2} / 2\right)^{2} g\right)=d s^{2}+\left(e^{s}-\lambda e^{-s} / 2\right)^{2} g, \quad s=-\log r .
$$

It is easy to verify the general fact that if $\Sigma$ is a minimal submanifold of a Riemannian manifold $(M, g)$, then $\Sigma \times \mathbb{R}$ is a minimal submanifold of $M \times \mathbb{R}$ with respect to any warped product metric of the form $g_{+}=d s^{2}+A(s) g$, where $s$ denotes the variable in $\mathbb{R}$ and $A(s)$ is a positive function. Thus when $g$ is Einstein and $\Sigma$ is minimal, the minimal extension $Y$ in Theorem 3.1 is simply $Y=\Sigma \times \mathbb{R}$. That is, the corresponding normal field is $U_{r}=0$ $\bmod O\left(r^{k+2}\right)$. (An alternate, equivalent way to see this is simply to note that if $g_{r}=B(r) g$ for some positive function $B(r)$ and $\Sigma$ is minimal, then $u=0$ solves $\mathcal{M}(u)=0$ exactly, where recall $\mathcal{M}(u)$ is given by (3.5) $)$.

Since $U_{r}=0$ has no log term in its expansion, it must be that $\mathcal{H}=0$. By Theorem 4.3 , it follows that $\Sigma$ is critical for $\mathcal{E}$.

Proposition 4.5 implies in particular that minimal submanifolds of $\mathbb{R}^{n}$ or $S^{n}$ are critical for $\mathcal{E}$.

\section{Derivation of Formulas}

In this section we derive formulas for the renormalized area coefficients $a^{(2)}, a^{(4)}$ in the expansion (4.3), and for the coefficients $U_{(2)}, U_{(4)}$ in the expansion (3.9) of $U_{r}$. This gives formulas for the energy $\mathcal{E}$ for $k=2,4$ by integration, and for the obstruction $\mathcal{H}$ for $k=2$. We also use Theorem 4.3 and a formalism of Guven to identify $\mathcal{H}$ for $k=4$ when $(M, g)$ is a Euclidean space.

The coefficients $U_{(2 j)}$ are determined by solving the equation $\mathcal{M}(u)=0$ inductively order by order, where $\mathcal{M}(u)$ is given by (3.5). Using the fact that $u^{\alpha^{\prime}}{ }_{, r}=0$ at $r=0$, one sees easily that all terms on the right-hand side of (3.5) are $O\left(r^{2}\right)$ except for the first and third. Thus

$$
\left(r \partial_{r}-(k+1)\right)\left(\bar{h}^{00} g_{\alpha^{\prime} \gamma^{\prime}} u_{, r}^{\alpha^{\prime}}\right)-\frac{1}{2} r \bar{h}^{\alpha \beta} g_{\alpha \beta \cdot \gamma^{\prime}}=O\left(r^{2}\right) .
$$

Applying $\left.\partial_{r}\right|_{r=0}$ and raising an index gives $k u_{, r r}^{\alpha^{\prime}}=-\frac{1}{2} g^{\alpha^{\prime} \gamma^{\prime}} g^{\alpha \beta} g_{\alpha \beta, \gamma^{\prime}}$ at $r=0$. Therefore

$$
U_{(2)}=\frac{1}{2 k} H \text {. }
$$

We next turn to the identification of $a^{(2)}$ and $a^{(4)}$. We will return later to the determination of $U_{(4)}$ by further differentiation of (3.5). 
Proposition 5.1. If $k \geq 1$, then

$$
\begin{aligned}
a^{(2)=-1} & \left(\frac{k-1}{k^{2}}|H|^{2}+P^{\alpha}{ }_{\alpha}\right) \\
a^{(4)}=\frac{1}{8 k^{2}} & \left(|\nabla H|^{2}-L_{\alpha \beta}^{\alpha^{\prime}} L_{\beta^{\prime}}^{\alpha \beta} H_{\alpha^{\prime}} H^{\beta^{\prime}}+\frac{k^{2}-2 k-1}{k^{2}}|H|^{4}\right. \\
& -W^{\alpha}{ }_{\alpha^{\prime} \alpha \beta^{\prime}} H^{\alpha^{\prime}} H^{\beta^{\prime}}-2 k g^{\alpha \beta M} \nabla_{\alpha^{\prime}} P_{\alpha \beta} H^{\alpha^{\prime}}-4 k P^{\alpha}{ }_{\alpha^{\prime}} \nabla_{\alpha} H^{\alpha^{\prime}} \\
& \left.+(2 k-3) P_{\alpha}^{\alpha}{ }_{\alpha}|H|^{2}-(k+4) P_{\alpha^{\prime} \beta^{\prime}} H^{\alpha^{\prime}} H^{\beta^{\prime}}\right) \\
+ & \frac{1}{8}\left(-P^{\alpha \beta} P_{\alpha \beta}+P^{\alpha \alpha^{\prime}} P_{\alpha \alpha^{\prime}}+\left(P^{\alpha}{ }_{\alpha}\right)^{2}-\frac{1}{n-4} B^{\alpha}{ }_{\alpha}\right) \\
+ & \frac{4-k}{k} H_{\alpha^{\prime}} U_{(4)}^{\alpha^{\prime}}
\end{aligned}
$$

In the last line of the expression for $a^{(4)}$, one should substitute the formula for $U_{(4)}$ given in Proposition 5.5 and combine like terms. However, we are primarily interested in $a^{(4)}$ for $k=4$, when it is the integrand for $\mathcal{E}$. When $k=4$, the $U_{(4)}$ term does not appear. Consequently we have left the expression in the above form.

Proposition 5.1 will be proved by calculating in special coordinates. Recall the geodesic normal coordinate systems $(x, u)$ on $M$ near $\Sigma$ constructed at the beginning of the proof of Theorem 3.1 which are associated to a choice of local coordinates $x^{\alpha}$ for $\Sigma$ and a choice of frame $e_{\alpha^{\prime}}$ for $N \Sigma$. Given $p \in \Sigma$, choose the $x^{\alpha}$ so that $g_{\alpha \beta, \gamma}(p)=0$ for $1 \leq \alpha, \beta, \gamma \leq k$. (For instance, take $x^{\alpha}$ to be geodesic normal coordinates at $p$ for the induced metric on $\Sigma$.) Choose the frame $e_{\alpha^{\prime}}$ so that $\nabla_{\alpha} e_{\alpha^{\prime}}(p)=0$ for $1 \leq \alpha \leq k, 1 \leq \alpha^{\prime} \leq n-k$, where $\nabla_{\alpha}$ denotes the normal bundle connection on $N \Sigma$.

Lemma 5.2. In such coordinates $(x, u)$, the following all vanish at $p$ :

$$
g_{\alpha \beta, \gamma} \quad g_{\alpha \alpha^{\prime}, \beta} \quad g_{\alpha^{\prime} \beta^{\prime}, \alpha} \quad g_{\alpha \alpha^{\prime}, \beta^{\prime}} \quad g_{\alpha^{\prime} \beta^{\prime}, \gamma^{\prime}} .
$$

At $p$ we also have

$$
g_{\alpha \beta, \alpha^{\prime}}=-2 L_{\alpha \beta \alpha^{\prime}}
$$

and

$$
g_{\alpha \beta, \alpha^{\prime} \beta^{\prime}}=2 R_{\alpha^{\prime}(\alpha \beta) \beta^{\prime}}+L_{\alpha \gamma \beta^{\prime}} L_{\beta}{ }^{\alpha^{\prime}}+L_{\beta \gamma \beta^{\prime}} L_{\alpha}^{\gamma}{ }_{\alpha^{\prime}} .
$$

If $v$ is a section of $N \Sigma$, then at $p$ we have

$$
\nabla_{\alpha} \nabla_{\beta} v^{\alpha^{\prime}}=\left(v_{, \beta \alpha}^{\alpha^{\prime}}+g^{\alpha^{\prime} \gamma^{\prime}} g_{\beta \gamma^{\prime}, \beta^{\prime} \alpha} v^{\beta^{\prime}}\right)
$$

Proof. The $x$ coordinates were chosen so that $g_{\alpha \beta, \gamma}=0$ at $p$. We have $g_{\alpha \alpha^{\prime}, \beta}=0$ on all of $\Sigma$ since $g_{\alpha \alpha^{\prime}}=0$ on $\Sigma$ and $\partial_{\beta}$ acts tangentially. Recall from the construction of $(x, u)$ that each curve $t \mapsto(x, t u)$ is a geodesic. This implies in particular that $\Gamma_{\alpha^{\prime} \beta^{\prime}}^{\gamma}=0$ and $\Gamma_{\alpha^{\prime} \beta^{\prime}}^{\gamma^{\prime}}=0$ on $\Sigma$. The latter equation is equivalent to $g_{\alpha^{\prime} \beta^{\prime}, \gamma^{\prime}}=0$ on $\Sigma$. The former is equivalent to

$$
g_{\alpha^{\prime} \beta^{\prime}, \alpha}=g_{\alpha \alpha^{\prime}, \beta^{\prime}}+g_{\alpha \beta^{\prime}, \alpha^{\prime}} \quad \text { on } \Sigma \text {. }
$$


Since $e_{\alpha^{\prime}}=\partial_{\alpha^{\prime}}$ on $\Sigma$, the equation $\nabla_{\alpha} e_{\alpha^{\prime}}(p)=0$ is equivalent to $\Gamma_{\alpha \alpha^{\prime}}^{\beta^{\prime}}(p)=0$, which is equivalent to $g_{\alpha^{\prime} \beta^{\prime}, \alpha}=g_{\alpha \alpha^{\prime}, \beta^{\prime}}-g_{\alpha \beta^{\prime}, \alpha^{\prime}}$ at $p$. The left-hand side is symmetric in $\alpha^{\prime} \beta^{\prime}$ and the right-hand side is skew, so both must vanish. Combining with (5.5), we conclude that in fact $g_{\alpha^{\prime} \beta^{\prime}, \alpha}=g_{\alpha \alpha^{\prime}, \beta^{\prime}}=0$ at $p$.

Equation (5.2) holds on all of $\Sigma$ in any coordinates $(x, u)$ for which $\Sigma=\{u=0\}$ and $\partial_{\alpha} \perp \partial_{\alpha^{\prime}}$ on $\Sigma$. In fact, in this case $\nabla_{\partial_{\alpha}} \partial_{\beta}=\Gamma_{\alpha \beta}^{\gamma} \partial_{\gamma}+\Gamma_{\alpha \beta}^{\gamma^{\prime}} \partial_{\gamma^{\prime}}$, so $L_{\alpha \beta}^{\gamma^{\prime}}=\Gamma_{\alpha \beta}^{\gamma^{\prime}}=-\frac{1}{2} g^{\gamma^{\prime} \alpha^{\prime}} g_{\alpha \beta, \alpha^{\prime}}$.

For (5.3), the curvature tensor is given in local coordinates by

$$
-2 R_{i j k l}=g_{i k, j l}+g_{j l, i k}-g_{i l, j k}-g_{j k, i l}-2 g^{p q}\left(\Gamma_{i l p} \Gamma_{j k q}-\Gamma_{i k p} \Gamma_{j l q}\right) .
$$

The Christoffel symbols all vanish at $p$ except for

$$
\Gamma_{\alpha \beta \alpha^{\prime}}=L_{\alpha \beta \alpha^{\prime}}, \quad \Gamma_{\alpha \alpha^{\prime} \beta}=\Gamma_{\alpha^{\prime} \alpha \beta}=-L_{\alpha \beta \alpha^{\prime}} .
$$

So specializing the indices and evaluating at $p$ gives

$$
-2 R_{\alpha \alpha^{\prime} \beta \beta^{\prime}}=g_{\alpha \beta, \alpha^{\prime} \beta^{\prime}}+g_{\alpha^{\prime} \beta^{\prime}, \alpha \beta}-g_{\alpha \beta^{\prime}, \beta \alpha^{\prime}}-g_{\beta \alpha^{\prime}, \alpha \beta^{\prime}}-2 g^{\gamma \delta} L_{\alpha \gamma \beta^{\prime}} L_{\beta \delta \alpha^{\prime}} .
$$

Symmetrizing in $\alpha \beta$ gives

$$
2 R_{\alpha^{\prime}(\alpha \beta) \beta^{\prime}}=g_{\alpha \beta, \alpha^{\prime} \beta^{\prime}}+\operatorname{Sym}_{\alpha \beta}\left[\left(g_{\alpha^{\prime} \beta^{\prime}, \beta}-g_{\beta \beta^{\prime}, \alpha^{\prime}}-g_{\beta \alpha^{\prime}, \beta^{\prime}}\right), \alpha\right]-L_{\alpha \gamma \beta^{\prime}} L_{\beta}{ }^{\gamma} \alpha^{\prime}-L_{\beta \gamma \beta^{\prime}} L_{\alpha}{ }^{\gamma} \alpha^{\prime} .
$$

But

$$
\left(g_{\alpha^{\prime} \beta^{\prime}, \beta}-g_{\beta \beta^{\prime}, \alpha^{\prime}}-g_{\beta \alpha^{\prime}, \beta^{\prime}}\right)_{, \alpha}=0 \quad \text { on } \Sigma
$$

since $g_{\alpha^{\prime} \beta^{\prime}, \beta}-g_{\beta \beta^{\prime}, \alpha^{\prime}}-g_{\beta \alpha^{\prime}, \beta^{\prime}}=0$ on $\Sigma$ by (5.5), and $\partial_{\alpha}$ acts tangentially. Thus (5.3) holds.

For (5.4), write $\nabla_{\beta} v^{\alpha^{\prime}}=v^{\alpha^{\prime}}{ }_{, \beta}+\Gamma_{\beta \beta^{\prime}}^{\alpha^{\prime}} v^{\beta^{\prime}}$, apply $\nabla_{\alpha}$, and expand the right-hand side again in terms of partial derivatives and Christoffel symbols. Using the fact that all Christoffel symbols vanish at $p$ except for (5.6), one obtains at $p$ :

$$
\nabla_{\alpha} \nabla_{\beta} v^{\alpha^{\prime}}=v_{, \beta \alpha}^{\alpha^{\prime}}+\Gamma_{\beta \beta^{\prime}, \alpha}^{\alpha^{\prime}} v^{\beta^{\prime}}=v_{, \beta \alpha}^{\alpha^{\prime}}+\frac{1}{2} g^{\alpha^{\prime} \gamma^{\prime}}\left(g_{\gamma^{\prime} \beta^{\prime}, \beta}+g_{\beta \gamma^{\prime}, \beta^{\prime}}-g_{\beta \beta^{\prime}, \gamma^{\prime}}\right)_{, \alpha} v^{\beta^{\prime}} .
$$

But (5.7) gives $g_{\gamma^{\prime} \beta^{\prime}, \beta \alpha}=\left(g_{\beta \beta^{\prime}, \gamma^{\prime}}+g_{\beta \gamma^{\prime}, \beta^{\prime}}\right)_{, \alpha}$ on $\Sigma$, so substituting yields (5.4).

Proof of Proposition 5.1. Since the definition (4.2) of the $a^{(2 j)}$ and the formulae in Proposition 5.1 are coordinate-invariant, it suffices to prove them at $p$ in the coordinates $(x, u)$ constructed above. Choose the $x$ coordinates on $\Sigma$ so that $x(p)=0$. Then $p$ is represented by $(0,0,0)$ in the coordinates $(x, u, r)$ on $X$.

The induced metric components are given by (3.4), and according to (4.3), we need to calculate the Taylor expansion of $\sqrt{\operatorname{det} \bar{h}(x, r)}$. Now $g_{i j}$ is given $([\mathrm{FG}])$ by

$$
g_{i j}(x, u, r)=g_{i j}(x, u, 0)-P_{i j}(x, u, 0) r^{2}+\widetilde{B}_{i j}(x, u, 0) r^{4}+\ldots,
$$

where

$$
\widetilde{B}_{i j}=\frac{1}{4}\left[\frac{B_{i j}}{4-n}+P_{i}^{k} P_{k j}\right]
$$

By (5.1), we have

$$
u^{\alpha^{\prime}}(x, r)=\frac{1}{2 k} H^{\alpha^{\prime}}(x) r^{2}+u_{(4)}^{\alpha^{\prime}}(x) r^{4}+\ldots
$$


All $g_{i j}$ in (3.4) are evaluated at $(x, u(x, r), r)$. Since $g_{\alpha \alpha^{\prime}}(x, 0,0)=0$, it follows from (5.8) and (5.10) that $g_{\alpha \alpha^{\prime}}(x, u(x, r), r)=O\left(r^{2}\right)$. Then (3.4) shows that $\bar{h}_{\alpha 0}=O\left(r^{3}\right)$. Thus

$$
\operatorname{det} \bar{h}=\left(\operatorname{det} \bar{h}_{\alpha \beta}\right) \cdot \bar{h}_{00}+O\left(r^{6}\right) .
$$

Staring at (3.4) and recalling that $u=O\left(r^{2}\right)$, it is clear that in order to evaluate $\bar{h}_{\alpha \beta}$ and $\bar{h}_{00}$ through order $r^{4}$, we need to know $g_{\alpha \beta}$ through order $r^{4}$ and $g_{\alpha \alpha^{\prime}}$ and $g_{\alpha^{\prime} \beta^{\prime}}$ through order $r^{2}$. Taking $x=0$, corresponding to the point $p$, we have

$$
\begin{aligned}
g_{\alpha^{\prime} \beta^{\prime}}(0, u(0, r), r) & =g_{\alpha^{\prime} \beta^{\prime}}(0, u(0, r), 0)-P_{\alpha^{\prime} \beta^{\prime}} r^{2}+o\left(r^{2}\right) \\
& =g_{\alpha^{\prime} \beta^{\prime}}(0,0,0)+g_{\alpha^{\prime} \beta^{\prime}, \gamma^{\prime}}(0,0,0) u^{\gamma^{\prime}}(0, r)-P_{\alpha^{\prime} \beta^{\prime}} r^{2}+o\left(r^{2}\right) \\
& =g_{\alpha^{\prime} \beta^{\prime}}(0,0,0)-P_{\alpha^{\prime} \beta^{\prime}} r^{2}+o\left(r^{2}\right),
\end{aligned}
$$

where we used $g_{\alpha^{\prime} \beta^{\prime}, \gamma^{\prime}}(0,0,0)=0$ from Lemma 5.2. Likewise

$$
g_{\alpha \alpha^{\prime}}(0, u(0, r), r)=-P_{\alpha \alpha^{\prime}} r^{2}+o\left(r^{2}\right) .
$$

Now (5.8) gives

$$
g_{\alpha \beta}(0, u(0, r), r)=g_{\alpha \beta}(0, u(0, r), 0)-P_{\alpha \beta}(0, u(0, r), 0) r^{2}+\widetilde{B}_{\alpha \beta} r^{4}+o\left(r^{4}\right) .
$$

Expanding the first term and substituting from Lemma 5.2 and (5.10) gives

$$
\begin{aligned}
g_{\alpha \beta}(0, u(0, r), 0)= & g_{\alpha \beta}(0,0,0)+g_{\alpha \beta, \alpha^{\prime}}(0,0,0) u^{\alpha^{\prime}}(0, r)+\frac{1}{2} g_{\alpha \beta, \alpha^{\prime} \beta^{\prime}}(0,0,0) u^{\alpha^{\prime}} u^{\beta^{\prime}}+o\left(r^{4}\right) \\
= & g_{\alpha \beta}(0,0,0)-2 L_{\alpha \beta \alpha^{\prime}}\left(\frac{1}{2 k} H^{\alpha^{\prime}} r^{2}+u_{(4)}^{\alpha^{\prime}} r^{4}\right) \\
& \quad+\frac{1}{4 k^{2}}\left(R_{\alpha^{\prime} \alpha \beta \beta^{\prime}}+L_{\alpha \gamma \beta^{\prime}} L_{\beta}{ }^{\gamma}{ }_{\alpha^{\prime}}\right) H^{\alpha^{\prime}} H^{\beta^{\prime}} r^{4}+o\left(r^{4}\right) \\
= & g_{\alpha \beta}(0,0,0)-\frac{1}{k} L_{\alpha \beta \alpha^{\prime}} H^{\alpha^{\prime}} r^{2} \\
& \quad+\left(-2 L_{\alpha \beta \alpha^{\prime}} u_{(4)}^{\alpha^{\prime}}+\frac{1}{4 k^{2}}\left(R_{\alpha^{\prime} \alpha \beta \beta^{\prime}}+L_{\alpha \gamma \beta^{\prime}} L_{\beta}{ }_{\alpha^{\prime}}\right) H^{\alpha^{\prime}} H^{\beta^{\prime}}\right) r^{4}+o\left(r^{4}\right) .
\end{aligned}
$$

For use in the second term in (5.13), we have

$$
P_{\alpha \beta}(0, u(0, r), 0)=P_{\alpha \beta}(0,0,0)+P_{\alpha \beta, \alpha^{\prime}} u^{\alpha^{\prime}}+o\left(r^{2}\right)=P_{\alpha \beta}(0,0,0)+\frac{1}{2 k} P_{\alpha \beta, \alpha^{\prime}} H^{\alpha^{\prime}} r^{2}+o\left(r^{2}\right) .
$$

Substituting these into (5.13) gives

$$
\begin{aligned}
& g_{\alpha \beta}(0, u(0, r), r)=g_{\alpha \beta}(0,0,0)+\left(-\frac{1}{k} L_{\alpha \beta \alpha^{\prime}} H^{\alpha^{\prime}}-P_{\alpha \beta}(0,0,0)\right) r^{2} \\
& \quad+\left(-2 L_{\alpha \beta \alpha^{\prime}} u_{(4)}^{\alpha^{\prime}}+\frac{1}{4 k^{2}}\left(R_{\alpha^{\prime} \alpha \beta \beta^{\prime}}+L_{\alpha \gamma \beta^{\prime}} L_{\beta^{\gamma} \alpha^{\prime}}\right) H^{\alpha^{\prime}} H^{\beta^{\prime}}-\frac{1}{2 k} P_{\alpha \beta, \alpha^{\prime}} H^{\alpha^{\prime}}+\widetilde{B}_{\alpha \beta}\right) r^{4}+o\left(r^{4}\right) .
\end{aligned}
$$

Now substitute all these into (3.4). Henceforth, all $g_{i j}$ and $P_{i j}$ are understood to be evaluated at $p$. One obtains

$$
\begin{aligned}
& \bar{h}_{\alpha \beta}=g_{\alpha \beta}+D_{\alpha \beta} r^{2}+Q_{\alpha \beta} r^{4}+o\left(r^{4}\right) \\
& \bar{h}_{00}=1+E r^{2}+F r^{4}+o\left(r^{4}\right)
\end{aligned}
$$


with

$$
\begin{aligned}
D_{\alpha \beta}= & -\frac{1}{k} L_{\alpha \beta}^{\alpha^{\prime}} H_{\alpha^{\prime}}-P_{\alpha \beta} \\
Q_{\alpha \beta}= & -2 L_{\alpha \beta \alpha^{\prime}} u_{(4)}^{\alpha^{\prime}}+\frac{1}{4 k^{2}} R_{\alpha^{\prime} \alpha \beta \beta^{\prime}} H^{\alpha^{\prime}} H^{\beta^{\prime}}+\frac{1}{4 k^{2}} L_{\alpha \gamma}^{\alpha^{\prime}} L_{\beta}{ }_{\beta^{\prime}} H_{\alpha^{\prime}} H^{\beta^{\prime}} \\
& -\frac{1}{2 k} P_{\alpha \beta, \alpha^{\prime}} H^{\alpha^{\prime}}+\widetilde{B}_{\alpha \beta}-\frac{1}{k} P_{\alpha^{\prime}(\alpha} H_{, \beta)}^{\alpha^{\prime}}+\frac{1}{4 k^{2}} g_{\alpha^{\prime} \beta^{\prime}} H^{\alpha^{\prime}}{ }_{, \alpha} H^{\beta^{\prime}}{ }_{, \beta} \\
E= & \frac{1}{k^{2}}|H|^{2} \\
F= & -\frac{1}{k^{2}} P_{\alpha^{\prime} \beta^{\prime}} H^{\alpha^{\prime}} H^{\beta^{\prime}}+\frac{8}{k} H_{\alpha^{\prime}} u_{(4)}^{\alpha^{\prime}} .
\end{aligned}
$$

However, again by Lemma 5.2 and using (5.6) , at $p$ we have $H^{\alpha^{\prime}}{ }_{, \alpha}=\nabla_{\alpha} H^{\alpha^{\prime}}$ and $P_{\alpha \beta, \alpha^{\prime}}=$ ${ }^{M} \nabla_{\alpha^{\prime}} P_{\alpha \beta}-L_{\alpha \gamma \alpha^{\prime}} P_{\beta}^{\gamma}-L_{\beta \gamma \alpha^{\prime}} P^{\gamma}{ }_{\alpha}$. So $P_{\alpha \beta, \alpha^{\prime}} H^{\alpha^{\prime}}={ }^{M} \nabla_{\alpha^{\prime}} P_{\alpha \beta} H^{\alpha^{\prime}}-2 L_{\gamma(\alpha}^{\alpha^{\prime}} P_{\beta)}{ }^{\gamma} H_{\alpha^{\prime}}$. Thus the formula for $Q_{\alpha \beta}$ above becomes

$$
\begin{aligned}
Q_{\alpha \beta}= & -2 L_{\alpha \beta \alpha^{\prime}} u_{(4)}^{\alpha^{\prime}}+\frac{1}{4 k^{2}} R_{\alpha^{\prime} \alpha \beta \beta^{\prime}} H^{\alpha^{\prime}} H^{\beta^{\prime}}+\frac{1}{4 k^{2}} L_{\alpha \gamma}^{\alpha^{\prime}} L_{\beta}{ }_{\beta^{\prime}} H_{\alpha^{\prime}} H^{\beta^{\prime}}-\frac{1}{2 k}{ }^{M} \nabla_{\alpha^{\prime}} P_{\alpha \beta} H^{\alpha^{\prime}} \\
& +\frac{1}{k} L_{\gamma(\alpha}^{\alpha^{\prime}} P_{\beta)}{ }^{\gamma} H_{\alpha^{\prime}}+\widetilde{B}_{\alpha \beta}-\frac{1}{k} P_{\alpha^{\prime}(\alpha} \nabla_{\beta)} H^{\alpha^{\prime}}+\frac{1}{4 k^{2}} g_{\alpha^{\prime} \beta^{\prime}} \nabla_{\alpha} H^{\alpha^{\prime}} \nabla_{\beta} H^{\beta^{\prime}} .
\end{aligned}
$$

We need to calculate $\operatorname{det} \bar{h}_{\alpha \beta}$ to use in (5.11). Taylor expanding the determinant function shows that for $\bar{h}_{\alpha \beta}$ of the form (5.14), we have

$$
\operatorname{det} \bar{h}_{\alpha \beta}=\operatorname{det} g_{\alpha \beta}\left[1+D_{\alpha}{ }^{\alpha} r^{2}+\left(Q_{\alpha}{ }^{\alpha}-\frac{1}{2} D_{\alpha \beta} D^{\alpha \beta}+\frac{1}{2}\left(D_{\alpha}{ }^{\alpha}\right)^{2}\right) r^{4}+o\left(r^{4}\right)\right] .
$$

Multiplying by $\bar{h}_{00}$ and recalling (5.11), we get

$$
\begin{aligned}
\frac{\operatorname{det} \bar{h}}{\operatorname{det} g_{\alpha \beta}} & =\frac{\operatorname{det} \bar{h}_{\alpha \beta}}{\operatorname{det} g_{\alpha \beta}} \cdot \bar{h}_{00}+O\left(r^{6}\right) \\
& =1+\left(D_{\alpha}{ }^{\alpha}+E\right) r^{2}+\left(Q_{\alpha}{ }^{\alpha}-\frac{1}{2} D_{\alpha \beta} D^{\alpha \beta}+\frac{1}{2}\left(D_{\alpha}{ }^{\alpha}\right)^{2}+F+E D_{\alpha}{ }^{\alpha}\right) r^{4}+o\left(r^{4}\right) .
\end{aligned}
$$

Finally, using $\sqrt{1+x}=1+\frac{1}{2} x-\frac{1}{8} x^{2}+o\left(x^{2}\right)$ gives

$$
\begin{aligned}
\sqrt{\frac{\operatorname{det} \bar{h}}{\operatorname{det} g_{\alpha \beta}}=1} & +\frac{1}{2}\left(D_{\alpha}{ }^{\alpha}+E\right) r^{2} \\
& +\frac{1}{2}\left(Q_{\alpha}{ }^{\alpha}-\frac{1}{2} D_{\alpha \beta} D^{\alpha \beta}+\frac{1}{4}\left(D_{\alpha}{ }^{\alpha}\right)^{2}+F+\frac{1}{2} E D_{\alpha}{ }^{\alpha}-\frac{1}{4} E^{2}\right) r^{4}+o\left(r^{4}\right) .
\end{aligned}
$$

Recalling (4.3) and that $\bar{h}_{\alpha \beta}=g_{\alpha \beta}$ when $r=0$, we conclude

$$
\begin{aligned}
& a^{(2)}=\frac{1}{2}\left(D_{\alpha}{ }^{\alpha}+E\right) \\
& a^{(4)}=\frac{1}{2}\left(Q_{\alpha}{ }^{\alpha}-\frac{1}{2} D_{\alpha \beta} D^{\alpha \beta}+\frac{1}{4}\left(D_{\alpha}{ }^{\alpha}\right)^{2}+F+\frac{1}{2} E D_{\alpha}{ }^{\alpha}-\frac{1}{4} E^{2}\right) .
\end{aligned}
$$

The formula for $a^{(2)}$ in Proposition 5.1 follows upon substituting (5.15) for $D_{\alpha \beta}$ and $E$. To obtain the formula for $a^{(4)}$, one substitutes (5.15) for $D_{\alpha \beta}, E$, and $F$, (5.16) for $Q_{\alpha \beta}$, (5.9) for $\widetilde{B}_{\alpha \beta}$ in (5.16), writes $R_{\alpha^{\prime} \alpha \beta \beta^{\prime}}$ in (5.16) in terms of the Weyl and Schouten tensors via (2.1), and collects terms. 
Corollary 5.3. If $k=2$, then

$$
\mathcal{E}=-\frac{1}{8} \int_{\Sigma}\left(|H|^{2}+4 P^{\alpha}{ }_{\alpha}\right) d a_{\Sigma}
$$

If $k=4$, then

$$
\begin{aligned}
\mathcal{E}=\frac{1}{128} \int_{\Sigma}( & |\nabla H|^{2}-L_{\alpha \beta}^{\alpha^{\prime}} L_{\beta^{\prime}}^{\alpha \beta} H_{\alpha^{\prime}} H^{\beta^{\prime}}+\frac{7}{16}|H|^{4} \\
& -W^{\alpha}{ }_{\alpha^{\prime} \alpha \beta^{\prime}} H^{\alpha^{\prime}} H^{\beta^{\prime}}-8 P^{\alpha}{ }_{\alpha^{\prime}} \nabla_{\alpha} H^{\alpha^{\prime}}-8 C^{\alpha}{ }_{\alpha \alpha^{\prime}} H^{\alpha^{\prime}}-8 P^{\alpha \beta} L_{\alpha \beta}^{\alpha^{\prime}} H_{\alpha^{\prime}}+5 P^{\alpha}{ }_{\alpha}|H|^{2} \\
& \left.-16 P^{\alpha \beta} P_{\alpha \beta}+16 P^{\alpha \alpha^{\prime}} P_{\alpha \alpha^{\prime}}+16\left(P^{\alpha}{ }_{\alpha}\right)^{2}-\frac{16}{n-4} B^{\alpha}{ }_{\alpha}\right) d a_{\Sigma}
\end{aligned}
$$

Proof. Recall that $\mathcal{E}=\int_{\Sigma} a^{(k)} d a_{\Sigma}$ for $\Sigma$ of dimension $k$. The result for $k=2$ follows immediately upon setting $k=2$ in Proposition 5.1 and integrating. For $k=4$, integrating the formula in Proposition 5.1 and comparing with that in Corollary 5.3 shows that the result reduces to the following identity:

$$
\begin{aligned}
\int_{\Sigma}\left(g^{\alpha \beta M} \nabla_{\alpha^{\prime}} P_{\alpha \beta} H^{\alpha^{\prime}}+P^{\alpha}{ }_{\alpha^{\prime}} \nabla_{\alpha} H^{\alpha^{\prime}}\right. & \left.+P_{\alpha^{\prime} \beta^{\prime}} H^{\alpha^{\prime}} H^{\beta^{\prime}}\right) d a_{\Sigma} \\
& =\int_{\Sigma}\left(C^{\alpha}{ }_{\alpha \alpha^{\prime}} H^{\alpha^{\prime}}+P^{\alpha \beta} L_{\alpha \beta}^{\alpha^{\prime}} H_{\alpha^{\prime}}\right) d a_{\Sigma} .
\end{aligned}
$$

We intend to integrate the $\nabla_{\alpha}$ by parts in the second term on the left-hand side to obtain $-\nabla_{\alpha} P^{\alpha}{ }_{\alpha^{\prime}} H^{\alpha^{\prime}}$. This $\nabla_{\alpha}$ denotes the normal bundle connection, so we are obliged here to interpret $P^{\alpha} \alpha_{\alpha^{\prime}}$ as a section of $T \Sigma \otimes N^{*} \Sigma$ on $\Sigma$ and $\nabla_{\alpha}$ as the induced connection on this bundle. Recalling (5.6), we have

$$
\nabla_{\alpha} P_{\beta \alpha^{\prime}}={ }^{M} \nabla_{\alpha} P_{\beta \alpha^{\prime}}+\Gamma_{\alpha \beta}^{\beta^{\prime}} P_{\beta^{\prime} \alpha^{\prime}}+\Gamma_{\alpha \alpha^{\prime}}^{\gamma} P_{\beta \gamma}={ }^{M} \nabla_{\alpha} P_{\beta \alpha^{\prime}}+L_{\alpha \beta}^{\beta^{\prime}} P_{\beta^{\prime} \alpha^{\prime}}-L_{\alpha \alpha^{\prime}}^{\gamma} P_{\beta \gamma} .
$$

Thus

$$
-\nabla_{\alpha} P_{\alpha^{\prime}}^{\alpha} H^{\alpha^{\prime}}=-g^{\alpha \beta M} \nabla_{\alpha} P_{\beta \alpha^{\prime}} H^{\alpha^{\prime}}-P_{\alpha^{\prime} \beta^{\prime}} H^{\alpha^{\prime}} H^{\beta^{\prime}}+L_{\alpha \beta}^{\alpha^{\prime}} P^{\alpha \beta} H_{\alpha^{\prime}} .
$$

So integrating by parts as described and substituting for $-\nabla_{\alpha} P^{\alpha}{ }_{\alpha^{\prime}} H^{\alpha^{\prime}}$, one concludes that the left-hand side of (5.18) equals

$$
\begin{aligned}
\int_{\Sigma}\left(g^{\alpha \beta M} \nabla_{\alpha^{\prime}} P_{\alpha \beta} H^{\alpha^{\prime}}-g^{\alpha \beta M} \nabla_{\alpha} P_{\beta \alpha^{\prime}} H^{\alpha^{\prime}}\right. & \left.+L_{\alpha \beta}^{\alpha^{\prime}} P^{\alpha \beta} H_{\alpha^{\prime}}\right) d a_{\Sigma} \\
& =\int_{\Sigma}\left(C^{\alpha}{ }_{\alpha \alpha^{\prime}} H^{\alpha^{\prime}}+P^{\alpha \beta} L_{\alpha \beta}^{\alpha^{\prime}} H_{\alpha^{\prime}}\right) d a_{\Sigma}
\end{aligned}
$$

as desired.

Remark 5.4. The coefficients in Proposition 5.1 and Corollary 5.3 become simpler when written in terms of the alternate convention for the mean curvature: $\bar{H}=\frac{1}{k} H$. In particular, 
one has

$$
\begin{aligned}
a^{(4)}=\frac{1}{8} & \left(|\nabla \bar{H}|^{2}-L_{\alpha \beta}^{\alpha^{\prime}} L_{\beta^{\prime}}^{\alpha \beta} \bar{H}_{\alpha^{\prime}} \bar{H}^{\beta^{\prime}}+\left(k^{2}-2 k-1\right)|\bar{H}|^{4}\right. \\
& -W^{\alpha}{ }_{\alpha^{\prime} \alpha \beta^{\prime}} \bar{H}^{\alpha^{\prime}} \bar{H}^{\beta^{\prime}}-2 g^{\alpha \beta M} \nabla_{\alpha^{\prime}} P_{\alpha \beta} \bar{H}^{\alpha^{\prime}}-4 P^{\alpha}{ }_{\alpha^{\prime}} \nabla_{\alpha} \bar{H}^{\alpha^{\prime}} \\
& +(2 k-3) P^{\alpha}{ }_{\alpha}|\bar{H}|^{2}-(k+4) P_{\alpha^{\prime} \beta^{\prime}} \bar{H}^{\alpha^{\prime}} \bar{H}^{\beta^{\prime}} \\
& \left.-P^{\alpha \beta} P_{\alpha \beta}+P^{\alpha \alpha^{\prime}} P_{\alpha \alpha^{\prime}}+\left(P^{\alpha}{ }_{\alpha}\right)^{2}-\frac{1}{n-4} B^{\alpha}{ }_{\alpha}\right) \\
& +(4-k) \bar{H}_{\alpha^{\prime}} U_{(4)}^{\alpha^{\prime}}
\end{aligned}
$$

and for $k=4$

$$
\begin{aligned}
\mathcal{E}=\frac{1}{8} \int_{\Sigma}( & |\nabla \bar{H}|^{2}-L_{\alpha \beta}^{\alpha^{\prime}} L_{\beta^{\prime}}^{\alpha \beta} \bar{H}_{\alpha^{\prime}} \bar{H}^{\beta^{\prime}}+7|\bar{H}|^{4} \\
& \quad-W^{\alpha}{ }_{\alpha^{\prime} \alpha \beta^{\prime}} \bar{H}^{\alpha^{\prime}} \bar{H}^{\beta^{\prime}}-2 P^{\alpha}{ }_{\alpha^{\prime}} \nabla_{\alpha} \bar{H}^{\alpha^{\prime}}-2 C^{\alpha}{ }_{\alpha \alpha^{\prime}} \bar{H}^{\alpha^{\prime}}-2 P^{\alpha \beta} L_{\alpha \beta}^{\alpha^{\prime}} \bar{H}_{\alpha^{\prime}}+5 P^{\alpha}{ }_{\alpha}|\bar{H}|^{2} \\
& \left.\quad-P^{\alpha \beta} P_{\alpha \beta}+P^{\alpha \alpha^{\prime}} P_{\alpha \alpha^{\prime}}+\left(P^{\alpha}{ }_{\alpha}\right)^{2}-\frac{1}{n-4} B^{\alpha}{ }_{\alpha}\right) d a_{\Sigma} .
\end{aligned}
$$

Recall that equation (5.1) identifies $U_{(2)}$. The next proposition identifies $U_{(4)}$.

Proposition 5.5. If $k>2$, then

$$
\begin{aligned}
U_{(4)}^{\alpha^{\prime}}=\frac{1}{8 k(k-2)} & \left((\Delta H)^{\alpha^{\prime}}+L_{\alpha \beta}^{\alpha^{\prime}} L_{\beta^{\prime}}^{\alpha \beta} H^{\beta^{\prime}}-\frac{2}{k^{2}}|H|^{2} H^{\alpha^{\prime}}\right. \\
& +W^{\alpha \alpha^{\prime}}{ }_{\alpha \beta^{\prime}} H^{\beta^{\prime}}-P^{\alpha}{ }_{\alpha} H^{\alpha^{\prime}}+(k-4) P^{\alpha^{\prime}{ }_{\beta^{\prime}}} H^{\beta^{\prime}}+2 k P^{\alpha \beta} L_{\alpha \beta}^{\alpha^{\prime}} \\
& \left.+k g^{\alpha^{\prime} \beta^{\prime}} g^{\alpha \beta}\left({ }^{M} \nabla_{\beta^{\prime}} P_{\alpha \beta}-2^{M} \nabla_{\alpha} P_{\beta \beta^{\prime}}\right)\right)
\end{aligned}
$$

If $k=2$, then

$$
\begin{aligned}
\mathcal{H}^{\alpha^{\prime}}=\frac{1}{4} & \left((\Delta H)^{\alpha^{\prime}}+L_{\alpha \beta}^{\alpha^{\prime}} L_{\beta^{\prime}}^{\alpha \beta} H^{\beta^{\prime}}-\frac{1}{2}|H|^{2} H^{\alpha^{\prime}}\right. \\
& +W^{\alpha \alpha^{\prime}}{ }_{\alpha \beta^{\prime}} H^{\beta^{\prime}}-P^{\alpha}{ }_{\alpha} H^{\alpha^{\prime}}-2 P^{\alpha^{\prime}}{ }_{\beta^{\prime}} H^{\beta^{\prime}}+4 P^{\alpha \beta} L_{\alpha \beta}^{\alpha^{\prime}} \\
& \left.+2 g^{\alpha^{\prime} \beta^{\prime}} g^{\alpha \beta}\left({ }^{M} \nabla_{\beta^{\prime}} P_{\alpha \beta}-2^{M} \nabla_{\alpha} P_{\beta \beta^{\prime}}\right)\right)
\end{aligned}
$$

Proof. Just as in the proof of Proposition 5.1, since these formulas are coordinate-invariant, it suffices to prove them at $p$ in our special adapted coordinates $(x, u)$. Now $U_{(4)}$ is determined by applying $\left.\partial_{r}^{3}\right|_{r=0}$ to the equation $\mathcal{M}(u)=0$, with $\mathcal{M}(u)$ given by (3.5). Recall that in 
(3.5), we have $\bar{h}^{0 \alpha}=O\left(r^{3}\right), g_{\alpha \gamma^{\prime}}=O\left(r^{2}\right)$ and $u^{\alpha^{\prime}}=O\left(r^{2}\right)$. Therefore

$$
\begin{aligned}
\mathcal{M}(u)_{\gamma^{\prime}}= & \left(r \partial_{r}-(k+1)+\frac{1}{2} r \mathcal{L}_{, r}\right)\left(\bar{h}^{00} g_{\alpha^{\prime} \gamma^{\prime}} u^{\alpha^{\prime}, r}\right) \\
& +r\left(\partial_{\beta}+\frac{1}{2} \mathcal{L}_{, \beta}\right)\left[\bar{h}^{\alpha \beta}\left(g_{\alpha \gamma^{\prime}}+g_{\alpha^{\prime} \gamma^{\prime}} u^{\alpha^{\prime}, \alpha}\right)\right] \\
& -\frac{1}{2} r \bar{h}^{\alpha \beta}\left(g_{\alpha \beta, \gamma^{\prime}}+2 g_{\alpha \alpha^{\prime}, \gamma^{\prime}} u^{\alpha^{\prime}, \beta}\right)-\frac{1}{2} r \bar{h}^{00} g_{\alpha^{\prime} \beta^{\prime}, \gamma^{\prime}} u^{\alpha^{\prime}{ }_{, r}} u^{\beta^{\prime}}{ }_{, r}+O\left(r^{5}\right) .
\end{aligned}
$$

Apply $\left.\partial_{r}^{3}\right|_{r=0}$ to the equation $\mathcal{M}(u)=0$. Keeping in mind the orders of vanishing and the parity of the various terms and the fact that $\bar{h}^{00}=1$ on $\Sigma$, one obtains at $r=0$ :

$$
\begin{aligned}
0= & (2-k) \partial_{r}^{3}\left(\bar{h}^{00} g_{\alpha^{\prime} \gamma^{\prime}} u^{\alpha^{\prime}}{ }_{, r}\right)+3 \mathcal{L}_{, r r} g_{\alpha^{\prime} \gamma^{\prime}} u^{\alpha^{\prime}}{ }_{, r r} \\
& +3\left(\partial_{\beta}+\frac{1}{2} \mathcal{L}_{, \beta}\right)\left[\bar{h}^{\alpha \beta}\left(\partial_{r}^{2} g_{\alpha \gamma^{\prime}}+g_{\alpha^{\prime} \gamma^{\prime}} u^{\alpha^{\prime}}{ }_{, \alpha r r}\right)\right] \\
& -\frac{3}{2} \bar{h}^{\alpha \beta}\left(\partial_{r}^{2} g_{\alpha \beta, \gamma^{\prime}}+2 g_{\alpha \alpha^{\prime}, \gamma^{\prime}} u_{, \beta r r}^{\alpha^{\prime}}\right)-\frac{3}{2}\left(\partial_{r}^{2} \bar{h}^{\alpha \beta}\right) g_{\alpha \beta, \gamma^{\prime}}-3 g_{\alpha^{\prime} \beta^{\prime}, \gamma^{\prime}} u^{\alpha^{\prime}{ }_{, r r} u^{\beta^{\prime}{ }_{, r r} .}}
\end{aligned}
$$

Expanding the derivatives gives

$$
\partial_{r}^{3}\left(\bar{h}^{00} g_{\alpha^{\prime} \gamma^{\prime}} u^{\alpha^{\prime}{ }_{, r}}\right)=g_{\alpha^{\prime} \gamma^{\prime}} \partial_{r}^{4} u^{\alpha^{\prime}}+3\left(g_{\alpha^{\prime} \gamma^{\prime}} \partial_{r}^{2} \bar{h}^{00}+\partial_{r}^{2} g_{\alpha^{\prime} \gamma^{\prime}}\right) u_{, r r}^{\alpha^{\prime}}
$$

at $r=0$. Since $\bar{h}_{\alpha \beta}=g_{\alpha \beta}$ on $\Sigma$, it follows from Lemma 5.2 that $\mathcal{L}_{, \beta}=g^{\alpha \gamma} g_{\alpha \gamma, \beta}=0$ at $p$. Lemma 5.2 also implies that $\partial_{\beta} \bar{h}^{\alpha \beta}, g_{\alpha \alpha^{\prime}, \gamma^{\prime}}$ and $g_{\alpha^{\prime} \beta^{\prime}, \gamma^{\prime}}$ vanish at $p$. So evaluating (5.19) at $p$ and solving for $\partial_{r}^{4} u^{\alpha^{\prime}}$ yield

$$
\begin{aligned}
(k-2) g_{\alpha^{\prime} \gamma^{\prime}} \partial_{r}^{4} u^{\alpha^{\prime}}= & 3(2-k)\left(g_{\alpha^{\prime} \gamma^{\prime}} \partial_{r}^{2} \bar{h}^{00}+\partial_{r}^{2} g_{\alpha^{\prime} \gamma^{\prime}}\right) u^{\alpha^{\prime}{ }_{, r r}}+3 \mathcal{L}_{, r r} g_{\alpha^{\prime} \gamma^{\prime}} u^{\alpha^{\prime}{ }_{, r r}} \\
& +3 g^{\alpha \beta} \partial_{\beta}\left(\partial_{r}^{2} g_{\alpha \gamma^{\prime}}+g_{\alpha^{\prime} \gamma^{\prime}} u^{\alpha^{\prime}}{ }_{, \alpha r r}\right) \\
& -\frac{3}{2} g^{\alpha \beta} \partial_{r}^{2} g_{\alpha \beta, \gamma^{\prime}}-\frac{3}{2}\left(\partial_{r}^{2} \bar{h}^{\alpha \beta}\right) g_{\alpha \beta, \gamma^{\prime}} .
\end{aligned}
$$

Expanding the derivative on the second line and reordering terms gives

$$
\begin{aligned}
(k-2) g_{\alpha^{\prime} \gamma^{\prime}} \partial_{r}^{4} u^{\alpha^{\prime}=}= & 3 g_{\alpha^{\prime} \gamma^{\prime}} g^{\alpha \beta} u^{\alpha^{\prime}}{ }_{, r r \alpha \beta}+3 g^{\alpha \beta} \partial_{\beta} \partial_{r}^{2} g_{\alpha \gamma^{\prime}} \\
& +3(2-k) g_{\alpha^{\prime} \gamma^{\prime}}\left(\partial_{r}^{2} \bar{h}^{00}\right) u^{\alpha^{\prime}{ }_{, r r}}+3(2-k)\left(\partial_{r}^{2} g_{\alpha^{\prime} \gamma^{\prime}}\right) u^{\alpha^{\prime}{ }_{, r r}} \\
& +3 \mathcal{L}{ }_{, r r} g_{\alpha^{\prime} \gamma^{\prime}} u^{\alpha^{\prime}{ }_{, r r}}-\frac{3}{2} g^{\alpha \beta} \partial_{r}^{2} g_{\alpha \beta, \gamma^{\prime}}-\frac{3}{2}\left(\partial_{r}^{2} \bar{h}^{\alpha \beta}\right) g_{\alpha \beta, \gamma^{\prime}} .
\end{aligned}
$$

We have already evaluated many of the ingredients on the right-hand side. For instance, $g_{\alpha \beta, \gamma^{\prime}}=-2 L_{\alpha \beta \gamma^{\prime}}$ by (5.2) and $\partial_{r}^{2} u^{\alpha^{\prime}}=\frac{1}{k} H^{\alpha^{\prime}}$ by (5.1). Also $\partial_{r}^{2} \bar{h}^{00}=-2 E$ and $\partial_{r}^{2} \bar{h}^{\alpha \beta}=$ $-2 D^{\alpha \beta}$ by (5.14). Similarly, taking the log and differentiating in (5.17) gives $\mathcal{L}_{, r r}=2\left(D_{\alpha}{ }^{\alpha}+\right.$ $E)$. We have $\partial_{r}^{2} g_{\alpha^{\prime} \gamma^{\prime}}=-2 P_{\alpha^{\prime} \gamma^{\prime}}$ by (5.12). The terms that still need to be evaluated are $g^{\alpha \beta} u^{\alpha^{\prime}}{ }_{, r r \alpha \beta}, \partial_{\beta} \partial_{r}^{2} g_{\alpha \gamma^{\prime}}$ and $\partial_{r}^{2} g_{\alpha \beta, \gamma^{\prime}}$. Using (5.4), we have at $p$ :

$$
g^{\alpha \beta} u_{, r r \alpha \beta}^{\alpha^{\prime}}=\frac{1}{k} g^{\alpha \beta} H_{, \alpha \beta}^{\alpha^{\prime}}=\frac{1}{k} \Delta H^{\alpha^{\prime}}-\frac{1}{k} g^{\alpha^{\prime} \gamma^{\prime}} g^{\alpha \beta} g_{\alpha \gamma^{\prime}, \beta \beta^{\prime}} H^{\beta^{\prime}} .
$$


For $\partial_{\beta} \partial_{r}^{2} g_{\alpha \gamma^{\prime}}$ and $\partial_{r}^{2} g_{\alpha \beta, \gamma^{\prime}}$, recall that in (3.5), all components of $g$ and its derivatives which appear are evaluated at $\left(x, u(x, r, r)\right.$. We can evaluate $\partial_{r}^{2} g_{\alpha \beta, \gamma^{\prime}}$ by the same procedure we used in the proof of Proposition 5.1. First differentiate (5.8) to obtain

$$
g_{\alpha \beta, \gamma^{\prime}}(x, u, r)=g_{\alpha \beta, \gamma^{\prime}}(x, u, 0)-P_{\alpha \beta, \gamma^{\prime}} r^{2}+o\left(r^{2}\right) .
$$

Evaluate at $u=u(x, r)$ :

$$
\begin{aligned}
g_{\alpha \beta, \gamma^{\prime}}(x, u(x, r), r) & =g_{\alpha \beta, \gamma^{\prime}}(x, u(x, r), 0)-P_{\alpha \beta, \gamma^{\prime}} r^{2}+o\left(r^{2}\right) \\
& =g_{\alpha \beta, \gamma^{\prime}}(x, 0,0)+g_{\alpha \beta, \gamma^{\prime} \beta^{\prime}}(x, 0,0) u^{\beta^{\prime}}(x, r)-P_{\alpha \beta, \gamma^{\prime}} r^{2}+o\left(r^{2}\right) \\
& =g_{\alpha \beta, \gamma^{\prime}}(x, 0,0)+\frac{1}{2 k} g_{\alpha \beta, \gamma^{\prime} \beta^{\prime}} H^{\beta^{\prime}} r^{2}-P_{\alpha \beta, \gamma^{\prime}} r^{2}+o\left(r^{2}\right) .
\end{aligned}
$$

Thus

$$
\partial_{r}^{2} g_{\alpha \beta, \gamma^{\prime}}=\frac{1}{k} g_{\alpha \beta, \gamma^{\prime} \beta^{\prime}} H^{\beta^{\prime}}-2 P_{\alpha \beta, \gamma^{\prime}}
$$

For $\partial_{\beta} \partial_{r}^{2} g_{\alpha \gamma^{\prime}}$, begin as above by differentiating (5.8):

$$
g_{\alpha \gamma^{\prime}, \beta}(x, u, r)=g_{\alpha \gamma^{\prime}, \beta}(x, u, 0)-P_{\alpha \gamma^{\prime}, \beta} r^{2}+o\left(r^{2}\right) .
$$

So the chain rule gives

$$
\begin{aligned}
\partial_{\beta}\left(g_{\alpha \gamma^{\prime}}(x, u(x, r), r)\right)=g_{\alpha \gamma^{\prime}, \beta}(x, u(x, r), r)+g_{\alpha \gamma^{\prime}, \beta^{\prime}}(x, u(x, r), r) u_{, \beta}^{\beta^{\prime}}(x, r) \\
\quad=g_{\alpha \gamma^{\prime}, \beta}(x, u(x, r), 0)-P_{\alpha \gamma^{\prime}, \beta} r^{2}+\frac{1}{2 k} g_{\alpha \gamma^{\prime}, \beta^{\prime}}(x, 0,0) H_{, \beta}^{\beta^{\prime}} r^{2}+o\left(r^{2}\right) \\
\quad=g_{\alpha \gamma^{\prime}, \beta}(x, 0,0)+g_{\alpha \gamma^{\prime}, \beta \beta^{\prime}} u^{\beta^{\prime}}(x, r)-P_{\alpha \gamma^{\prime}, \beta} r^{2}+\frac{1}{2 k} g_{\alpha \gamma^{\prime}, \beta^{\prime}}(x, 0,0) H_{, \beta}^{\beta^{\prime}} r^{2}+o\left(r^{2}\right) \\
\quad=g_{\alpha \gamma^{\prime}, \beta}(x, 0,0)+\frac{1}{2 k} g_{\alpha \gamma^{\prime}, \beta \beta^{\prime}} H^{\beta^{\prime}} r^{2}-P_{\alpha \gamma^{\prime}, \beta} r^{2}+\frac{1}{2 k} g_{\alpha \gamma^{\prime}, \beta^{\prime}}(x, 0,0) H_{, \beta}^{\beta^{\prime}} r^{2}+o\left(r^{2}\right) .
\end{aligned}
$$

At $x=0$, corresponding to the point $p$, we have $g_{\alpha \gamma^{\prime}, \beta^{\prime}}=0$. Thus at $p$ we obtain

$$
\partial_{\beta} \partial_{r}^{2} g_{\alpha \gamma^{\prime}}=\frac{1}{k} g_{\alpha \gamma^{\prime}, \beta \beta^{\prime}} H^{\beta^{\prime}}-2 P_{\alpha \gamma^{\prime}, \beta} \text {. }
$$

Now substitute all of these into (5.20) and raise the free index. The two terms involving $g_{\alpha \gamma^{\prime}, \beta \beta^{\prime}}$ cancel, and one obtains

$$
\begin{aligned}
(k-2) \partial_{r}^{4} u^{\alpha^{\prime}}= & \frac{3}{k} \Delta H^{\alpha^{\prime}}+3 g^{\alpha^{\prime} \gamma^{\prime}} g^{\alpha \beta}\left(P_{\alpha \beta, \gamma^{\prime}}-2 P_{\alpha \gamma^{\prime}, \beta}\right)-\frac{6(2-k)}{k} E H^{\alpha^{\prime}}-\frac{6(2-k)}{k} P_{\beta^{\prime}}^{\alpha^{\prime}} H^{\beta^{\prime}} \\
& +\frac{6}{k}\left(D^{\alpha}{ }_{\alpha}+E\right) H^{\alpha^{\prime}}-\frac{3}{2 k} g^{\alpha^{\prime} \gamma^{\prime}} g^{\alpha \beta} g_{\alpha \beta, \gamma^{\prime} \beta^{\prime}} H^{\beta^{\prime}}-6 D^{\alpha \beta} L_{\alpha \beta}^{\alpha^{\prime}} .
\end{aligned}
$$

Expanding the covariant derivatives in terms of partial derivatives and Christoffel symbols shows that at $p$ :

$$
g^{\alpha \beta}\left(P_{\alpha \beta, \gamma^{\prime}}-2 P_{\alpha \gamma^{\prime}, \beta}\right)=g^{\alpha \beta}\left({ }^{M} \nabla_{\gamma^{\prime}} P_{\alpha \beta}-2^{M} \nabla_{\beta} P_{\alpha \gamma^{\prime}}\right)-2 P_{\gamma^{\prime} \beta^{\prime}} H^{\beta^{\prime}} .
$$

The formula for $U_{(4)}$ in Proposition 5.5 is obtained as follows. In (5.21), substitute (5.22) for $g^{\alpha \beta}\left(P_{\alpha \beta, \gamma^{\prime}}-2 P_{\alpha \gamma^{\prime}, \beta}\right)$, substitute (5.15) for $D_{\alpha \beta}$ and $E$, substitute (5.3) for $g_{\alpha \beta, \gamma^{\prime} \beta^{\prime}}$, write the resulting $R_{\gamma^{\prime} \alpha \beta \beta^{\prime}}$ in terms of the Weyl and Schouten tensors via (2.1), collect terms, and finally note that $U_{(4)}=\left.\frac{1}{24} \partial_{r}^{4} U\right|_{r=0}$. 
Equation (5.21) confirms that $\left.\partial_{r}^{3} \mathcal{M}(u)\right|_{r=0}$ is independent of $\left.\partial_{r}^{4} u^{\alpha^{\prime}}\right|_{r=0}$ when $k=2$. In this case, the above calculation shows that $\left.g^{\alpha^{\prime} \gamma^{\prime}} \partial_{r}^{3} \mathcal{M}(u)_{\gamma^{\prime}}\right|_{r=0}$ equals the right-hand side of (5.21). However, by (3.8) we have

$$
\left.g^{\alpha^{\prime} \gamma^{\prime}} \partial_{r}^{3} \mathcal{M}(u)_{\gamma^{\prime}}\right|_{r=0}=\left.\partial_{r}^{3}\left(r^{3} \mathcal{H}^{\alpha^{\prime}}\right)\right|_{r=0}=6 \mathcal{H}^{\alpha^{\prime}} .
$$

This gives the formula for $\mathcal{H}$ in Proposition 5.5.

We have also calculated the variation for $k=4$ when the background $M$ is $\mathbb{R}^{n}$ with the Euclidean metric. In [Gu1, Gu2], Guven developed a very nice formalism for identifying the variation of functionals of hypersurfaces in Euclidean space, using Lagrange multipliers to encode the data of the embedding. As he suggested, it is straightforward to extend his formalism to submanifolds of higher codimension. We refer to his papers for the details of the method and formulate the following consequences.

Let $S\left(g_{\alpha \beta}, g_{\alpha^{\prime} \beta^{\prime}}, L_{\alpha \beta \alpha^{\prime}}\right)$ be a smooth function of $g_{\alpha \beta} \in S_{+}^{2} \mathbb{R}^{k *}, g_{\alpha^{\prime} \beta^{\prime}} \in S_{+}^{2} \mathbb{R}^{n-k *}$, and $L_{\alpha \beta \alpha^{\prime}} \in S^{2} \mathbb{R}^{k *} \otimes \mathbb{R}^{n-k *}$. Here $S_{+}^{2} \mathbb{R}^{l *}$ denotes the cone of positive definite symmetric bilinear forms on $\mathbb{R}^{l}$. It is assumed that $S$ is invariant under the natural action of $G L(k, \mathbb{R}) \times G L(n-$ $k, \mathbb{R})$ on $S_{+}^{2} \mathbb{R}^{k *} \oplus S_{+}^{2} \mathbb{R}^{n-k *} \oplus\left(S^{2} \mathbb{R}^{k *} \otimes \mathbb{R}^{n-k *}\right)$. Let $\Sigma$ be a compact $k$-manifold and let

$$
\mathcal{F}=\int_{\Sigma} S\left(g_{\alpha \beta}, g_{\alpha^{\prime} \beta^{\prime}}, L_{\alpha \beta \alpha^{\prime}}\right) d a_{\Sigma}
$$

be a functional on the space of immersions of $\Sigma$ into $\mathbb{R}^{n}$, where at each point $g_{\alpha \beta}$ is taken to be the induced metric, $g_{\alpha^{\prime} \beta^{\prime}}$ the metric on the normal space, and $L_{\alpha \beta \alpha^{\prime}}$ the second fundamental form with all indices lowered, all written in some choice of local frames for $T \Sigma$ and for $N \Sigma$. By the assumed invariance of $S$, the integrand is independent of the choice of frames and is a globally defined density on $\Sigma$ depending on the immersion $f$.

As in Theorem 4.3, let $F: \Sigma \times[0, \delta) \rightarrow \mathbb{R}^{n}$ be a variation of $\Sigma$ with infinitesimal variation $\dot{F} \in \Gamma\left(f^{*} T M\right)$. Guven's method shows that

$$
\dot{\mathcal{F}}=\int_{\Sigma}\langle\dot{F}, \mathcal{K}\rangle_{g} d a_{\Sigma}
$$

where $\mathcal{K}$ is the section of $N \Sigma$ given by

$$
\mathcal{K}^{\alpha^{\prime}}=\nabla_{\alpha} \nabla_{\beta}\left(\frac{\partial S}{\partial L_{\alpha \beta \alpha^{\prime}}}\right)+L_{\alpha \gamma}^{\alpha^{\prime}} L_{\beta \beta^{\prime}}^{\gamma} \frac{\partial S}{\partial L_{\alpha \beta \beta^{\prime}}}-S H^{\alpha^{\prime}} .
$$

For instance, for $S=1, \mathcal{F}$ is the area functional and $\mathcal{K}=-H$ is the negative of the mean curvature. For another example, the integrand for the Willmore energy is

$$
S=|H|^{2}=g^{\alpha^{\prime} \beta^{\prime}} g^{\alpha \beta} g^{\gamma \delta} L_{\alpha \beta \alpha^{\prime}} L_{\gamma \delta \beta^{\prime}} .
$$

So $\frac{\partial S}{\partial L_{\alpha \beta \alpha^{\prime}}}=2 g^{\alpha \beta} H^{\alpha^{\prime}}$, and (5.24) gives the usual formula for its variation:

$$
\mathcal{K}^{\alpha^{\prime}}=2 \Delta H^{\alpha^{\prime}}+2 L_{\alpha \beta}^{\alpha^{\prime}} L_{\beta^{\prime}}^{\alpha \beta} H^{\beta^{\prime}}-|H|^{2} H^{\alpha^{\prime}}
$$

We apply Guven's formalism to the energy functional $\mathcal{E}$ for $k=4$. If $M=\mathbb{R}^{n}$, then all background curvature vanishes, so the formula in Corollary 5.3 for the $k=4$ energy becomes 
(1.1). Consider separately the variation of each of the three terms in the integrand. For $S=|H|^{4}$, we have $\frac{\partial S}{\partial L_{\alpha \beta \alpha^{\prime}}}=4 g^{\alpha \beta}|H|^{2} H^{\alpha^{\prime}}$, so

$$
\mathcal{K}^{\alpha^{\prime}}=4 \Delta\left(|H|^{2} H^{\alpha^{\prime}}\right)+4 L_{\alpha \beta}^{\alpha^{\prime}} L_{\beta^{\prime}}^{\alpha \beta}|H|^{2} H^{\beta^{\prime}}-|H|^{4} H^{\alpha^{\prime}} .
$$

For $S=L_{\alpha \beta}^{\alpha^{\prime}} L_{\beta^{\prime}}^{\alpha \beta} H_{\alpha^{\prime}} H^{\beta^{\prime}}$, we have $\frac{\partial S}{\partial L_{\alpha \beta \alpha^{\prime}}}=2 g^{\alpha \beta} L_{\gamma \delta}^{\alpha^{\prime}} L_{\beta^{\prime}}^{\gamma \delta} H^{\beta^{\prime}}+2 L^{\alpha \beta \beta^{\prime}} H_{\beta^{\prime}} H^{\alpha^{\prime}}$, so

$$
\begin{aligned}
\mathcal{K}^{\alpha^{\prime}} & =2 \Delta\left(L_{\alpha \beta}^{\alpha^{\prime}} L_{\beta^{\prime}}^{\alpha \beta} H^{\beta^{\prime}}\right)+2 \nabla_{\alpha} \nabla_{\beta}\left(L^{\alpha \beta \beta^{\prime}} H_{\beta^{\prime}} H^{\alpha^{\prime}}\right) \\
& +2 L_{\gamma \delta}^{\alpha^{\prime}} L_{\beta^{\prime}}^{\gamma \delta} L_{\alpha \beta \gamma^{\prime}} L^{\alpha \beta \beta^{\prime}} H^{\gamma^{\prime}}+2 L_{\alpha \gamma}^{\alpha^{\prime}} L_{\beta \beta^{\prime}}^{\gamma} L^{\alpha \beta \gamma^{\prime}} H^{\beta^{\prime}} H_{\gamma^{\prime}}-L_{\alpha \beta}^{\gamma^{\prime}} L_{\beta^{\prime}}^{\alpha \beta} H_{\gamma^{\prime}} H^{\beta^{\prime}} H^{\alpha^{\prime}} .
\end{aligned}
$$

In addition to functionals of the form (5.23), Guven's formalism applies to functionals involving covariant derivatives of $L_{\alpha \beta}^{\alpha^{\prime}}$. For $S=|\nabla H|^{2}$, it gives (see [Gu2]):

$$
\mathcal{K}^{\alpha^{\prime}}=-2 \Delta^{2} H^{\alpha^{\prime}}+2 L^{\alpha \beta \alpha^{\prime}} \nabla_{\alpha} H^{\beta^{\prime}} \nabla_{\beta} H_{\beta^{\prime}}-2 L^{\alpha \beta \alpha^{\prime}} L_{\alpha \beta \beta^{\prime}} \Delta H^{\beta^{\prime}}-|\nabla H|^{2} H^{\alpha^{\prime}} .
$$

Recalling that Theorem 4.3 identifies $\mathcal{H}$ as the negative of the variation of $\mathcal{E}$ and combining the above ingredients yields the following.

Proposition 5.6. When $k=4$ and $M=\mathbb{R}^{n}$ with the Euclidean metric, we have

$$
\begin{aligned}
128 \mathcal{H}^{\alpha^{\prime}} & =2 \Delta^{2} H^{\alpha^{\prime}}-2 L^{\alpha \beta \alpha^{\prime}} \nabla_{\alpha} H^{\beta^{\prime}} \nabla_{\beta} H_{\beta^{\prime}}+2 L^{\alpha \beta \alpha^{\prime}} L_{\alpha \beta \beta^{\prime}} \Delta H^{\beta^{\prime}}+|\nabla H|^{2} H^{\alpha^{\prime}} \\
& +2 \Delta\left(L_{\alpha \beta}^{\alpha^{\prime}} L_{\beta^{\prime}}^{\alpha \beta} H^{\beta^{\prime}}\right)+2 \nabla_{\alpha} \nabla_{\beta}\left(L^{\alpha \beta \beta^{\prime}} H_{\beta^{\prime}} H^{\alpha^{\prime}}\right) \\
& +2 L_{\gamma \delta}^{\alpha^{\prime}} L_{\beta^{\prime}}^{\gamma \delta} L_{\alpha \beta \gamma^{\prime}} L^{\alpha \beta \beta^{\prime}} H^{\gamma^{\prime}}+2 L_{\alpha \gamma}^{\alpha^{\prime}} L_{\beta \beta^{\prime}}^{\gamma} L^{\alpha \beta \gamma^{\prime}} H^{\beta^{\prime}} H_{\gamma^{\prime}}-L_{\alpha \beta}^{\gamma^{\prime}} L_{\beta^{\prime}}^{\alpha \beta} H_{\gamma^{\prime}} H^{\beta^{\prime}} H^{\alpha^{\prime}} \\
& -\frac{7}{4} \Delta\left(|H|^{2} H^{\alpha^{\prime}}\right)-\frac{7}{4} L_{\alpha \beta}^{\alpha^{\prime}} L_{\beta^{\prime}}^{\alpha \beta}|H|^{2} H^{\beta^{\prime}}+\frac{7}{16}|H|^{4} H^{\alpha^{\prime}} .
\end{aligned}
$$

We checked this formula by evaluating it on the anchor ring embeddings $T_{R, r}^{2,2}$ and $T_{R, r}^{3,1}$ discussed in 96.2 . It vanishes exactly for the values of $R$ and $r$ corresponding to the $\mathcal{E}$-critical anchor ring embeddings described there.

We close this section by noting that modulo linear terms in background curvature and quadratic terms in derivatives of $g$, we have

$$
\begin{aligned}
(2 l) ! U_{(2 l)}^{\alpha^{\prime}} & =\frac{1 \cdot 3 \cdot 5 \cdots(2 l-1)}{k(k-2) \cdots(k+2-2 l)} \Delta^{l-1} H^{\alpha^{\prime}}, \quad 1 \leq l \leq k / 2 \\
k ! \mathcal{H}^{\alpha^{\prime}} & =\frac{1 \cdot 3 \cdot 5 \cdots(k-1)}{k(k-2) \cdots 2} \Delta^{k / 2} H^{\alpha^{\prime}}, \quad k \geq 2
\end{aligned}
$$

These follow by induction keeping track only of the linear terms in the calculations indicated above. Modulo terms involving background curvature and terms of higher homogeneity degree in the second fundamental form and its derivatives, the energy has the form

$$
\mathcal{E}=c_{k} \int_{\Sigma}\left\langle H, \Delta^{(k-2) / 2} H\right\rangle d a_{\Sigma}, \quad k \geq 2, \quad c_{k} \neq 0
$$




\section{Dimension Four}

In this section we take $k=4$ throughout (so $n \geq 5$ ), and $M$ will be either $\mathbb{R}^{n}$ with the Euclidean metric or $S^{n}$ with the round metric of sectional curvature $1 . \quad \Sigma$ is a compact 4-dimensional immersed submanifold of $M$.

If $M=\mathbb{R}^{n}$, then all background curvature vanishes, so the formula in Corollary 5.3 for the $k=4$ energy becomes (1.1). If $M=S^{n}$, then the Weyl, Cotton and Bach tensors all vanish, and the Schouten tensor is given by $P=\frac{1}{2} g$. In this case the formula in Corollary 5.3 reduces to $(1.2)$. Set $\underline{\mathcal{E}}=128 \mathcal{E}$.

Proposition 4.5 shows that if $\Sigma$ is a 4-dimensional immersed submanifold of either $\mathbb{R}^{n}$ or $S^{n}$ and $\Sigma$ is minimal, then $\Sigma$ is critical for $\mathcal{E}$. As noted in the introduction, this also follows immediately from (1.1) and (1.2), since all terms are quadratic in $H$ except for the constant term in (1.2), which corresponds to a multiple of the area of $\Sigma$. Of course there are no compact minimal submanifolds of $\mathbb{R}^{n}$. But the statement holds also for noncompact minimal submanifolds in the sense that the Euler-Lagrange equation for $\mathcal{E}$ holds, corresponding to compactly supported variations. Note that in the case that $\Sigma \subset S^{n}$ is minimal, we have $\underline{\mathcal{E}}(\Sigma)=48 \operatorname{Area}(\Sigma)$

6.1. Products of Spheres. Proposition 4.5 provides many examples of $\mathcal{E}$-critical submanifolds. Just as for the classical $k=2$ Willmore energy, minimal submanifolds of $S^{n}$ or their images in $\mathbb{R}^{n}$ under stereographic projection are $\mathcal{E}$-critical. So a totally geodesic $S^{4} \subset S^{n}$, or any round $S^{4} \subset \mathbb{R}^{n}$, is $\mathcal{E}$-critical. Likewise, the standard examples of minimal embeddings of 4-dimensional products of spheres in $S^{n}$ are $\mathcal{E}$-critical. These are:

$$
\begin{array}{rlrl}
S^{2}\left(\frac{1}{\sqrt{2}}\right) \times S^{2}\left(\frac{1}{\sqrt{2}}\right) \subset S^{5} & & \underline{\mathcal{E}}=192 \pi^{2} \\
S^{1}\left(\frac{1}{2}\right) \times S^{3}\left(\frac{\sqrt{3}}{2}\right) \subset S^{5} & & \underline{\mathcal{E}}=36 \sqrt{3} \pi^{3} \\
S^{1}\left(\frac{1}{2}\right) \times S^{1}\left(\frac{1}{2}\right) \times S^{2}\left(\frac{1}{\sqrt{2}}\right) \subset S^{6} & \underline{\mathcal{E}}=96 \pi^{3} \\
S^{1}\left(\frac{1}{2}\right) \times S^{1}\left(\frac{1}{2}\right) \times S^{1}\left(\frac{1}{2}\right) \times S^{1}\left(\frac{1}{2}\right) \subset S^{7} & \underline{\mathcal{E}}=48 \pi^{4}
\end{array}
$$

For each of the four topologies appearing in (6.1), there is a family of embeddings generalizing (6.1) obtained by varying the radii of the factor spheres. Namely, we have

$$
\begin{gathered}
S^{2}\left(r_{1}\right) \times S^{2}\left(r_{2}\right) \subset S^{5} \\
S^{1}\left(r_{1}\right) \times S^{3}\left(r_{2}\right) \subset S^{5} \\
S^{1}\left(r_{1}\right) \times S^{1}\left(r_{2}\right) \times S^{2}\left(r_{3}\right) \subset S^{6} \\
S^{1}\left(r_{1}\right) \times S^{1}\left(r_{2}\right) \times S^{1}\left(r_{3}\right) \times S^{1}\left(r_{4}\right) \subset S^{7},
\end{gathered}
$$

where in each case $\sum r_{k}^{2}=1$. In this section we calculate explicitly the energy of these embeddings as a function of the $r_{k}$. We deduce two consequences. One consequence is that in the three families other than $S^{2} \times S^{2} \subset S^{5}, \mathcal{E}$ is already unbounded above and below when restricted to the family. This will prove Proposition 1.1 in these cases. The other consequence is that we identify a non-minimal critical point of $\mathcal{E}$ in each of the three families other than $S^{2} \times S^{2}$. Specifically, we prove 
Proposition 6.1. In addition to (6.1), the following are critical for $\mathcal{E}$ :

$$
\begin{aligned}
S^{1}\left(\sqrt{\frac{3}{8}}\right) \times S^{3}\left(\sqrt{\frac{5}{8}}\right) \subset S^{5} & \underline{\mathcal{E}}=16 \sqrt{15} \pi^{3} \\
S^{1}\left(\sqrt{\frac{5}{24}}\right) \times S^{1}\left(\sqrt{\frac{9}{24}}\right) \times S^{2}\left(\sqrt{\frac{10}{24}}\right) \subset S^{6} & \underline{\mathcal{E}}=\frac{128 \sqrt{5}}{3} \pi^{3} \\
S^{1}\left(\sqrt{\frac{5}{24}}\right) \times S^{1}\left(\sqrt{\frac{5}{24}}\right) \times S^{1}\left(\sqrt{\frac{5}{24}}\right) \times S^{1}\left(\sqrt{\frac{9}{24}}\right) \subset S^{7} & \underline{\mathcal{E}}=\frac{64 \sqrt{5}}{3} \pi^{4}
\end{aligned}
$$

Zhang $([\mathrm{Z}])$ asserts that (6.1) together with (6.3) constitute all $\mathcal{E}$-critical embeddings in the families (6.2).

Consider first $S^{2}\left(r_{1}\right) \times S^{2}\left(r_{2}\right) \subset S^{5}$. Parametrize $S^{2}\left(r_{1}\right) \subset \mathbb{R}^{3}$ using spherical coordinates:

$$
y_{1}=r_{1}\left(\sin \phi_{1} \cos \theta_{1}, \sin \phi_{1} \sin \theta_{1}, \cos \phi_{1}\right)
$$

and likewise $S^{2}\left(r_{2}\right) \subset \mathbb{R}^{3}$ :

$$
y_{2}=r_{2}\left(\sin \phi_{2} \cos \theta_{2}, \sin \phi_{2} \sin \theta_{2}, \cos \phi_{2}\right)
$$

where $0 \leq \theta_{1}, \theta_{2}<2 \pi, 0 \leq \phi_{1}, \phi_{2} \leq \pi$. Then $x=\left(y_{1}, y_{2}\right)$ is our embedding $S^{2}\left(r_{1}\right) \times S^{2}\left(r_{2}\right) \subset$ $S^{5}(1) \subset \mathbb{R}^{6}$.

The following is an orthonormal basis for the tangent space to $S^{2}\left(r_{1}\right) \times S^{2}\left(r_{2}\right)$ :

$$
\begin{aligned}
e_{1} & =\frac{x_{\phi_{1}}}{\left|x_{\phi_{1}}\right|}=\frac{1}{r_{1}} x_{\phi_{1}} \\
& =\left(\cos \phi_{1} \cos \theta_{1}, \cos \phi_{1} \sin \theta_{1},-\sin \phi_{1}, 0,0,0\right) \\
e_{2} & =\frac{x_{\theta_{1}}}{\left|x_{\theta_{1}}\right|}=\frac{1}{r_{1} \sin \phi_{1}} x_{\theta_{1}} \\
& =\left(-\sin \theta_{1}, \cos \theta_{1}, 0,0,0,0\right) \\
e_{3} & =\frac{x_{\phi_{2}}}{\left|x_{\phi_{2}}\right|}=\frac{1}{r_{2}} x_{\phi_{2}} \\
& =\left(0,0,0, \cos \phi_{2} \cos \theta_{2}, \cos \phi_{2} \sin \theta_{2},-\sin \phi_{2}\right) \\
e_{4} & =\frac{x_{\theta_{2}}}{\left|x_{\theta_{2}}\right|}=\frac{1}{r_{2} \sin \phi_{2}} x_{\theta_{2}} \\
& =\left(0,0,0,-\sin \theta_{2}, \cos \theta_{2}, 0\right) .
\end{aligned}
$$

Set

$$
\nu=\left(-\frac{r_{2}}{r_{1}} y_{1}, \frac{r_{1}}{r_{2}} y_{2}\right) .
$$

It is easily checked that $e_{1}, e_{2}, e_{3}, e_{4}, \nu$ is an orthonormal basis for the tangent space to $S^{5}$ along $S^{2}\left(r_{1}\right) \times S^{2}\left(r_{2}\right)$. In the subsequent discussion in which a unit normal has been chosen for a particular hypersurface embedding, we identify $L$ and $H$ with their scalar counterparts determined by the chosen normal. The second fundamental form in this basis is thus given by

$$
L_{\alpha \beta}=\left\langle\nabla_{e_{\alpha}} e_{\beta}, \nu\right\rangle
$$

Now

$$
\nabla_{e_{\alpha}} e_{\beta}=\nabla_{\frac{\partial_{\alpha} x}{\left|\partial_{\alpha} x\right|}} e_{\beta}=\frac{1}{\left|\partial_{\alpha} x\right|} \partial_{\alpha} e_{\beta},
$$


where $\partial_{1}=\partial_{\phi_{1}}, \partial_{2}=\partial_{\theta_{1}}, \partial_{3}=\partial_{\phi_{2}}, \partial_{4}=\partial_{\theta_{2}}$. From the expressions given above for $e_{\alpha}$, it is easy to see that $\nabla_{e_{\alpha}} e_{\beta}=0$ except for the following cases:

$$
\begin{aligned}
\nabla_{e_{1}} e_{1} & =\frac{1}{r_{1}}\left(-\sin \phi_{1} \cos \theta_{1},-\sin \phi_{1} \sin \theta_{1},-\cos \phi_{1}, 0,0,0\right) \\
\nabla_{e_{2}} e_{1} & =\frac{1}{r_{1} \sin \phi_{1}}\left(-\cos \phi_{1} \sin \theta_{1}, \cos \phi_{1} \cos \theta_{1}, 0,0,0,0\right) \\
\nabla_{e_{2}} e_{2} & =\frac{1}{r_{1} \sin \phi_{1}}\left(-\cos \theta_{1},-\sin \theta_{1}, 0,0,0,0\right) \\
\nabla_{e_{3}} e_{3} & =\frac{1}{r_{2}}\left(0,0,0,-\sin \phi_{2} \cos \theta_{2},-\sin \phi_{2} \sin \theta_{2},-\cos \phi_{2}\right) \\
\nabla_{e_{4}} e_{3} & =\frac{1}{r_{2} \sin \phi_{2}}\left(0,0,0,-\cos \phi_{2} \sin \theta_{2}, \cos \phi_{2} \cos \theta_{2}, 0\right) \\
\nabla_{e_{4}} e_{4} & =\frac{1}{r_{2} \sin \phi_{2}}\left(0,0,0,-\cos \theta_{2},-\sin \theta_{2}, 0\right) .
\end{aligned}
$$

Therefore, in this basis,

$$
L_{\alpha \beta}=\left(\begin{array}{cccc}
\frac{r_{2}}{r_{1}} & 0 & 0 & 0 \\
0 & \frac{r_{2}}{r_{1}} & 0 & 0 \\
0 & 0 & -\frac{r_{1}}{r_{2}} & 0 \\
0 & 0 & 0 & -\frac{r_{1}}{r_{2}}
\end{array}\right)
$$

Thus

$$
H=2\left(\frac{r_{2}}{r_{1}}-\frac{r_{1}}{r_{2}}\right), \quad|L|^{2}=2\left(\frac{r_{1}^{2}}{r_{2}^{2}}+\frac{r_{2}^{2}}{r_{1}^{2}}\right) .
$$

Substituting into (1.2) and simplifying using $r_{1}^{2}+r_{2}^{2}=1$ gives

$$
\begin{aligned}
\underline{\mathcal{E}} & =-16 \pi^{2} \frac{r_{1}^{4}+r_{2}^{4}-14 r_{1}^{2} r_{2}^{2}}{r_{1}^{2} r_{2}^{2}} \\
& =-16 \pi^{2}\left(t^{2}+t^{-2}-14\right)
\end{aligned}
$$

with $\frac{r_{1}}{r_{2}}=t$. The function $t^{2}+t^{-2}$ of $t \in(0, \infty)$ has a unique critical point at $t=1$, a global minimum. Thus $\underline{\mathcal{E}}$ is unbounded below on this family, with a maximum of $192 \pi^{2}$ achieved at the minimal embedding. We remark that since the formula for $\underline{\mathcal{E}}$ above is homogeneous of degree 0 in $\left(r_{1}, r_{2}\right)$, it is valid by conformal invariance for the embedding $S^{2}\left(r_{1}\right) \times S^{2}\left(r_{2}\right) \subset$ $S^{5}\left(\sqrt{r_{1}^{2}+r_{2}^{2}}\right)$ for all $r_{1}, r_{2}>0$.

The calculations for the other families in (6.2) are similar. We briefly outline the computations in each case.

For $S^{1} \times S^{3}$, parametrize $S^{1}\left(r_{1}\right) \subset \mathbb{R}^{2}$ as

$$
y_{1}=r_{1}\left(\cos \theta_{1}, \sin \theta_{1}\right)
$$

and $S^{3}\left(r_{2}\right) \subset \mathbb{R}^{4}$ as

$$
y_{2}=r_{2}\left(\sin \phi_{1} \sin \phi_{2} \sin \theta_{2}, \sin \phi_{1} \sin \phi_{2} \cos \theta_{2}, \sin \phi_{1} \cos \phi_{2}, \cos \phi_{1}\right) \text {. }
$$


Then $x=\left(y_{1}, y_{2}\right)$ is our embedding $S^{1}\left(r_{1}\right) \times S^{3}\left(r_{2}\right) \subset S^{5}(1) \subset \mathbb{R}^{6}$. The vectors

$$
e_{1}=\frac{x_{\theta_{1}}}{\left|x_{\theta_{1}}\right|}, \quad e_{2}=\frac{x_{\phi_{1}}}{\left|x_{\phi_{1}}\right|}, \quad e_{3}=\frac{x_{\phi_{2}}}{\left|x_{\phi_{2}}\right|}, \quad e_{4}=\frac{x_{\theta_{2}}}{\left|x_{\theta_{2}}\right|}, \quad \nu=\left(-\frac{r_{2}}{r_{1}} y_{1}, \frac{r_{1}}{r_{2}} y_{2}\right)
$$

form an orthonormal basis for the tangent space to $S^{5}$ along $S^{1}\left(r_{1}\right) \times S^{3}\left(r_{2}\right)$. The second fundamental form (6.5) in this basis now takes the form

$$
L_{\alpha \beta}=\left(\begin{array}{cccc}
\frac{r_{2}}{r_{1}} & 0 & 0 & 0 \\
0 & -\frac{r_{1}}{r_{2}} & 0 & 0 \\
0 & 0 & -\frac{r_{1}}{r_{2}} & 0 \\
0 & 0 & 0 & -\frac{r_{1}}{r_{2}}
\end{array}\right) .
$$

So

$$
H=\frac{r_{2}}{r_{1}}-3 \frac{r_{1}}{r_{2}}, \quad|L|^{2}=\frac{r_{2}^{2}}{r_{1}^{2}}+3 \frac{r_{1}^{2}}{r_{2}^{2}} .
$$

This time substituting into (1.2) and simplifying using $r_{1}^{2}+r_{2}^{2}=1$ gives

$$
\begin{aligned}
\underline{\mathcal{E}} & =\frac{9 \pi^{3}}{4} \frac{15 r_{1}^{4} r_{2}^{2}+14 r_{1}^{2} r_{2}^{4}-r_{2}^{6}}{r_{1}^{3} r_{2}^{3}} \\
& =\frac{9 \pi^{3}}{4} \frac{15 t^{4}+14 t^{2}-1}{t^{3}}
\end{aligned}
$$

with $t=\frac{r_{1}}{r_{2}}$. This function of $t$ goes to $+\infty$ as $t \rightarrow \infty$ and goes to $-\infty$ as $t \rightarrow 0$. So it is unbounded above and below. It has two critical points, a local maximum at $t=\frac{1}{\sqrt{3}}$, corresponding to the minimal embedding, and a local minimum at $t=\sqrt{\frac{3}{5}}$. The Principle of Symmetric Criticality $([\mathrm{P})$, or alternately Hsiang's argument $[\mathrm{H}]$ concerning critical orbits of compact groups of isometries, which applies equally well to $\mathcal{E}$ as to the area functional, implies that $S^{1}\left(\sqrt{\frac{3}{8}}\right) \times S^{3}\left(\sqrt{\frac{5}{8}}\right) \subset S^{5}$ is critical for $\mathcal{E}$. This is the first example in (6.3).

For $S^{1} \times S^{1} \times S^{2}$, parametrize $S^{1}\left(r_{1}\right) \subset \mathbb{R}^{2}$ and $S^{1}\left(r_{2}\right) \subset \mathbb{R}^{2}$ by

$$
y_{1}=r_{1}\left(\cos \theta_{1}, \sin \theta_{1}\right), \quad y_{2}=r_{2}\left(\cos \theta_{2}, \sin \theta_{2}\right)
$$

and $S^{2}\left(r_{3}\right) \subset \mathbb{R}^{3}$ by

$$
y_{3}=r_{3}\left(\sin \phi \cos \theta_{3}, \sin \phi \sin \theta_{3}, \cos \phi\right) .
$$

Then $x=\left(y_{1}, y_{2}, y_{3}\right)$ is our embedding $S^{1}\left(r_{1}\right) \times S^{1}\left(r_{2}\right) \times S^{2}\left(r_{3}\right) \subset S^{6}(1) \subset \mathbb{R}^{7}$. The vectors

$$
e_{1}=\frac{x_{\theta_{1}}}{\left|x_{\theta_{1}}\right|}, \quad e_{2}=\frac{x_{\theta_{2}}}{\left|x_{\theta_{2}}\right|}, \quad e_{3}=\frac{x_{\theta_{3}}}{\left|x_{\theta_{3}}\right|}, \quad e_{4}=\frac{x_{\phi}}{\left|x_{\phi}\right|}
$$

form an orthonormal basis for the tangent space to $S^{1}\left(r_{1}\right) \times S^{1}\left(r_{2}\right) \times S^{2}\left(r_{3}\right)$. The vectors

$$
\begin{aligned}
& \nu_{1}=\left(-\frac{r_{2}}{r_{1} \sqrt{r_{1}^{2}+r_{2}^{2}}} y_{1}, \frac{r_{1}}{r_{2} \sqrt{r_{1}^{2}+r_{2}^{2}}} y_{2}, 0\right) \\
& \nu_{2}=\left(\frac{r_{3}}{\sqrt{r_{1}^{2}+r_{2}^{2}}} y_{1}, \frac{r_{3}}{\sqrt{r_{1}^{2}+r_{2}^{2}}} y_{2},-\frac{\sqrt{r_{1}^{2}+r_{2}^{2}}}{r_{3}} y_{3}\right)
\end{aligned}
$$


form an orthonormal basis for the normal space. The second fundamental forms $L_{\alpha \beta}^{\alpha^{\prime}}=$ $\left\langle\nabla_{e_{\alpha}} e_{\beta}, \nu_{\alpha^{\prime}}\right\rangle, \alpha^{\prime}=1,2$, are given by

$L_{\alpha \beta}^{1}=\left(\begin{array}{cccc}\frac{r_{2}}{r_{1} \sqrt{r_{1}^{2}+r_{2}^{2}}} & 0 & 0 & 0 \\ 0 & -\frac{r_{1}}{r_{2} \sqrt{r_{1}^{2}+r_{2}^{2}}} & 0 & 0 \\ 0 & 0 & 0 & 0 \\ 0 & 0 & 0 & 0\end{array}\right), L_{\alpha \beta}^{2}=\left(\begin{array}{cccc}-\frac{r_{3}}{\sqrt{r_{1}^{2}+r_{2}^{2}}} & 0 & 0 & 0 \\ 0 & -\frac{r_{3}}{\sqrt{r_{1}^{2}+r_{2}^{2}}} & 0 & 0 \\ 0 & 0 & \frac{\sqrt{r_{1}^{2}+r_{2}^{2}}}{r_{3}} & 0 \\ 0 & 0 & 0 & \frac{\sqrt{r_{1}^{2}+r_{2}^{2}}}{r_{3}}\end{array}\right)$

and the mean curvatures by

$$
H^{1}=\frac{r_{2}^{2}-r_{1}^{2}}{r_{1} r_{2} \sqrt{r_{1}^{2}+r_{2}^{2}}}, \quad H^{2}=\frac{2\left(r_{1}^{2}+r_{2}^{2}-r_{3}^{2}\right)}{r_{3} \sqrt{r_{1}^{2}+r_{2}^{2}}} .
$$

We used Mathematica and $r_{1}^{2}+r_{2}^{2}+r_{3}^{2}=1$ to calculate that

$$
\begin{aligned}
\underline{\mathcal{E}} & =-\frac{\pi^{3}}{r_{1}^{3} r_{2}^{3} r_{3}^{3}}\left[16 r_{1}^{4} r_{2}^{4} r_{3}-56\left(r_{1}^{4} r_{2}^{2}+r_{2}^{4} r_{1}^{2}\right) r_{3}^{3}+\left(9\left(r_{1}^{4}+r_{2}^{4}\right)-14 r_{1}^{2} r_{2}^{2}\right) r_{3}^{5}\right] \\
& =-\frac{\pi^{3}}{t_{1}^{3} t_{2}^{3}}\left[16 t_{1}^{4} t_{2}^{4}-56\left(t_{1}^{4} t_{2}^{2}+t_{2}^{4} t_{1}^{2}\right)+9\left(t_{1}^{4}+t_{2}^{4}\right)-14 t_{1}^{2} t_{2}^{2}\right]
\end{aligned}
$$

with $t_{1}=\frac{r_{1}}{r_{3}}, t_{2}=\frac{r_{2}}{r_{3}}$. It is easily seen that this function of $\left(t_{1}, t_{2}\right) \in(0, \infty) \times(0, \infty)$ is unbounded above and below (for instance, this is already the case when restricted to $t_{2}=1$ ) and has critical points at $\left(t_{1}, t_{2}\right)=\left(\frac{1}{\sqrt{2}}, \frac{1}{\sqrt{2}}\right)$ and $\left(t_{1}, t_{2}\right)=\left(\sqrt{\frac{5}{10}}, \sqrt{\frac{9}{10}}\right)$. (By symmetry, another critical point is obtained from the latter by interchanging $t_{1}$ and $t_{2}$.) The first critical point corresponds to the minimal embedding $r_{1}=r_{2}=\frac{1}{2}, r_{3}=\frac{1}{\sqrt{2}}$. The second corresponds to the non-minimal $\mathcal{E}$-critical embedding $S^{1}\left(\sqrt{\frac{5}{24}}\right) \times S^{1}\left(\sqrt{\frac{9}{24}}\right) \times S^{2}\left(\sqrt{\frac{10}{24}}\right) \subset S^{6}$. This is the second example in (6.3).

For $\left(S^{1}\right)^{4}$, parametrize $S^{1}\left(r_{\alpha}\right) \subset \mathbb{R}^{2}, 1 \leq \alpha \leq 4$, by

$$
y_{\alpha}=r_{\alpha}\left(\cos \theta_{\alpha}, \sin \theta_{\alpha}\right) \text {. }
$$

Then $x=\left(y_{1}, y_{2}, y_{3}, y_{4}\right)$ is our embedding $S^{1}\left(r_{1}\right) \times S^{1}\left(r_{2}\right) \times S^{1}\left(r_{3}\right) \times S^{1}\left(r_{4}\right) \subset S^{7}(1) \subset \mathbb{R}^{8}$. The vectors

$$
e_{\alpha}=\frac{x_{\theta_{\alpha}}}{r_{\alpha}}, \quad 1 \leq \alpha \leq 4
$$

form an orthonormal basis for the tangent space to $S^{1}\left(r_{1}\right) \times S^{1}\left(r_{2}\right) \times S^{1}\left(r_{3}\right) \times S^{1}\left(r_{4}\right)$. The vectors

$$
\begin{aligned}
\nu_{1} & =\left(-\frac{r_{2}}{r_{1}} y_{1}, \frac{r_{1}}{r_{2}} y_{2},-\frac{r_{4}}{r_{3}} y_{3}, \frac{r_{3}}{r_{4}} y_{4}\right) \\
\nu_{2} & =\left(-\frac{r_{3}}{r_{1}} y_{1}, \frac{r_{4}}{r_{2}} y_{2}, \frac{r_{1}}{r_{3}} y_{3},-\frac{r_{2}}{r_{4}} y_{4}\right) \\
\nu_{3} & =\left(-\frac{r_{4}}{r_{1}} y_{1},-\frac{r_{3}}{r_{2}} y_{2}, \frac{r_{2}}{r_{3}} y_{3}, \frac{r_{1}}{r_{4}} y_{4}\right)
\end{aligned}
$$


form an orthonormal basis for the normal space. The second fundamental forms $L_{\alpha \beta}^{\alpha^{\prime}}=$ $\left\langle\nabla_{e_{\alpha}} e_{\beta}, \nu_{\alpha^{\prime}}\right\rangle$ are given by

$$
L_{\alpha \beta}^{1}=\left(\begin{array}{cccc}
\frac{r_{2}}{r_{1}} & 0 & 0 & 0 \\
0 & -\frac{r_{1}}{r_{2}} & 0 & 0 \\
0 & 0 & \frac{r_{4}}{r_{3}} & 0 \\
0 & 0 & 0 & -\frac{r_{3}}{r_{4}}
\end{array}\right), L_{\alpha \beta}^{2}=\left(\begin{array}{cccc}
\frac{r_{3}}{r_{1}} & 0 & 0 & 0 \\
0 & -\frac{r_{4}}{r_{2}} & 0 & 0 \\
0 & 0 & -\frac{r_{1}}{r_{3}} & 0 \\
0 & 0 & 0 & \frac{r_{2}}{r_{4}}
\end{array}\right), L_{\alpha \beta}^{3}=\left(\begin{array}{cccc}
\frac{r_{4}}{r_{1}} & 0 & 0 & 0 \\
0 & \frac{r_{3}}{r_{2}} & 0 & 0 \\
0 & 0 & -\frac{r_{2}}{r_{3}} & 0 \\
0 & 0 & 0 & -\frac{r_{1}}{r_{4}}
\end{array}\right)
$$

and the mean curvatures by

$$
H^{1}=\frac{r_{2}^{2}-r_{1}^{2}}{r_{1} r_{2}}+\frac{r_{4}^{2}-r_{3}^{2}}{r_{3} r_{4}}, \quad H^{2}=\frac{r_{3}^{2}-r_{1}^{2}}{r_{1} r_{3}}+\frac{r_{2}^{2}-r_{4}^{2}}{r_{2} r_{4}}, \quad H^{3}=\frac{r_{4}^{2}-r_{1}^{2}}{r_{1} r_{4}}+\frac{r_{3}^{2}-r_{2}^{2}}{r_{2} r_{3}} .
$$

We used Mathematica and $\sum_{\alpha=1}^{4} r_{\alpha}^{2}=1$ to calculate that

$$
\begin{aligned}
\underline{\mathcal{E}} & =-\frac{\pi^{4}}{r_{1}^{3} r_{2}^{3} r_{3}^{3} r_{4}^{3}}\left[36 \operatorname{Sym}\left(r_{1}^{4} r_{2}^{4} r_{3}^{4}\right)-84 \operatorname{Sym}\left(r_{1}^{4} r_{2}^{4} r_{3}^{2} r_{4}^{2}\right)\right] \\
& =-\frac{\pi^{4}}{t_{1}^{3} t_{2}^{3} t_{3}^{3}}\left[9 t_{1}^{4} t_{2}^{4} t_{3}^{4}+27 \operatorname{Sym}\left(t_{1}^{4} t_{2}^{4}\right)-42 \operatorname{Sym}\left(t_{1}^{4} t_{2}^{4} t_{3}^{2}\right)-42 \operatorname{Sym}\left(t_{1}^{4} t_{2}^{2} t_{3}^{2}\right)\right]
\end{aligned}
$$

where $t_{k}=\frac{r_{k}}{r_{4}}, 1 \leq k \leq 3$. Here we write

$$
\begin{aligned}
\operatorname{Sym}\left(r_{1}^{a} r_{2}^{b} r_{3}^{c} r_{4}^{d}\right) & =\frac{1}{4 !} \sum_{\sigma \in S_{4}} r_{\sigma(1)}^{a} r_{\sigma(2)}^{b} r_{\sigma(3)}^{c} r_{\sigma(4)}^{d} \\
\operatorname{Sym}\left(t_{1}^{a} t_{2}^{b} t_{3}^{c}\right) & =\frac{1}{3 !} \sum_{\sigma \in S_{3}} t_{\sigma(1)}^{a} t_{\sigma(2)}^{b} t_{\sigma(3)}^{c}
\end{aligned}
$$

for the symmetrization of monomials. It is easily seen that $\underline{\mathcal{E}}$, viewed as a function of $\left(t_{1}, t_{2}, t_{3}\right) \in(0, \infty)^{3}$, is unbounded above and below (for instance, this is already the case when restricted to $\left.t_{2}=t_{3}=1\right)$ and has critical points at $\left(t_{1}, t_{2}, t_{3}\right)=(1,1,1)$ and $\left(t_{1}, t_{2}, t_{3}\right)=$ $\left(\sqrt{\frac{5}{9}}, \sqrt{\frac{5}{9}}, \sqrt{\frac{5}{9}}\right)$. The first critical point corresponds to the minimal embedding $r_{1}=r_{2}=$ $r_{3}=r_{4}=\frac{1}{2}$. The second corresponds to the non-minimal $\mathcal{E}$-critical embedding $S^{1}\left(\sqrt{\frac{5}{24}}\right) \times$ $S^{1}\left(\sqrt{\frac{5}{24}}\right) \times S^{1}\left(\sqrt{\frac{5}{24}}\right) \times S^{1}\left(\sqrt{\frac{9}{24}}\right) \subset S^{7}$. This is the third example in (6.3).

We close our discussion of the energy of the embeddings (6.2) with an observation that we find truly remarkable. Namely, the energy and critical points for the first three of the families in (6.2), as well as for equatorial $S^{4} \subset S^{5}$, can all be derived from the energy and critical points for the 4-torus family $S^{1}\left(r_{1}\right) \times S^{1}\left(r_{2}\right) \times S^{1}\left(r_{3}\right) \times S^{1}\left(r_{4}\right) \subset S^{7}$ by specializing 
the radii. Precisely, the following relations hold:

$$
\begin{aligned}
& \mathcal{E}\left(S^{1}\left(r_{1}\right) \times S^{1}\left(r_{2}\right) \times S^{1}\left(r_{3}\right) \times S^{1}\left(r_{3}\right)\right)=\frac{\pi}{2} \mathcal{E}\left(S^{1}\left(r_{1}\right) \times S^{1}\left(r_{2}\right) \times S^{2}\left(\sqrt{2} r_{3}\right)\right) \\
& \mathcal{E}\left(S^{1}\left(r_{1}\right) \times S^{1}\left(r_{2}\right) \times S^{1}\left(r_{2}\right) \times S^{1}\left(r_{2}\right)\right)=\frac{4 \pi}{3 \sqrt{3}} \mathcal{E}\left(S^{1}\left(r_{1}\right) \times S^{3}\left(\sqrt{3} r_{2}\right)\right) \\
& \mathcal{E}\left(S^{1}\left(r_{1}\right) \times S^{1}\left(r_{1}\right) \times S^{1}\left(r_{2}\right) \times S^{1}\left(r_{2}\right)\right)=\frac{\pi^{2}}{4} \mathcal{E}\left(S^{2}\left(\sqrt{2} r_{1}\right) \times S^{2}\left(\sqrt{2} r_{2}\right)\right) \\
& \mathcal{E}\left(S^{1}\left(r_{1}\right) \times S^{1}\left(r_{1}\right) \times S^{1}\left(r_{1}\right) \times S^{1}\left(r_{1}\right)\right)=\frac{3 \pi^{2}}{8} \mathcal{E}\left(S^{4}\left(2 r_{1}\right)\right)
\end{aligned}
$$

Moreover, the constants appearing in these relations are the corresponding ratios of the areas of the factor spheres. Namely,

$$
\begin{aligned}
\frac{\pi}{2} & =\frac{A\left(S^{1}\left(r_{1}\right)\right) A\left(S^{1}\left(r_{2}\right)\right) A\left(S^{1}\left(r_{3}\right)\right)^{2}}{A\left(S^{1}\left(r_{1}\right)\right) A\left(S^{1}\left(r_{2}\right)\right) A\left(S^{2}\left(\sqrt{2} r_{3}\right)\right)} \\
\frac{4 \pi}{3 \sqrt{3}} & =\frac{A\left(S^{1}\left(r_{1}\right)\right) A\left(S^{1}\left(r_{2}\right)\right)^{3}}{A\left(S^{1}\left(r_{1}\right)\right) A\left(S^{3}\left(\sqrt{3} r_{2}\right)\right)} \\
\frac{\pi^{2}}{4} & =\frac{A\left(S^{1}\left(r_{1}\right)\right)^{2} A\left(S^{1}\left(r_{2}\right)\right)^{2}}{A\left(S^{2}\left(\sqrt{2} r_{1}\right)\right) A\left(S^{2}\left(\sqrt{2} r_{2}\right)\right)} \\
\frac{3 \pi^{2}}{8} & =\frac{A\left(S^{1}\left(r_{1}\right)\right)^{4}}{A\left(S^{4}\left(2 r_{1}\right)\right)} .
\end{aligned}
$$

It is clear that these relations enable the deduction of the critical points of any of the families from those for $S^{1}\left(r_{1}\right) \times S^{1}\left(r_{2}\right) \times S^{1}\left(r_{3}\right) \times S^{1}\left(r_{4}\right)$. Also, one may deduce direct relations between the energy of any one of the families and any other family with fewer factors. That is, the energy of the family $S^{1}\left(r_{1}\right) \times S^{1}\left(r_{2}\right) \times S^{2}\left(r_{3}\right)$ determines the energy of the $S^{1} \times S^{3}, S^{2} \times S^{2}$, and $S^{4}$ families, and the energy of either family $S^{1} \times S^{3}$ or $S^{2} \times S^{2}$ determines that of the $S^{4}$ family, by similar relations.

We also note that the same sort of relation holds for the usual 2-dimensional Willmore energy, with the same rule for the constant. Namely, for the 2-dimensional energy $\mathcal{E}$ given in Corollary 5.3, we have

$$
\mathcal{E}\left(S^{1}\left(r_{1}\right) \times S^{1}\left(r_{1}\right)\right)=\frac{\pi}{2} \mathcal{E}\left(S^{2}\left(\sqrt{2} r_{1}\right)\right)
$$

with $\frac{\pi}{2}=\frac{A\left(S^{1}\left(r_{1}\right)\right)^{2}}{A\left(S^{2}\left(\sqrt{2} r_{1}\right)\right)}$. (No such relation seems to hold for the Willmore energy in the form $\int_{\Sigma}|\stackrel{\circ}{L}|^{2}$ owing to the extra term involving the Euler characteristic.) We have no understanding of why these relations should be true other than verifying them from the formulas. It would be interesting to provide a geometric explanation.

6.2. Anchor Rings. We first indicate how the embeddings $S^{j} \times S^{k} \subset S^{j+k+1}$ considered in 6.1 can be composed with stereographic projection to obtain embeddings $S^{j} \times S^{k} \subset$ $\mathbb{R}^{j+k+1}$ which are higher dimensional versions of anchor rings. We then construct a family of embeddings of $S^{2} \times S^{2}$ in $\mathbb{R}^{5}$ for which $\mathcal{E} \rightarrow \infty$ by dilating such an anchor ring in only some of the variables. This combined with the results in $\$ 6.1$ enables us to prove Proposition [1.1. 
Fix $R>0$ and $0<r<R$. Define the anchor ring embedding $S^{j} \times S^{k} \rightarrow \mathbb{R}^{j+k+1}$ by

$$
S^{j} \times S^{k} \ni(y, z) \rightarrow((R+r w) y, r v)
$$

where we have written $z=(v, w) \in S^{k} \subset \mathbb{R}^{k+1}$ with $v \in \mathbb{R}^{k}, w \in \mathbb{R}$. The image $T_{R, r}^{j, k}$ is the tube of radius $r$ about $S^{j}(R) \subset \mathbb{R}^{j+1} \times\{0\}$. Note that $j$ and $k$ are not treated symmetrically: for $j \neq k$ the two embeddings $T_{R, r}^{j, k}$ and $T_{R, r}^{k, j}$ of $S^{j} \times S^{k}$ into $\mathbb{R}^{j+k+1}$ are distinct (even disregarding the interchange of the factors).

Next let $r_{1}^{2}+r_{2}^{2}=1$ and recall the product embedding $S^{j}\left(r_{1}\right) \times S^{k}\left(r_{2}\right) \subset S^{j+k+1} \subset$ $\mathbb{R}^{j+1} \times \mathbb{R}^{k+1}$. Consider its image under stereographic projection, where the base point for the stereographic projection is a point of $S^{j+k+1}$ lying in $\{0\} \times \mathbb{R}^{k+1}$, which we can take to be $(0, \ldots, 0,1)$ by a rotation of $\mathbb{R}^{k+1}$. Thus the stereographic projection $\pi: S^{j+k+1} \rightarrow \mathbb{R}^{j+k+1}$ is

$$
\pi\left(x^{\prime}, x_{j+k+2}\right)=\frac{x^{\prime}}{1-x_{j+k+2}}, \quad \quad x^{\prime}=\left(x_{1}, \ldots, x_{j+k+1}\right) .
$$

It is an easy verification which we leave to the reader that $\pi\left(S^{j}\left(r_{1}\right) \times S^{k}\left(r_{2}\right)\right)=T_{R, r}^{j, k}$ with $R=1 / r_{1}$ and $r=r_{2} / r_{1}$. Since $\pi$ is conformal, $\mathcal{E}\left(S^{j}\left(r_{1}\right) \times S^{k}\left(r_{2}\right)\right)=\mathcal{E}\left(T_{R, r}^{j, k}\right)$. Thus the $\mathcal{E}$-critical embeddings of $S^{2} \times S^{2}$ and $S^{1} \times S^{3}$ in $S^{5}$ discussed above give $\mathcal{E}$-critical "anchor ring" tubes in $\mathbb{R}^{5}$. For $S^{2} \times S^{2}$, we obtain the single critical anchor ring tube $T_{\sqrt{2}, 1}^{2,2}$, which is the stereographic image of the minimal $S^{2}\left(\frac{1}{\sqrt{2}}\right) \times S^{2}\left(\frac{1}{\sqrt{2}}\right) \subset S^{5}$. For $S^{1} \times S^{3}$, we obtain the two critical anchor ring tubes $T_{2, \sqrt{3}}^{1,3}$ and $T_{2 / \sqrt{3}, 1 / \sqrt{3}}^{3,1}$ corresponding to the minimal embedding, and $T_{\sqrt{8 / 3}, \sqrt{5 / 3}}^{1,3}$ and $T_{\sqrt{8 / 5}, \sqrt{3 / 5}}^{3,1}$ corresponding to the non-minimal embedding. Note that $T_{R, r}^{j, k}$ is a dilate of $T_{c R, c r}^{j, k}$ for any $c>0$, so we conclude from the last three embeddings that $T_{2,1}^{3,1}, T_{\sqrt{8}, \sqrt{5}}^{1,3}$, and $T_{\sqrt{8}, \sqrt{3}}^{3,1}$ are $\mathcal{E}$-critical.

We have written down generalizations of the above embeddings to "generalized anchor rings" which arise as the images under stereographic projection of $S^{j_{1}}\left(r_{1}\right) \times \cdots S^{j_{l}}\left(r_{l}\right) \subset$ $S^{j_{1}+\ldots+j_{l}+l-1} \subset \mathbb{R}^{j_{1}+\ldots+j_{l}+l}$ for $\sum_{i=1}^{l} r_{i}^{2}=1$. Specializing to the $\mathcal{E}$-critical products $S^{1}\left(r_{1}\right) \times$ $S^{1}\left(r_{2}\right) \times S^{2}\left(r_{3}\right) \subset S^{6}$ and $S^{1}\left(r_{1}\right) \times S^{1}\left(r_{2}\right) \times S^{1}\left(r_{3}\right) \times S^{1}\left(r_{4}\right) \subset S^{7}$ discussed above gives $\mathcal{E}$-critical generalized anchor ring embeddings in $\mathbb{R}^{6}$ and $\mathbb{R}^{7}$.

We next exhibit a family of embeddings of $S^{2} \times S^{2}$ in $\mathbb{R}^{5}$ with energy unbounded above. For $a>0$, let $\delta_{a}: \mathbb{R}^{5} \rightarrow \mathbb{R}^{5}$ be given by $\delta_{a}(y, v)=(y, a v)$ for $y \in \mathbb{R}^{3}, v \in \mathbb{R}^{2}$.

\section{Proposition 6.2.}

as $a \rightarrow \infty$.

$$
\mathcal{E}\left(\delta_{a}\left(T_{\sqrt{2}, 1}^{2,2}\right)\right)=\frac{2 \pi^{2}}{35} a^{4}+o\left(a^{4}\right)
$$

Proof. Conformal invariance implies $\mathcal{E}\left(\delta_{a}\left(T_{\sqrt{2}, 1}\right)\right)=\mathcal{E}\left(\delta_{a}\left(T_{1,1 / \sqrt{2}}\right)\right)$. (We suppress the ${ }^{2,2}$ on $T_{R, r}^{2,2}$ throughout this proof.) Consider $\delta_{a}\left(T_{1, r}\right)$ for $0<r<1$; we will set $r=1 / \sqrt{2}$ later.

Parametrize $\delta_{a}\left(T_{1, r}\right)$ by introducing spherical coordinates for $y, z$ in (6.8):

$$
x=\left(\left(1+r \cos \phi_{2}\right) y, a r v\right)
$$


with

$$
\begin{aligned}
& y=\left(\sin \phi_{1} \cos \theta_{1}, \sin \phi_{1} \sin \theta_{1}, \cos \phi_{1}\right) \\
& v=\sin \phi_{2}\left(\cos \theta_{2}, \sin \theta_{2}\right),
\end{aligned}
$$

where $0 \leq \theta_{1}, \theta_{2}<2 \pi, 0 \leq \phi_{1}, \phi_{2} \leq \pi$. The tangent vectors $e_{1}=x_{\phi_{1}}, e_{2}=x_{\theta_{1}}, e_{3}=x_{\phi_{2}}$, $e_{4}=x_{\theta_{2}}$ are orthogonal with

$$
\begin{aligned}
& \left|x_{\phi_{1}}\right|^{2}=\left(1+r \cos \phi_{2}\right)^{2} \\
& \left|x_{\theta_{1}}\right|^{2}=\left(1+r \cos \phi_{2}\right)^{2} \sin ^{2} \phi_{1} \\
& \left|x_{\phi_{2}}\right|^{2}=r^{2}\left(a^{2} \cos ^{2} \phi_{2}+\sin ^{2} \phi_{2}\right) \\
& \left|x_{\theta_{2}}\right|^{2}=a^{2} r^{2} \sin ^{2} \phi_{2} .
\end{aligned}
$$

A unit normal is

$$
\nu=\frac{1}{\ell\left(\phi_{2}\right)}\left(a\left(\cos \phi_{2}\right) y, v\right)
$$

where $\ell\left(\phi_{2}\right)=\sqrt{a^{2} \cos ^{2} \phi_{2}+\sin ^{2} \phi_{2}}=\sqrt{1+\left(a^{2}-1\right) \cos ^{2} \phi_{2}}$. The second fundamental form (6.5) in this basis is

$$
L_{\alpha \beta}=-\frac{a}{\ell\left(\phi_{2}\right)}\left(\begin{array}{cccc}
\cos \phi_{2}\left(1+r \cos \phi_{2}\right) & 0 & 0 & 0 \\
0 & \cos \phi_{2}\left(1+r \cos \phi_{2}\right) \sin ^{2} \phi_{1} & 0 & 0 \\
0 & 0 & r & 0 \\
0 & 0 & 0 & r \sin ^{2} \phi_{2}
\end{array}\right)
$$

Recalling (6.9), contraction gives:

$$
\begin{aligned}
H & =-\frac{a}{\ell\left(\phi_{2}\right)}\left[\frac{2 \cos \phi_{2}}{1+r \cos \phi_{2}}+\frac{1}{r \ell\left(\phi_{2}\right)^{2}}+\frac{1}{r a^{2}}\right] \\
|L|^{2} & =\frac{a^{2}}{\ell\left(\phi_{2}\right)^{2}}\left[\frac{2 \cos ^{2} \phi_{2}}{\left(1+r \cos \phi_{2}\right)^{2}}+\frac{1}{r^{2} \ell\left(\phi_{2}\right)^{4}}+\frac{1}{r^{2} a^{4}}\right] .
\end{aligned}
$$

Since $H$ depends only on $\phi_{2}$, it follows that

$$
|\nabla H|^{2}=r^{-2} \ell\left(\phi_{2}\right)^{-2}\left(\partial_{\phi_{2}} H\right)^{2} .
$$

This information is sufficient to calculate the integrand $|\nabla H|^{2}-|L|^{2} H^{2}+\frac{7}{16} H^{4}$ of $\underline{\mathcal{E}}\left(\delta_{a}\left(T_{1, r}\right)\right)$, and clearly it depends only on $\phi_{2}$. Now (6.9) also gives

$$
d a=a r^{2}\left(1+r \cos \phi_{2}\right)^{2} \ell\left(\phi_{2}\right) \sin \phi_{2} \sin \phi_{1} d \phi_{1} d \theta_{1} d \phi_{2} d \theta_{2}
$$

It follows that we can write

$$
\left(|\nabla H|^{2}-|L|^{2} H^{2}+\frac{7}{16} H^{4}\right) d a=I\left(\cos \phi_{2}\right) \sin \phi_{1} \sin \phi_{2} d \phi_{1} d \theta_{1} d \phi_{2} d \theta_{2}
$$

where $I\left(\cos \phi_{2}\right)$ is a function only of $\cos \phi_{2}$ (depending on $a$ and $r$ ). Upon making the substitution $s=\cos \phi_{2}$, it follows that

$$
\underline{\mathcal{E}}\left(\delta_{a}\left(T_{1, r}\right)\right)=8 \pi^{2} \int_{-1}^{1} I(s) d s .
$$


HIGHER-DIMENSIONAL WILLMORE ENERGIES VIA MINIMAL SUBMANIFOLD ASYMPTOTICS 35 We used Mathematica to calculate $I(s)$ for $r=1 / \sqrt{2}$. The result is

$$
I(s)=-\frac{1}{8 a^{3}} \frac{p(a, s)}{\left(1+\frac{s}{\sqrt{2}}\right)^{2}\left(1+\left(a^{2}-1\right) s^{2}\right)^{11 / 2}},
$$

where $p(a, s)$ is the polynomial in $a, s$ given by:

$$
\begin{aligned}
& p(a, s)=4 s^{12} a^{16} \\
& +\left(-24 s^{12}-24 \sqrt{2} s^{11}-24 s^{10}-24 \sqrt{2} s^{9}\right) a^{14} \\
& +\left(54 s^{12}+92 \sqrt{2} s^{11}-16 s^{10}-128 \sqrt{2} s^{9}-218 s^{8}-172 \sqrt{2} s^{7}-132 s^{6}\right) a^{12} \\
& +\left(-50 s^{12}-106 \sqrt{2} s^{11}+226 s^{10}+502 \sqrt{2} s^{9}+246 s^{8}-162 \sqrt{2} s^{7}-234 s^{6}-286 \sqrt{2} s^{5}\right. \\
& \left.-380 s^{4}-188 \sqrt{2} s^{3}-144 s^{2}\right) a^{10} \\
& +\left(\frac{9 s^{12}}{4}-7 \sqrt{2} s^{11}-354 s^{10}-440 \sqrt{2} s^{9}+\frac{541 s^{8}}{2}+632 \sqrt{2} s^{7}+25 s^{6}-350 \sqrt{2} s^{5}\right. \\
& \left.-\frac{847 s^{4}}{4}+95 \sqrt{2} s^{3}+327 s^{2}+118 \sqrt{2} s+9\right) a^{8} \\
& +\left(27 s^{12}+88 \sqrt{2} s^{11}+208 s^{10}-34 \sqrt{2} s^{9}-478 s^{8}-42 \sqrt{2} s^{7}+816 s^{6}+398 \sqrt{2} s^{5}\right. \\
& \left.-241 s^{4}-302 \sqrt{2} s^{3}-304 s^{2}-108 \sqrt{2} s-28\right) a^{6} \\
& +\left(-\frac{25 s^{12}}{2}-38 \sqrt{2} s^{11}-16 s^{10}+146 \sqrt{2} s^{9}+165 s^{8}-322 \sqrt{2} s^{7}-570 s^{6}+254 \sqrt{2} s^{5}\right. \\
& \left.+\frac{1523 s^{4}}{2}+64 \sqrt{2} s^{3}-318 s^{2}-104 \sqrt{2} s-10\right) a^{4} \\
& +\left(-3 s^{12}-14 \sqrt{2} s^{11}-42 s^{10}-4 \sqrt{2} s^{9}+100 s^{8}+84 \sqrt{2} s^{7}-22 s^{6}-88 \sqrt{2} s^{5}\right. \\
& \left.-69 s^{4}+10 \sqrt{2} s^{3}+32 s^{2}+12 \sqrt{2} s+4\right) a^{2} \\
& +\left(\frac{9 s^{12}}{4}+9 \sqrt{2} s^{11}+18 s^{10}-18 \sqrt{2} s^{9}-\frac{171 s^{8}}{2}-18 \sqrt{2} s^{7}+117 s^{6}+72 \sqrt{2} s^{5}\right. \\
& \left.-\frac{207 s^{4}}{4}-63 \sqrt{2} s^{3}-9 s^{2}+18 \sqrt{2} s+9\right) .
\end{aligned}
$$

Observe that $p(a, s)$ has degree 16 in $a$ and any monomial $s^{i} a^{j}$ occurring in $p$ has $j-i \leq 8$. Moreover, the only terms with $j-i=8$ are $9 a^{8}-144 s^{2} a^{10}$.

In order to derive the asymptotics of $\underline{\mathcal{E}}\left(\delta_{a}\left(T_{1,1 / \sqrt{2}}\right)\right)$ as $a \rightarrow \infty$, we have the following lemma. Set

$$
\mathcal{I}_{i, j}(a)=\int_{-1}^{1} \frac{a^{j} s^{i} d s}{\left(1+\frac{s}{\sqrt{2}}\right)^{2}\left(1+\left(a^{2}-1\right) s^{2}\right)^{11 / 2}}
$$


Lemma 6.3.

$$
\lim _{a \rightarrow \infty} \mathcal{I}_{i, j}(a)= \begin{cases}\int_{-\infty}^{\infty} \frac{t^{i} d t}{\left(t^{2}+1\right)^{11 / 2}} & j-i=1, i<10 \\ 0 & j-i<1, j<11\end{cases}
$$

Proof. Make the substitution $t=a s$ to rewrite

$$
\mathcal{I}_{i, j}(a)=a^{j-i-1} \int_{-a}^{a} \frac{t^{i} d t}{\left(1+\frac{t}{a \sqrt{2}}\right)^{2}\left(1+t^{2}-(t / a)^{2}\right)^{11 / 2}} .
$$

If $j-i=1$ and $i<10$, dominated convergence shows that $\lim _{a \rightarrow \infty} \mathcal{I}_{i, j}(a)=\int_{-\infty}^{\infty} \frac{t^{i} d t}{\left(t^{2}+1\right)^{11 / 2}}$. If $j-i<1$ and $i<10$, the same argument shows that the limit is 0 . If $j-i<1, j<11$, and $i \geq 10$, choose $\epsilon$ with $0<\epsilon<11-j$. Then

$$
\left|\int_{-a}^{a} \frac{a^{j-i-1} t^{i} d t}{\left(1+\frac{t}{a \sqrt{2}}\right)^{2}\left(1+t^{2}-(t / a)^{2}\right)^{11 / 2}}\right| \leq \int_{-a}^{a} \frac{a^{j-i-1}|t|^{10-\epsilon}|t|^{i+\epsilon-10} d t}{\left(1+\frac{t}{a \sqrt{2}}\right)^{2}\left(1+t^{2}-(t / a)^{2}\right)^{11 / 2}} .
$$

Since $|t| \leq a$ and $i+\epsilon \geq 10$, we have

$$
a^{j-i-1}|t|^{10-\epsilon}|t|^{i+\epsilon-10} \leq a^{j-i-1}|t|^{10-\epsilon} a^{i+\epsilon-10}=a^{j+\epsilon-11}|t|^{10-\epsilon} .
$$

Thus dominated convergence again shows that the limit is 0 .

According to (6.10), (6.11), (6.12), $a^{-4} \underline{\mathcal{E}}\left(\delta_{a}\left(T_{1,1 / \sqrt{2}}\right)\right)$ is a linear combination of integrals of the form $\mathcal{I}_{i, j}(a)$ with $j \leq 9$ and $j-i \leq 1$. Lemma 6.3 shows that the limit vanishes for all terms with $j-i<1$. As indicated above, this is all but two terms. Thus

$$
\lim _{a \rightarrow \infty} a^{-4} \underline{\mathcal{E}}\left(\delta_{a}\left(T_{1,1 / \sqrt{2}}\right)\right)=-\pi^{2} \lim _{a \rightarrow \infty}\left(9 \mathcal{I}_{0,1}(a)-144 \mathcal{I}_{2,3}(a)\right)=\frac{256 \pi^{2}}{35} .
$$

The result follows upon recalling that $\underline{\mathcal{E}}=128 \mathcal{E}$.

Proof of Proposition 1.1. It was shown in $₫ 6.1$ that in the three cases other than $S^{2} \times S^{2} \subset S^{5}$, $\mathcal{E}$ is already unbounded above and below when restricted to the families considered there. It was also shown in $\$ 6.1$ that $\mathcal{E}$ is unbounded below when restricted to the corresponding family for $S^{2} \times S^{2} \subset S^{5}$. Proposition 6.2 shows that $\mathcal{E}$ is unbounded above over embeddings $S^{2} \times S^{2} \subset S^{5}$.

6.3. Second Variation in $S^{5}$. In this section we calculate the second variation of $\mathcal{E}$ at a minimal immersed hypersurface $\Sigma$ in $S^{5}$. We specialize the general formula to $\Sigma=S^{4}$ and $\Sigma=S^{2}(1 / \sqrt{2}) \times S^{2}(1 / \sqrt{2})$.

Let $f: \Sigma \rightarrow S^{5}$ be a minimal immersion of $\Sigma^{4}$ in $S^{5}$, and $F: \Sigma \times(-\epsilon, \epsilon)$ be a variation of $f$, i.e. $F_{0}=f$. Let $V=\left.\partial_{t} F_{t}\right|_{t=0}$ denote the variational vector field. We assume throughout that $V$ is normal. Recall the first and second variations of area:

$$
\begin{aligned}
\left.\partial_{t} A\left(F_{t}(\Sigma)\right)\right|_{t=0} & =-\int_{\Sigma}\langle H, V\rangle d a_{\Sigma} \\
\left.\nabla_{\partial_{t}} H\right|_{t=0} & =-J V \\
\left.\partial_{t}^{2} A\left(F_{t}(\Sigma)\right)\right|_{t=0} & =\int_{\Sigma}\langle J V, V\rangle d a_{\Sigma} .
\end{aligned}
$$


Here $\nabla_{\partial_{t}}$ refers to the pullback connection on the pullback of the normal bundle and $J=$ $-\Delta-4-|L|^{2}$ is the Jacobi operator, where $\Delta$ denotes the normal bundle Laplacian.

Proposition 6.4. If $\Sigma$ is a minimal immersed hypersurface in $S^{5}$, then

$$
\left.\partial_{t}^{2} \underline{\mathcal{E}}\left(F_{t}(\Sigma)\right)\right|_{t=0}=\int_{\Sigma}\langle\mathcal{J} V, V\rangle d a_{\Sigma}
$$

where

$$
\mathcal{J}=2 J(J+4)(J+6) \text {. }
$$

Proof. Recall that $\mathcal{E}$ is given by $(1.2)$ and $\underline{\mathcal{E}}=128 \mathcal{E}$. Since $\left.H\right|_{t=0}=0$, the $|H|^{4}$ term does not contribute to the second variation. Since $|\nabla H|^{2}-|L|^{2}|H|^{2}+6|H|^{2}$ vanishes to second order at $t=0$, we have

$$
\left.\partial_{t}^{2} \underline{\mathcal{E}}\left(F_{t}(\Sigma)\right)\right|_{t=0}=\left.\int_{\Sigma} \partial_{t}^{2}\right|_{t=0}\left(|\nabla H|^{2}-|L|^{2}|H|^{2}+6|H|^{2}\right) d a_{\Sigma}+48 \int_{\Sigma}\langle J V, V\rangle d a_{\Sigma}
$$

Again using that $\left.H\right|_{t=0}=0$, we have

$$
\begin{aligned}
\left.\partial_{t}^{2}\right|_{t=0}\left(|\nabla H|^{2}-|L|^{2}|H|^{2}+6|H|^{2}\right) & =2\left|\nabla \nabla_{\partial_{t}} H\right|^{2}-2|L|^{2}\left|\nabla_{\partial_{t}} H\right|^{2}+12\left|\nabla_{\partial_{t}} H\right|^{2} \\
& =2\left(|\nabla J V|^{2}-|L|^{2}|J V|^{2}+6|J V|^{2}\right) .
\end{aligned}
$$

So

$$
\begin{aligned}
\left.\partial_{t}^{2} \underline{\mathcal{E}}\left(F_{t}(\Sigma)\right)\right|_{t=0} & =2 \int_{\Sigma}\left\langle-\Delta J V-|L|^{2} J V+6 J V+24 V, J V\right\rangle d a_{\Sigma} \\
& =2 \int_{\Sigma}\langle(J+4) J V+6 J V+24 V, J V\rangle d a_{\Sigma} \\
& =2 \int_{\Sigma}\langle J(J+4)(J+6) V, V\rangle d a_{\Sigma} .
\end{aligned}
$$

Proposition 6.4 shows that the second variation of $\underline{\mathcal{E}}$ is determined by the spectral decomposition of the self-adjoint Jacobi operator $J$. We identify this for $\Sigma=S^{4}$ and $\Sigma=$ $S^{2}(1 / \sqrt{2}) \times S^{2}(1 / \sqrt{2})$. The subsequent argument follows closely that of [W], where the second variation of the classical Willmore energy at a minimal surface is identified. We refer to W] for elaboration and proofs of some of the statements which follow.

Let $\mathcal{K}$ denote the 15-dimensional space of Killing fields of $S^{5}$ and let $\mathcal{K}_{\Sigma} \subset \Gamma(N \Sigma)$ denote the space of normal projections of restrictions to $\Sigma$ of elements of $\mathcal{K}$. The kernel of the restriction-projection map $\mathcal{K} \rightarrow \mathcal{K}_{\Sigma}$ is the space of Killing fields whose restriction to $\Sigma$ is everywhere tangent to $\Sigma$. Its dimension equals the dimension of the space of isometries of $S^{5}$ which map $\Sigma$ to itself. For $\Sigma=S^{4}$ this dimension is 10 , while for $\Sigma=S^{2}(1 / \sqrt{2}) \times S^{2}(1 / \sqrt{2})$ it is 6 . So $\operatorname{dim} \mathcal{K}_{S^{4}}=5$ and $\operatorname{dim} \mathcal{K}_{S^{2}(1 / \sqrt{2}) \times S^{2}(1 / \sqrt{2})}=9$. For any $\Sigma, \mathcal{K}_{\Sigma} \subset \operatorname{ker} J$.

Let $\mathcal{C}$ denote the 6 -dimensional space of tangential projections of restrictions to $S^{5}$ of constant vector fields on $\mathbb{R}^{6}$, and let $\mathcal{C}_{\Sigma} \subset \Gamma(N \Sigma)$ denote the space of normal projections of restrictions to $\Sigma$ of elements of $\mathcal{C}$. Every element of $\mathcal{C}$ is a conformal Killing field of $S^{5}$ and the space of conformal Killing fields of $S^{5}$ equals $\mathcal{K} \oplus \mathcal{C}$. The dimension of $\mathcal{C}_{\Sigma}$ is 1 if $\Sigma$ is a totally geodesic $S^{4} \subset S^{5}$, and is 6 otherwise. For any $\Sigma, \mathcal{C}_{\Sigma} \subset \operatorname{ker}(J+4)$. 
The space of conformal directions to $\Sigma$ is $\mathcal{K}_{\Sigma} \oplus \mathcal{C}_{\Sigma} \subset \Gamma(N \Sigma)$. These are in the kernel of $\mathcal{J}$ by conformal invariance; this is consistent with (6.13) and the facts that $\mathcal{K}_{\Sigma} \subset$ ker $J$, $\mathcal{C}_{\Sigma} \subset \operatorname{ker}(J+4)$.

Proof of Proposition 1.2. The normal bundle to $S^{4} \subset S^{5}$ has a parallel nonvanishing section. So its space of sections can be identified with $C^{\infty}\left(S^{4}\right)$ and the normal bundle Laplacian with the scalar Laplacian. The eigenvalues of $-\Delta$ are $j(j+3)=0,4,10, \cdots$ with multiplicities $\left(\begin{array}{c}j+4 \\ 4\end{array}\right)-\left(\begin{array}{c}j+2 \\ 4\end{array}\right)=1,5,14, \cdots$. So the eigenvalues of $J=-\Delta-4$ are $-4,0,6, \cdots$ with the same multiplicities. By comparing dimensions we see that $\operatorname{ker} J=\mathcal{K}_{S^{4}}$ and $\operatorname{ker}(J+4)=\mathcal{C}_{S^{4}}$. So the kernel of $\mathcal{J}$ is exactly the conformal directions. Since all other eigenvalues of $J$ are positive, we conclude that $\mathcal{J}$ is positive transverse to the conformal directions.

Proposition 6.5. The second variation of $\mathcal{E}$ at $S^{2}(1 / \sqrt{2}) \times S^{2}(1 / \sqrt{2}) \subset S^{5}$ has one negative eigendirection, the direction of the family $S^{2}\left(r_{1}\right) \times S^{2}\left(r_{2}\right)$ considered in 86.1 . It is positive in all eigendirections transverse to this direction and to the tangent space to the orbit of the conformal group.

Proof. The normal bundle to $\Sigma=S^{2}(1 / \sqrt{2}) \times S^{2}(1 / \sqrt{2}) \subset S^{5}$ has a parallel nonvanishing section given by (6.4) with $r_{1}=r_{2}$. So its Laplacian can be identified with two copies of the scalar Laplacian of $S^{2}(1 / \sqrt{2})$. The eigenvalues of $-\Delta_{S^{2}(1 / \sqrt{2})}$ are $2 j(j+1)=0,4,12, \cdots$ with multiplicities $\left(\begin{array}{c}j+2 \\ 2\end{array}\right)-\left(\begin{array}{c}j \\ 2\end{array}\right)=2 j+1=1,3,5, \cdots$. So the eigenvalues of $-\Delta_{\Sigma}$ are $0,4,8,12, \cdots$ with multiplicities $1,6,9,10, \cdots$. We have $|L|^{2}=4$ from (6.6), so $J=-\Delta-8$. Hence the eigenvalues of $J$ are $-8,-4,0,4, \cdots$ with multiplicities $1,6,9,10, \cdots$. Comparing dimensions shows that $\operatorname{ker} J=\mathcal{K}_{\Sigma}$ and $\operatorname{ker}(J+4)=\mathcal{C}_{\Sigma}$. Thus $\mathcal{J}$ has exactly one negative direction, its kernel consists precisely of the conformal directions, and there is a complementary space to these on which $\mathcal{J}$ is positive. The -8 eigenspace of $J$ is spanned by constant multiples of $\nu$ from (6.4). This is the variation field of the family $S^{2}\left(r_{1}\right) \times S^{2}\left(r_{2}\right)$ analyzed in 6.1 . We noted there that the family had a local maximum at $r_{1}=r_{2}=1 / \sqrt{2}$ and it is easily seen from (6.7) that the second derivative at this maximum is negative. So for $S^{2}(1 / \sqrt{2}) \times S^{2}(1 / \sqrt{2})$, this is the only eigendirection in which $\mathcal{E}$ decreases.

6.4. Other Energies. Other conformally invariant energies of $\Sigma^{4} \subset M^{n}$ can be constructed by adding to $\mathcal{E}$ conformally invariant expressions. The trace-free part $\stackrel{\circ}{L}$ scales upon conformal transformation of the metric, so $\int_{\Sigma} p(\stackrel{L}{L}) d a_{\Sigma}$ is conformally invariant for any quartic scalar contraction $p(\stackrel{\circ}{L})$ of the trace-free second fundamental form. The following proposition shows that upon adding appropriate multiples of $|\stackrel{\circ}{L}|^{4}$, one obtains non-negative energies. Recall that $\underline{\mathcal{E}}=128 \mathcal{E}$ with $\mathcal{E}$ given by (1.1) when $M$ is a Euclidean space.

Proposition 6.6. Suppose $(M, g)$ is $\mathbb{R}^{n}$ with the Euclidean metric. If $\beta \geq \frac{4}{3}$, then the energy

$$
\underline{\mathcal{E}}+\beta \int_{\Sigma}|\stackrel{\circ}{L}|^{4} d a_{\Sigma}
$$

is non-negative.

Proof. Decomposing $L_{\alpha \beta}^{\alpha^{\prime}}=\stackrel{\circ}{L}_{\alpha \beta}^{\alpha^{\prime}}+\frac{1}{4} H^{\alpha^{\prime}} g_{\alpha \beta}$ gives

$$
\underline{\mathcal{E}}=\int_{\Sigma}\left(|\nabla H|^{2}-\left|\stackrel{\circ}{L}^{t} H\right|^{2}+\frac{3}{16}|H|^{4}\right) d a_{\Sigma}
$$


Now $\left.\beta\left|\stackrel{\circ}{L}^{4}-\right| \stackrel{\circ}{L}^{t} H\right|^{2}+\frac{3}{16}|H|^{4} \geq 0$ since the quadratic form $\beta x^{2}-x y+\frac{3}{16} y^{2}$ is nonnegative for $\beta \geq \frac{4}{3}$.

For the subsequent discussion, we denote by $\bar{W}$ and $\bar{P}$ the Weyl and Schouten tensors of the induced metric on $\Sigma$. If $\operatorname{dim} \Sigma=4$, then $\int_{\Sigma}|\bar{W}|^{2} d a_{\Sigma}$ is conformally invariant. The Chern-Gauss-Bonnet formula in dimension 4 states

$$
32 \pi^{2} \chi(\Sigma)=\int_{\Sigma}\left[|\bar{W}|^{2}+16 \sigma_{2}(\bar{P})\right] d a_{\Sigma}
$$

where $\sigma_{2}(\bar{P})=\frac{1}{2}\left((\operatorname{tr} \bar{P})^{2}-|\bar{P}|^{2}\right)$ is the second elementary symmetric function of the eigenvalues of the Schouten tensor. In particular, $\int_{\Sigma} \sigma_{2}(\bar{P}) d a_{\Sigma}$ is also conformally invariant. So further conformally invariant energies can be obtained by adding a linear combination of the integrals of $|\bar{W}|^{2}$ and $\sigma_{2}(\bar{P})$. The Gauss equation can be used to express $\bar{W}$ and $\bar{P}$ in terms of $L$ and curvature of $g$.

In [V], Vyatkin has used tractor calculus to derive a conformally invariant energy for 4dimensional hypersurfaces in conformally flat 5-manifolds. By Theorem 5.2.4 and Lemma 5.2.7 of [V], his energy takes the form $\mathcal{V}=\int_{\Sigma} V d a_{\Sigma}$, where

$$
V=-\frac{2}{3} \stackrel{\circ}{L}^{\alpha \beta} \nabla_{\alpha} \nabla^{\gamma} \stackrel{\circ}{L}_{\gamma \beta}-4 \bar{P}_{\alpha}^{\gamma} \stackrel{\circ}{L}^{\alpha \beta} \stackrel{\circ}{L}_{\gamma \beta}+2 \bar{P}_{\alpha}{ }^{\alpha}|\stackrel{\circ}{L}|^{2}-\frac{2}{9} \nabla^{\beta} \stackrel{\circ}{L}_{\beta}{ }^{\alpha} \nabla^{\gamma} \stackrel{\circ}{L}_{\alpha \gamma} .
$$

Vyatkin's energy can be related to $\mathcal{E}$ in the case that $M$ is 5 -dimensional Euclidean space as follows. The contracted Codazzi-Mainardi equation gives $\nabla^{\alpha} \stackrel{\circ}{L}_{\alpha \beta}=\frac{3}{4} \nabla_{\beta} H$. Integrating by parts one of the derivatives in the first term and then combining the first and last terms shows that

$$
\mathcal{V}=\int_{\Sigma}\left(\frac{1}{4}|\nabla H|^{2}-4 \bar{P}_{\alpha}^{\gamma} \stackrel{\circ}{L}^{\alpha \beta} \stackrel{\circ}{L}_{\gamma \beta}+2 \bar{P}_{\alpha}^{\alpha}|\stackrel{\circ}{L}|^{2}\right) d a_{\Sigma}
$$

The intrinsic Schouten tensor $\bar{P}$ can be expressed as a linear combination of quadratic terms in $L$ via the Gauss equation. Doing so, substituting and simplifying gives

$$
-4 \bar{P}_{\alpha}{ }^{\gamma} \stackrel{\circ}{L}^{\alpha \beta} \stackrel{\circ}{L}_{\gamma \beta}+2 \bar{P}_{\alpha}{ }^{\alpha}|\stackrel{\circ}{L}|^{2}=2 \operatorname{tr} \stackrel{\circ}{L}^{4}-\frac{2}{3}|\stackrel{\circ}{L}|^{4}-H \operatorname{tr} \stackrel{\circ}{L}^{3}+\frac{1}{8} H^{2}|\stackrel{\circ}{L}|^{2} .
$$

Similar calculations express $|\bar{W}|^{2}$ and $\sigma_{2}(\bar{P})$ in terms of $L$ :

$$
\begin{gathered}
|\bar{W}|^{2}=\frac{7}{3}|\stackrel{\circ}{L}|^{4}-4 \operatorname{tr} \stackrel{\circ}{L}^{4} \\
4 \sigma_{2}(\bar{P})=\operatorname{tr} \stackrel{\circ}{L}^{4}-\frac{1}{3}|\stackrel{\circ}{L}|^{4}-H \operatorname{tr} \stackrel{\circ}{L}^{3}+\frac{3}{8} H^{2}|\stackrel{\circ}{L}|^{2}-\frac{3}{64} H^{4} .
\end{gathered}
$$

Combining (6.14), (6.15), (6.16), (6.17), and (6.18) gives

$$
\mathcal{V}=\frac{1}{4} \underline{\mathcal{E}}+8 \pi^{2} \chi(\Sigma)+\int_{\Sigma}\left(2 \operatorname{tr} \stackrel{\circ}{L}^{4}-\frac{11}{12}|\stackrel{\circ}{L}|^{4}\right) d a_{\Sigma}
$$

\section{REFERENCES}

[A1] M. Anderson, Complete minimal varieties in hyperbolic space, Invent. Math. 69 (1982), $477-494$.

[A2] M. Anderson, Complete minimal hypersurfaces in hyperbolic n-manifolds, Comment. Math. Helv. 58 (1983), 264-290.

[HSS] S. de Haro, K. Skenderis and S.N. Solodukhin, Holographic reconstruction of spacetime and renormalization in the AdS/CFT correspondence, Comm. Math. Phys. 217 (2001), 594-622, arXiv:hep-th/0002230

[FG] C. Fefferman and C. R. Graham, The Ambient Metric, Princeton University Press, 2012, arXiv:math/0710.0919. 
[GGHW] M. Glaros, A. R. Gover, M. Halbasch and A. Waldron, Singular Yamabe problem Willmore energies, arXiv:1508.01838.

[GoW1] A. R. Gover and A. Waldron, Boundary calculus for conformally compact manifolds, Indiana Univ. Math. J. 63 (2014), 120-163, arXiv:1104.2991.

[GoW2] A. R. Gover and A. Waldron, Generalising the Willmore equation: submanifold conformal invariants from a boundary Yamabe problem, arXiv:1407.6742.

[GoW3] A. R. Gover and A. Waldron, Conformal hypersurface geometry via a boundary Loewner-NirenbergYamabe problem, arXiv:1506.02723.

[GoW4] A. R. Gover and A. Waldron, Renormalized volume, arXiv:1603.07367.

[GoW5] A. R. Gover and A. Waldron, A calculus for conformal hypersurfaces and new higher Willmore energy functionals, arXiv: 1611.04055

[Gr1] C. R. Graham, Volume and area renormalizations for conformally compact Einstein metrics, Rend. Circ. Mat. Palermo, Ser. II, Suppl. 63 (2000), 31-42, arXiv:math/9909042.

[Gr2] C. R. Graham, Volume renormalization for singular Yamabe metrics, Proc. Amer. Math. Soc., 145 (2017), 1781-1792, arXiv:1606.00069.

[GH] C. R. Graham and K. Hirachi, The ambient obstruction tensor and Q-curvature, in AdS/CFT Correspondence: Einstein Metrics and their Conformal Boundaries, IRMA Lectures in Mathematics and Theoretical Physics 8 (2005), 59-71, arXiv:math/0405068.

[GW] C. R. Graham and E. Witten, Conformal anomaly of submanifold observables in AdS/CFT correspondence, Nuclear Physics B 546 (1999), 52-64, arXiv:hep-th/9901021.

[Gu1] J. Guven, Membrane geometry with auxiliary variables and quadratic constraints, J. Phys. A: Math. Gen. 37 (2004), L313-L319.

[Gu2] J. Guven, Conformally invariant bending energy for hypersurfaces, J. Phys. A: Math. Gen. 38 (2005), 7943-7955.

[HJ] Q. Han and X. Jiang, Boundary expansions for minimal graphs in the hyperbolic space, arXiv: 1412.7608.

[HSW] Q. Han, W. Shen and Y. Wang, Optimal regularity of minimal graphs in the hyperbolic space, Calc. Var. Par. Diff. Eq. 55 (2016), Art. 3, 19 pp., arXiv:1511.01143.

[HL] R. Hardt and F.-H. Lin, Regularity at infinity for area-minimizing hypersurfaces in hyperbolic space, Invent. Math. 88 (1987), 217-224.

[H] W. Y. Hsiang, On compact homogeneous minimal submanifolds, Proc. Nat. Acad. Sci. USA 56 (1966), 5-6.

[L1] F.-H. Lin, On the Dirichlet problem for minimal graphs in hyperbolic space, Invent. Math. 96 (1989), 593-612. Erratum: Invent. Math. 187 (2012), 755-757.

[L2] F.-H. Lin, Asymptotic behavior of area-minimizing currents in hyperbolic space, Comm. Pure Appl. Math. 42 (1989), 229-242.

[P] R. S. Palais, The principle of symmetric criticality, Comm. Math. Phys. 69 (1979), 19-30.

[T] Y. Tonegawa, Existence and regularity of constant mean curvature hypersurfaces in hyperbolic space, Math. Z. 221 (1996), 591-615.

[V] Y. Vyatkin, Manufacturing conformal invariants of hypersurfaces, Ph.D. thesis, University of Auckland (2013).

[W] J. L. Weiner, On a problem of Chen, Willmore, et al, Indiana Univ. Math. J. 27 (1978), 19-35.

[Z] Y. Zhang, Graham-Witten's conformal invariant for closed four dimensional submanifolds, arXiv:1703.08611.

Department of Mathematics, University of Washington, Box 354350, Seattle, WA 98195-4350, USA

E-mail address: robin@math.washington.edu

E-mail address: nwr@uw.edu 